Universidade de São Paulo

Instituto de Física

\title{
Estudo de modelos irreversíveis: processo de contato, pilha de areia assimétrico e Glauber linear
}

\author{
Evandro Freire da Silva
}

Tese de Doutorado apresentada ao Instituto de Física para a obtenção do título de Doutor em Ciências.

Orientador: Prof. Dr. Mário José de Oliveira

Comissão Examinadora:

Prof. Dr. Mário José de Oliveira — USP

Prof. Dr. André de Pinho Vieira - USP

Prof. Dr. Jurgen Fritz Stilck - UFF

Prof. Dr. José Guilherme Martins Alves Moreira - UFMG

Prof. Dr. Sílvio da Costa Ferreira Júnior - UFV

São Paulo 


\section{FICHA CATALOGRÁFICA}

Preparada pelo Serviço de Biblioteca e Informaçāo do Instituto de Física da Universidade de Sāo

\section{Paulo}

Silva, Evandro Freire da

Estudo de modelos irreversiveis : processo de contato, pilha de areia assimétrico e Glauber linear. São Paulo, 2009.

Tese (Doutorado) - Universidade de São Paulo. Instituto de Física, Departamento de Física Geral.

Orientad or: Prof. Dr. Mário José de Oliveir a

Área de Concentração: Física

Unitemos: 1. Mudança de fase; 2. Process os estocásticos; 3. Processo de contato; 4. Modelo de Ising.

USP/F/SBI-081/2009 
À memória de Manoel, meu pai. 



\section{Agradecimentos}

À minha mãe e aos meus irmãos, que, durante estes anos, têm oferecido suporte ao meu trabalho.

Ao professor Mário de Oliveira, que aceitou me orientar neste trabalho e dedicou parte de seu tempo a me auxiliar neste início de caminhada na Física.

Aos professores do Departamento de Física Geral, especialmente a Tânia Tomé, Vera Henriques e Sílvio Salinas, que me auxiliaram de diversas maneiras durante a minha Pós-Graduação.

Aos colegas e funcionários deste Instituto, especialmente a Henrique, Silas, Eduardo, Danilo, Masayuki, Hélder, Alexei, Áttila, David, Maicom, Paula B., Paula M., Fabiano, Marco Aurélio, Dirce, Silvana e Márcia, pela ajuda e pelo companheirismo. À FAPESP, pelo suporte financeiro.

E, acima de tudo, a Deus, sem O qual nada disso teria sido possível. 



\section{Resumo}

Neste trabalho estudamos alguns modelos estocásticos reversíveis e irreversíveis por meio de várias técnicas que incluem expansões em série, simulações numéricas e métodos analíticos. Primeiramente, construímos uma expansão supercrítica para a densidade do processo de contato em uma dimensão, que fornece a taxa crítica e o expoente crítico $\beta$ pelo método de aproximantes de Padé. Depois, examinamos um modelo de pilha de areia com restrição de altura assimétrico que apresenta fluxo de partículas não-nulo no estado estacionário e suas propriedades críticas são determinadas em função do parâmetro de assimetria $p$. Finalmente, estudamos de forma analítica o modelo de Glauber linear, que é idêntico, em uma dimensão, ao modelo de Glauber. Em qualquer número de dimensões, é possível obter uma expressão para a susceptibilidade do modelo de Glauber linear a partir da expansão em série perturbativa, cujos coeficientes são determinados em todas as ordens. Também discutimos como generalizar esse método para obter expansões em série para o modelo de Glauber em duas dimensões. 



\begin{abstract}
In this work we study some reversible and irreversible stochastic models using various techniques that include series expansions, numerical simulations and analytical methods. Firstly we write a supercritical series expansion of the particle density of the one-dimensional contact process, which gives us the critical annihilation rate and critical exponent $\beta$ after using the Padé approximants method. Secondly we examine the assimetric height restricted sandpile model, which presents a non-zero particle flux at the stationary active state and its critical properties are determined as a function of the assimetry parameter $p$. Finally we study analitically the linear Glauber model, which is identical in one dimension to the Glauber model. It is possible in any dimension to obtain an expression of the susceptibility of the linear Glauber model from a perturbative series expansion in which the coefficients can be determined at all orders. We also discuss how to generalize the method in order to obtain a series expansion for the Glauber model in two dimensions.
\end{abstract}





\section{Sumário}

1 Introdução 1

2 Processo de contato - expansão em série 5

2.1 O processo de contato . . . . . . . . . . . . . . . 5

2.2 Hierarquia de equações e expansão em série . . . . . . . . . . . . . 8

2.3 Análise de Padé . . . . . . . . . . . . . . . . . . . . . . . 13

2.4 Implementação do programa. Resultados . . . . . . . . . . . . . . 16

$\begin{array}{lll}3 & \text { O modelo de pilha de areia assimétrico } & 19\end{array}$

3.1 Criticalidade auto-organizada e modelos de pilha de areia . . . . . . . 19

3.2 Modelos de pilha de areia com restrição de altura . . . . . . . . . . . 20

3.3 O modelo PAA I - Generalidades . . . . . . . . . . . . . . . . . 22

3.3.1 Estudo do modelo para $p=0$ ou $1 \ldots \ldots$. . . . . . . 25

3.3.2 Fluxo de partículas . . . . . . . . . . . . . . . 29

3.4 O modelo PAA II - Aproximações de campo médio . . . . . . . . . . 31

3.4.1 Aproximação de campo médio simples . . . . . . . . . . . . . 32

3.4 .2 Aproximação de campo médio de pares . . . . . . . . . . . . . 34

3.4 .3 Aproximação de campo médio de trincas . . . . . . . . . . . . 40

3.4.4 Aproximações para o fluxo de partículas . . . . . . . . . . . 48

3.5 O modelo PAA III - Simulação . . . . . . . . . . . . . . . . . 52

3.5.1 Densidade crítica . . . . . . . . . . . . . . . 53

3.5.2 Fluxo de partículas . . . . . . . . . . . . . . 62

4 O modelo de Glauber linear $\quad 65$

4.1 O método de expansão em série perturbativa . . . . . . . . . . . . 67

4.1.1 Matriz de evolução . . . . . . . . . . . . . . . . . . . . . 67

4.1.2 Expansão em série perturbativa . . . . . . . . . . . . 70 
$4.2 \quad \mathrm{O}$ modelo GL unidimensional . . . . . . . . . . . . . . 71

4.2 .1 Aplicação do método de expansão em série . . . . . . . . . . 73

4.2 .2 Cálculo dos coeficientes $A_{l, k} \ldots \ldots \ldots \ldots \ldots$. . . . 77

4.2 .3 Resultados . . . . . . . . . . . . . . . . . . . 83

$4.3 \mathrm{O}$ modelo GL em $d$ dimensões $\ldots \ldots \ldots$. . . . . . . . . . . . 84

4.3.1 Aplicação do método de expansão em série . . . . . . . . . 85

4.3.2 Cálculo dos coeficientes $A_{l, \mathbf{r}} \ldots \ldots \ldots \ldots$. . . . 87

4.3 .3 Resultados . . . . . . . . . . . . . . . . . 96

4.4 Expansão em série para o modelo de Glauber 2D . . . . . . . . . 100

4.4 .1 Expansão em série perturbativa . . . . . . . . . . . . . . . 104

4.4.2 Construção numérica da série. Resultados . . . . . . . . . 106

5 Considerações finais $\quad 111$

$\begin{array}{ll}\text { A Algumas expansões em série } & 115\end{array}$

$\begin{array}{ll}\text { B O algoritmo de Gosper-Zeilberger } & 117\end{array}$ 


\section{Capítulo 1}

\section{Introdução}

Sistemas reais podem apresentar uma evolução temporal irreversível quando em contato com os arredores, seja porque não atingiram o equilíbrio, seja porque tenham alcançado um estado estacionário fora do equilíbrio termodinâmico. Uma possível abordagem para a descrição de tais sistemas supõe que o estado $\sigma$ do sistema segue uma trajetória estocástica markoviana conforme o sistema evolui. A descrição de um processo estocástico envolve a probabilidade $P(\sigma, t)$ de se encontrar o sistema na configuração $\sigma$ como uma função do tempo. Para sistemas em equilíbrio térmico à temperatura $T$,

$$
P(\sigma)=\frac{1}{Z} \exp \left\{-\frac{\mathcal{H}(\sigma)}{k_{B} T}\right\}
$$

onde $\mathcal{H}(\sigma)$ é o hamiltoniano calculado para a configuração $\sigma[1,2]$.

Para a maioria dos modelos estocásticos, não existe uma descrição por meio de um hamiltoniano. Entretanto, podemos descrever a evolução temporal para a probabilidade de encontrarmos o sistema na configuração $\sigma$ utilizando a equação mestra $[3,4,5]$ :

$$
\frac{d}{d t} P(\sigma, t)=\sum_{\sigma^{\prime}(\neq \sigma)}\left\{W\left(\sigma, \sigma^{\prime}\right) P\left(\sigma^{\prime}, t\right)-W\left(\sigma^{\prime}, \sigma\right) P(\sigma, t)\right\}
$$

em que $W\left(\sigma^{\prime}, \sigma\right)$ é a taxa de transição da configuração $\sigma$ para a configuração $\sigma^{\prime}$. Um modelo estocástico pode ser definido impondo-se um conjunto de configurações possíveis e o conjunto de taxas de transição conectando estas configurações. Para um sistema em equilíbrio termodinâmico descrito por uma equação mestra, a probabilidade $P(\sigma)$ não varia no tempo e obedece à relação de balanceamento detalhado, 
que é obtida tomando a equação mestra (1.2) e igualando a zero o termo dentro da somatória, para quaisquer configurações $\sigma$ e $\sigma^{\prime}$ :

$$
W\left(\sigma^{\prime}, \sigma\right) P(\sigma)=W\left(\sigma, \sigma^{\prime}\right) P\left(\sigma^{\prime}\right)
$$

Podemos interpretar esta condição dizendo que, quando existe o equilíbrio termodinâmico, o número de transições entre duas configurações $\sigma$ e $\sigma^{\prime}$ durante o intervalo de tempo $\Delta t$, que é igual a $W\left(\sigma^{\prime}, \sigma\right) P(\sigma) \Delta t$, não deve ser alterado pela troca $\sigma \leftrightarrow \sigma^{\prime}$, independentemente de $\Delta t$.

Modelos para os quais não existe uma função $P(\sigma)$ satisfazendo a relação de balanceamento detalhado não apresentam equilíbrio termodinâmico. Uma classe de modelos para a qual não existe o estado de equilíbrio termodinâmico, mas apenas um estado estacionário, consiste nos modelos que apresentam estados absorventes: estados $\sigma$ tais que $W\left(\sigma^{\prime}, \sigma\right)=0$ para qualquer outro estado $\sigma^{\prime}$. É importante distinguir, dentre os modelos que apresentam estados absorventes, aqueles que apresentam um número finito de estados absorventes, como o processo de contato [5, 6, 7], visto brevemente no capítulo 2, e aqueles que apresentam um número infinitamente grande de estados absorventes $[8,10,9,11,12,13]$ (o número de estados absorventes aumenta com o tamanho do sistema), como a classe de modelos de pilha de areia, vista no capítulo 3.

Propriedades dinâmicas e estacionárias de modelos estocásticos podem ser obtidas a partir da equação mestra. A fim de considerar alguns métodos analíticos, primeiramente escrevemos a matriz de evolução $W$, cujos elementos são as taxas de transição $W\left(\sigma^{\prime}, \sigma\right)$, como será visto no capítulo 4 . Descobrindo os autovalores e autovetores de $W$, podemos utilizá-los para obter propriedades dinâmicas, tais como a probabilidade de sobrevivência de uma configuração inicial $\sigma$ antes de cair no estado absorvente e a função de correlação temporal. É possível mostrar [5] que o autovetor à direita de $W$ correspondente ao autovalor nulo tem como componentes a probabilidade estacionária de cada configuração $P_{e}(\sigma)$. Assim, utilizando essa descrição, podemos encontrar a probabilidade estacionária de um modelo estocástico ou funções que descrevem o estado estacionário, como a susceptibilidade de um modelo magnético.

Poucos modelos podem ser resolvidos de forma exata, de forma que devemos dispor de esquemas aproximativos para obter as propriedades estacionárias do modelo. Um esquema bastante conhecido é a aproximação de campo médio $[1,5]$, que 
despreza as interações entre sítios individuais e tenta descrever o sistema através de interações entre todos os sítios. Utilizando as aproximações de campo médio até a $n$-ésima ordem, obtemos um sistema de equações acoplando as densidades de aglomerados de até $n$ sítios, que com sorte pode ser resolvido numericamente. Outra opção é, após implantar a aproximação correspondente, integrar numericamente as equações de evolução temporal para obter os valores médios estacionários.

Métodos numéricos para o estudo de modelos estocásticos tem tido bastante expressão nas últimas décadas, graças ao avanço notável do poder computacional disponível para a pesquisa. Métodos numéricos são utilizados para simular a evolução temporal de um processo a partir de um conjunto de condições iniciais diferentes, para que seja possível observar a entrada do sistema no estado estacionário e calcular valores médios estacionários $[14,15]$. Na criticalidade [5, 16], o tempo necessário para simular um modelo aumenta consideravelmente, já que nessa região observamse correlações entre todos os sítios da rede e deve-se observar o sistema por mais tempo antes que seja possível concluir que ele está no estado estacionário, além de ser necessário simular o sistema no estado estacionário por mais tempo, para que a amostra da trajetória do sistema no espaço de fase seja mais representativa. Entre as técnicas desenvolvidas para analisar o estado crítico, destaca-se a análise de escala finita (finite-size scaling $[15,17]$ ), que recolhe resultados obtidos em sistemas com tamanhos diferentes perto da criticalidade para obter informações sobre a transição e classificar o sistema em alguma classe de universalidade.

Nesta tese, vamos expor alguns modelos estocásticos ligados a áreas de pesquisa ativas atualmente. No capítulo 2, estudamos o processo de contato [6], um dos modelos estocásticos mais simples a exibir uma transição de fase em uma dimensão. A localização do ponto crítico $\alpha_{c}$ e o valor do expoente crítico $\beta$, relacionado à queda da densidade de partículas, são conhecidos com bastante exatidão por meio de simulações numéricas [5] e de análise de expansões em série perturbativas [7]. Nós exibimos um novo método de cálculo dos termos da expansão em série, capaz de calcular $\alpha_{c}$ e $\beta$ com precisão equivalente à dos demais métodos, e mostramos que os resultados obtidos por nosso método são consistentes.

A seguir, no capítulo 3, estudamos modelos de pilha de areia, bastante investigados atualmente devido a suas conexões com o fenômeno de criticalidade autoorganizada $[8,10,9]$. Nós definimos um novo modelo de pilha de areia, introduzindo uma taxa de transição com assimetria espacial, regida por um parâmetro $p$. Quando $p=0,5$, este modelo é idêntico a um modelo estudado por Dickman, Tomé e Oli- 
veira [12] e Dickman [18]. Estudamos algumas de suas propriedades, tais como a variação do ponto crítico e dos expoentes críticos $\beta$ e $\nu_{\perp}$ (relacionado à divergência do comprimento de correlação nas proximidades do ponto crítico [16]) com $p$ e a variação do fluxo estacionário de partículas com $p$ e a densidade $\rho$ por aproximações de campo médio e simulação. Comparando os resultados que ibtivemos para o novo modelo quando $p \rightarrow 0,5$ com os resultados de Dickman, notamos que ocorre uma mudança da classe de universalidade do sistema, uma vez que $\beta$ e $\nu_{\perp}$ assumem valores diferentes para $p=0,5$ e $p \neq 0,5$. Esse trabalho deu origem a um artigo, publicado na revista Journal of Physics A: Mathematical and Theoretical em 2009.

No capítulo 4, estudamos o modelo de Glauber linear em $d$ dimensões analiticamente, calculando a susceptibilidade magnética a campo nulo $\chi$ por meio de uma expansão em série perturbativa[5]. Este modelo foi anteriormente estudado por Oliveira [19], e $\chi$ foi obtida por meio da função de Green na rede. Neste trabalho, mostramos como o método de expansão em série converte o problema do cálculo de $\chi$ em uma relação de recorrência, a qual pode ser resolvida apenas para $d=1$; entretanto, várias identidades envolvendo $\chi$ podem ser estabelecidas quando $d>1$. Um artigo referente a esse trabalho está sendo escrito no momento. Após esse cálculo, utilizando a mesma técnica, introduzimos um método numérico para se construir uma expansão em série temporal para o modelo de Glauber [20] em duas dimensões (que reproduz a probabilidade estacionária de um sistema de Ising [21, 22]). Os resultados obtidos nessa seção ainda são provisórios, devido à dificuldade existente em calcular os coeficientes da série. Finalmente, no capítulo 5, faremos algumas considerações finais sobre os modelos estudados. 


\section{Capítulo 2}

\section{Estudo do processo de contato por expansão em série}

Neste capítulo, vamos fazer uma introdução aos métodos de estudo de sistemas estocásticos, estudando um modelo estocástico não trivial cujas propriedades críticas são conhecidas - o processo de contato em uma dimensão.

\subsection{O processo de contato}

O processo de contato é um dos modelos mais simples dentro da Física Estatística fora do equilíbrio, sendo possivelmente o modelo mais simples que exibe transição de fase em uma dimensão. Ele foi introduzido primeiramente por Harris [6], como um toy model para o estudo de propagação de epidemias.

O processo de contato é definido numa rede hiper-cúbica em $d$ dimensões. Sítios na rede apresentam dois estados possíveis: infectado (contribui para a propagação da epidemia) e sadio (pode ser infectado se entrar em contato com um sítio infectado). Na dinâmica deste processo, atualiza-se o estado de um sítio por vez: um sítio sadio pode tornar-se infectado se ao menos um de seus vizinhos mais próximos estiver infectado, e a probabilidade de ser infectado é proprocional ao número de vizinhos infectados. Um sítio infectado, por sua vez, pode se curar espontaneamente, independentemente da influência dos vizinhos. A doença não causa imunidade, de modo que um sítio inicialmente sadio tem propriedades idênticas às de um sítio que foi infectado e curou-se. De acordo com estas regras, se em algum instante de tempo não houver nenhum sítio infectado, a epidemia extingue-se, porque não 
Tabela 2.1: Taxas de transição para o processo de contato unidimensional.

\begin{tabular}{|c|c||c|c|}
\hline Criação & Taxa & Aniquilação & Taxa \\
\hline $100 \rightarrow 110$ & $1 / 2$ & & \\
$001 \rightarrow 011$ & $1 / 2$ & $1 \rightarrow 0$ & $\alpha$ \\
$101 \rightarrow 111$ & 1 & & \\
\hline
\end{tabular}

existem meios de reiniciar a epidemia na ausência de sítios infectados.

O processo de contato é normalmente identificado com um modelo de criação e aniquilação de partículas: um sítio sadio é associado a um sítio vazio, enquanto um sítio infectado é associado a um sítio ocupado por uma partícula. A propagação da doença é equivalente à criação de uma partícula em um sítio vazio vizinho de ao menos um sítio ocupado, enquanto a cura é equivalente à aniquilação de uma partícula.

Seja $\eta_{i}=0,1$ a variável de ocupação de um sítio da rede, com $1 \leq i \leq L^{d}$. Uma configuração $\eta$ é determinada pela sequência de variáveis $\eta_{i}$ em cada sítio. $\mathrm{O}$ processo de criação ocorre com taxa $n / 2 d$, onde $n$ é o número de sítios vizinhos ocupados, enquanto o processo de aniquilação ocorre com taxa $\alpha, 0 \leq \alpha<\infty^{1}$. Tomando $d=1$, as taxas de criação e aniquilação para as possíveis sequências iniciais de três e um sítio, respectivamente, aparecem na tabela 2.1.

Para valores grandes de $\alpha$, o processo de aniquilação ocorre mais frequentemente que o processo de criação, de modo que no final de um certo tempo todas as partículas deixarão a rede, e o sistema cairá no estado absorvente (fim da epidemia). Para valores pequenos de $\alpha$, o processo de criação ocorre mais frequentemente que o processo de aniquilação, de modo que o sistema permanece indefinidamente no estado ativo (a epidemia nunca se extingue). Assim, para um certo valor crítico $\alpha_{c}$, deve ocorrer uma transição entre a fase estacionária ativa e a fase absorvente.

$\mathrm{O}$ valor de $\alpha_{c}$ varia conforme a dimensão do modelo. Uma primeira estimativa para $\alpha_{c}$ pode ser obtida por aproximação de campo médio simples. Para $d=1$, usando a tabela 2.1, a equação de evolução temporal para a densidade de partículas $\rho=P_{1}$ é:

$$
\frac{d}{d t} P_{1}=-\alpha P_{1}+P_{101}+\frac{P_{100}+P_{001}}{2}
$$

\footnotetext{
${ }^{1}$ Esta não é a única escolha possível para as taxas de criação e aniquilação. Muitos outros trabalhos tomam a taxa de aniquilação igual a 1, enquanto a taxa de criação é igual a $\frac{n}{2 d} \lambda$, $0 \leq \lambda<\infty$. Com essa escolha, a fase ativa ocorre para $\lambda>\lambda_{c}$
} 
Acima, $P_{101}$ representa a probabilidade de que uma sequência de três sítios dentro da rede seja ocupada pela configuração 101. As outras grandezas possuem significado análogo.

A equação acima pode ser reescrita utilizando variáveis dependentes de menos sítios. Utilizamos as seguintes condições de normalização:

$$
\begin{aligned}
P_{1} & =1-P_{0} \\
P_{01} & =P_{10}=P_{0}-P_{00} \\
P_{001} & =P_{100}=P_{00}-P_{000} \\
P_{101} & =P_{01}-P_{001}=P_{0}-2 P_{00}+P_{000}
\end{aligned}
$$

para reescrever (2.1) na forma:

$$
\frac{d}{d t} P_{1}=-\alpha+(1+\alpha) P_{0}-P_{00}
$$

Vemos que a equação de evolução temporal de $P_{1}$ depende da probabilidade de uma sequência de 2 sítios. Se escrevermos a equação de evolução temporal para $P_{00}$, veremos que probabilidades de três sítios são envolvidas. De forma geral, a equação de evolução temporal para uma probabilidade de $n$ sítios depende de probabilidades de $n+1$ sítios, sendo necessário um truncamento da sequência de equações.

A aproximação de campo médio de $n$-ésima ordem [5], $n \leq 1$, é um método de truncamento da sequência de equações bastante utilizado. Na aproximação de campo médio simples $(n=1)$, escrevemos todas as grandezas dependentes de 2 ou mais sítios como o produto das probabilidades de um único sítio $P_{1}$ e $P_{0}$, eliminando todas as correlações entre sítios que possam existir. Aplicando essa aproximação à equação (2.6) e tomando $\frac{d}{d t} P_{1}=0$ (descrição do estado estacionário), obtemos:

$$
\alpha-(1+\alpha) P_{0}+P_{0}^{2}=0
$$

que admite duas soluções:

$$
P_{0}= \begin{cases}\alpha & (\text { estado ativo }), \text { ou } \\ 1 & (\text { estado absorvente, } \operatorname{com} \rho=0)\end{cases}
$$

Assim, a aproximação de campo médio simples descreve um sistema com duas 
fases: uma fase ativa, que ocorre no intervalo $0 \leq \alpha \leq 1$, e uma fase absorvente, para $\alpha>1$. A transição de fase pode ser caracterizada por um expoente crítico $\beta$ [16], que descreve o comportamento da densidade de partículas perto do ponto crítico $\alpha_{c}=1$ :

$$
\rho \sim\left|\alpha-\alpha_{c}\right|^{\beta}
$$

Perto do ponto crítico, na aproximação de campo médio simples, podemos escrever:

$$
\rho=1-P_{0}=(1-\alpha)=\left(\alpha_{c}-\alpha\right)
$$

de modo que esta aproximação fornece $\beta=1$.

\subsection{Hierarquia de equações e expansão em série}

É possível obter $\alpha_{c}$ e $\beta$ por meio de simulaçõs numéricas. Os valores aceitos atualmente para o processo de contato em uma dimensão são $\alpha_{c}=0,30322$ e $\beta=0,2765$ [5]. Uma vez que a aproximação que utilizamos na seção anterior despreza correlações entre sítios próximos, os resultados obtidos para $\alpha_{c}$ e $\beta$ são diferentes dos obtidos por simulação.

Para incluirmos tais correlações, vamos escrever uma expansão supercrítica (ou seja, válida na fase ativa) da variável $P_{1}$ em série de potências do parâmetro $\alpha$. Essa expansão deve ser acurada para $\alpha$ pequeno (ou seja, quando a densidade de partículas no estado estacionário é grande), perdendo a validade quando $\alpha$ cresce. À medida que mais termos da expansão são escritos, o raio de convergência da expansão deve crescer, de modo que deve ser possível extrair informações a respeito do ponto crítico.

Primeiramente, vamos expor algumas propriedades das probabilidades de agrupamentos de $n$ sítios:

- Quando $\alpha$ é pequeno, a densidade de sítios vazios no estado estacionário deve ser pequena, porque processos de aniquilação são raros. Assim, o número de pares de sítios duplamente vazios deve ser muito menor que o número de sítios vazios, uma vez que, se um sítio vazio é escolhido ao acaso, é bem mais provável que seu primeiro vizinho esteja ocupado.

- Se $P_{0}$ é, em primeira ordem, proporcional a $\alpha$ (como sugerido pela aproximação de campo médio simples), então $P_{00}$ deve ser proporcional a $\alpha^{2}$ em primeira 
ordem:

$$
P_{00}=c_{2}^{(00)} \alpha^{2}+O\left(\alpha^{3}\right)
$$

$\mathrm{Na}$ aproximação de campo médio simples, caracterizada pela ausência de correlações entre sítios vizinhos, impomos $c_{2}^{(00)}=1$. Incluindo de forma correta a correlação entre dois sítios no estado estacionário, devemos obter um coeficiente diferente.

- Um raciocínio análogo mostra que, dada uma sequência $x$ de $M$ sítios, dos quais $N$ são vazios e $M-N$ são ocupados, a probabilidade $P_{x}$ dessa sequência no estado estacionário pode ser escrita como:

$$
P_{x}=c_{N}^{(x)} \alpha^{N}+O\left(\alpha^{N+1}\right)
$$

Essa expressão para $P_{x}$ deve ser mais acurada para $\alpha$ pequeno.

Nosso objetivo nesta seção é escrever uma expansão em série para $P_{1}$, na forma:

$$
P_{1}=\sum_{n=0} c_{n}^{(1)} \alpha^{n}
$$

Para determinar os coeficientes $c_{n}^{(1)}$, vamos impor as condições a seguir:

- Cada grandeza $P_{x}$, onde $x$ é uma sequência contínua de sítios vazios e ocupados, pode ser escrita como uma expansão em série de potências de $\alpha$. O primeiro termo não-nulo da série é o de ordem igual ao número de sítios vazios da sequência. Exemplos:

$$
\begin{aligned}
P_{0} & =\sum_{n=1}^{\infty} c_{n}^{(0)} \alpha^{n} & P_{00} & =\sum_{n=2}^{\infty} c_{n}^{(00)} \alpha^{n} \\
P_{000} & =\sum_{n=3}^{\infty} c_{n}^{(000)} \alpha^{n} & P_{010} & =\sum_{n=2}^{\infty} c_{n}^{(010)} \alpha^{n} \ldots
\end{aligned}
$$

- Utilizamos condições de normalização, como (2.2), (2.3), (2.4) e (2.5), para diminuir o número de coeficientes independentes. Devido a essas condições de normalização, nossos cálculos vão envolver apenas uma sequência de 1 sítio, uma sequência de 2 sítios, duas sequências de 3 sítios, ... As sequências escolhidas para o cálculo são aquelas que começam e terminam com um sítio vazio 
$(0,00,000,010, \ldots)$, porque o número de coeficientes envolvidos no total é menor $^{2}$.

- Escrevemos as equações de evolução para as probabilidades da sequências escolhidas como variáveis independentes. Como vamos mostrar a seguir, para calcular o coeficientes de $P_{1}$ de ordem $n$, precisamos das equações de evolução temporal de sequências com no máximo $n$ sítios.

- Substituímos as expansões (2.14) nas equações de evolução temporal e igualamos a derivada temporal a zero. Obtemos equações envolvendo coeficientes de ordens diferentes, uma vez que $\alpha$ aparece explicitamente nas equações de evolução, multiplicando os termos vindos de processos de aniquilação. Analisando a estrutura das equações de evolução, pode-se ver que é possível interromper o cálculo em qualquer ordem $n$, obtendo os coeficientes $c_{l}^{(1)}, l \leq n$. Os coeficientes obtidos até a ordem $n$ são idênticos aos obtidos por aproximação de campo médio de ordem $n$; entretanto, se expandirmos $P_{1}$ obtido pela aproximação de ordem $n$ até potências de ordem maior que $n$, os coeficientes passam a ser distintos. Resolvendo essas equações, obtemos $c_{n}^{(0)}$ e, utilizando a relação de normalização para $P_{1}$, obtemos $c_{n}^{(1)}$.

Como exemplo, vejamos como calcular os coeficientes $c_{n}^{(1)}$ para $n \leq 3$. Para $\alpha=$ $0, P_{1}=1$ (não existem sítios vazios no estado estacionário se a taxa de aniquilação é nula). Logo, $c_{0}^{(1)}=1$.

Para $n=1$, tomamos a equação de evolução temporal de $P_{1}$ após aplicar as relações de normalização (2.6), e substituímos as expansões em série (2.14). Obtemos a relação entre os coeficientes:

$$
c_{n}^{(0)}=-c_{n-1}^{(0)}+c_{n}^{(00)}+\delta_{n, 1}, \quad n \geq 1
$$

Tomando $n=1$, impomos $c_{0}^{(0)}=c_{1}^{(00)}=0$, obtendo

$$
c_{1}^{(0)}=1, \quad c_{1}^{(1)}=-c_{1}^{(0)}=-1
$$

\footnotetext{
${ }^{2}$ Entretanto, é possível utilizar outros critérios de escolha. Por exemplo, é possível usar a mesma técnica de expansão em série usando $\lambda$ - relacionado a uma taxa de criação variável como parâmetro de expansão. Nesse caso, a escolha mais econômica de variáveis inclui apenas as sequências que começam e terminam com 1
} 
Para $n=2$, precisamos da equação de evolução temporal de $P_{00}$, dada por:

$$
\frac{d}{d t} P_{00}=2 \alpha P_{0}-(2 \alpha+1) P_{00}+P_{000}
$$

uma vez que as demais probabilidades de dois sítios podem ser obtidas impondo condições de normalização.

Substituindo as expansões (2.14) em (2.17), obtemos uma nova relação envolvendo os coeficientes:

$$
c_{n}^{(00)}=2 c_{n-1}^{(0)}-2 c_{n-1}^{(00)}+c_{n}^{(000)}, \quad n \geq 2
$$

Fixando $n=2$, obtemos a partir de (2.18) e (2.15) os resultados:

$$
\begin{aligned}
c_{2}^{(00)} & =2 c_{1}^{(0)}=2 \\
c_{2}^{(0)} & =-c_{1}^{(0)}+c_{2}^{(00)}=1
\end{aligned}
$$

de modo que $c_{2}^{(1)}=-1$.

Para $n=3$, precisamos de mais duas equações. Além da equação para a variável $P_{000}$, a qual aparece na equação de evolução temporal de $P_{00}$, vamos escrever a equação de evolução temporal de $P_{010}$ (que aparece na equação anterior após aplicarmos todas as condições de normalização relevantes):

$$
\begin{aligned}
& \frac{d}{d t} P_{000}=2 \alpha P_{00}-(2 \alpha+1) P_{000}+\alpha P_{010}+P_{0000} \\
& \frac{d}{d t} P_{010}=2 \alpha P_{0}-2 \alpha P_{00}-(3 \alpha+2) P_{010}+P_{0010}
\end{aligned}
$$

Fazemos as substituições (2.14) nas duas equações acima, obtendo duas relações entre coeficientes:

$$
\begin{array}{ll}
c_{n}^{(000)}=2 c_{n-1}^{(00)}-2 c_{n-1}^{(000)}+c_{n-1}^{(010)}+c_{n}^{(0000)}, & n \geq 3 \\
c_{n}^{(010)}=c_{n-1}^{(0)}-c_{n-1}^{(00)}-\frac{3}{2} c_{n-1}^{(010)}+\frac{1}{2} c_{n}^{(0010)}, & n \geq 2
\end{array}
$$

Notamos que, para obter $c_{3}^{(0)}$, não é necessário calcular $c_{3}^{(010)}$, mas $c_{2}^{(010)}$. Também vemos que existe uma ordem precisa para o cálculo dos coeficientes: $c_{2}^{(010)} \rightarrow c_{3}^{(000)} \rightarrow$ 
$c_{3}^{(00)} \rightarrow c_{3}^{(0)}$. Resolvendo $(2.24),(2.23),(2.18)$ e (2.15), obtemos:

$$
\begin{aligned}
c_{2}^{(010)} & =c_{1}^{(0)}=1 \\
c_{3}^{(000)} & =2 c_{2}^{(00)}+c_{2}^{(010)}=5 \\
c_{3}^{(00)} & =2 c_{2}^{(0)}-2 c_{2}^{(00)}+c_{3}^{(000)}=3 \\
c_{3}^{(0)} & =-c_{2}^{(0)}+c_{3}^{(00)}=2
\end{aligned}
$$

de modo que $c_{3}^{(1)}=-2$. Assim, a expansão em série de $P_{1}$ em potências de $\alpha$ até a $3^{\mathrm{a}}$ ordem é:

$$
P_{1}=1-\alpha-\alpha^{2}-2 \alpha^{3}+O\left(\alpha^{4}\right)
$$

Quando escrevemos as relações de recorrência (2.15), (2.18), (2.23) e (2.24), colocamos o coeficiente $c_{n}^{(x)}$ (onde $x$ é a sequência de sítios para a qual construímos a equação de evolução temporal) em função de outros coeficientes. De fato, ao escrevermos a equação de evolução temporal para a sequência $x$, o termo $P_{x}$ sempre aparece, devido a processos de criação envolvendo o primeiro e o último sítio da sequência, os quais são vazios, devido ao critério que utilizamos para escolher as variáveis independentes (obviamente, processos de criação no interior da sequência e de aniquilação também podem contribuir para a presença de $P_{x}$ na equação de evolução temporal)

É possível mostrar que os demais coeficientes podem ser classificados em uma das classes a seguir:

- Coeficientes de sequências $x^{\prime}$ com o mesmo número de sítios e um sítio ocupado a mais ou a menos que a sequência $x$, de ordem $n-1$. Esses termos aparecem devido a processos de aniquilação de partículas no interior da sequência. Exemplo: devido à transição $0110 \rightarrow 0010$, que ocorre com taxa $\alpha$, existe um termo $\alpha P_{0110}$ na equação de evolução temporal de $P_{0010}$, e um termo $-\alpha P_{0110}$ na equação de $P_{0010}$.

- Coeficientes de sequências $x^{\prime}$ com um sítio a mais que a sequência $x$, de ordem $n$. O sítio extra é um sítio vazio, devido à escolha de variáveis. Esses termos aparecem devido a processos de criação de partículas no primeiro e último sítio da sequência. Exemplo: a transição $000 \rightarrow 001$ ocorre com probabilidade $P_{0001} / 2=\left(P_{000}-P_{0000}\right) / 2$, de modo que aparece o termo $P_{0000}$ na equação de evolução temporal de $P_{000}$. 
- Coeficientes de sequências $x^{\prime}$ com um sítio ocupado a mais ou a menos, de ordem $n$. Esses termos ocorrem devido a processo de criação no interior da sequência (ou seja, em qualquer um dos sítios, exceto o primeiro e o último), desde que exista ao menos um sítio ocupado. Ex: devido à transição $0010 \rightarrow$ 0110, que ocorre com taxa $1 / 2$, existe um termo $P_{0010} / 2$ na equação de evolução temporal de $P_{0110}$ e um termo $-P_{0010} / 2$ na equação de $P_{0010}$. Em particular, se a sequência $x$ possui sítios vazios apenas nas extremidades, as sequências $x^{\prime}$ possíveis são apenas aquelas com um sítios ocupado a menos.

- Coeficientes de sequências $x^{\prime}$ com sítios a menos, de ordem $n-1$. Tais termos são obtidos devido a processo de aniquilação envolvendo o primeiro ou o último sítio da sequência, e envolvem o uso de condições de normalização. Ex: devido à transição $01011 \rightarrow 01010$, existe um termo $\alpha P_{01011}$ na equação de evolução temporal de $P_{01010}$, o qual pode ser reescrito como $\alpha\left(P_{010}-P_{0100}-P_{01010}\right)$.

Assim, para sermos capazes de calcular $c_{n}^{(0)}$, precisamos calcular termos intermediários $c_{n^{\prime}}^{(x)}$, onde $x$ pode ser uma sequência com mais sítios, ou com um número diferente de sítios ocupados, ou a mesma sequência, de ordem inferior. Escrevendo as equações de recorrência de modo a destacar o coeficiente de ordem $n$ da sequência $x$ de cada equação de evolução temporal, o cálculo de cada orde envolve uma série de cálculos intermediários que acaba quando todos os coeficientes necessários já foram calculados em alguma ordem anterior a $n$ ou são nulos, porque correspondem a sequências cujo número de sítios vazios é maior do que $n$.

\subsection{Análise de Padé}

Nesta seção, veremos como analisar uma expansão em série com número finito de termos conhecidos de uma grandeza que apresenta comportamento crítico, de modo a obter uma estimativa para o ponto crítico e o expoente crítico correspondente, usando o método de aproximantes de Padé [23]. Como exemplo, vamos calcular uma aproximação para $\alpha_{c}$ e $\beta$ do processo de contato unidimensional a partir de (2.29).

Perto do ponto crítico $\alpha_{c}, \rho$ deve se anular de acordo com (2.9). Se este comportamento é válido, então podemos obter estimativas de $\rho_{c}$ e do expoente $\beta$ analisando 
a série em potências de $\alpha$ de:

$$
\frac{d}{d \alpha} \ln \rho=\frac{d}{d \alpha} \ln \left|\alpha-\alpha_{c}\right|^{\beta}=\frac{\beta}{\alpha-\alpha_{c}}
$$

Da equação acima, vemos que $\alpha_{c}$ corresponde a um polo da expansão em série do operador $\frac{d}{d \alpha} \ln$ aplicado à série de potências de $\alpha$ de $P_{1}$, e o resíduo correspondente é igual a $\beta$. Para obter o polo e o resíduo a partir de uma série de potências em $\alpha$, um dos métodos utilizados atualmente na análise de modelos estocásticos é a análise de Padé, também conhecida como método de aproximantes de Padé.

Introduzindo brevemente o método de aproximantes de Padé, ele consiste em escrever uma função racional do parâmetro $\alpha$ cuja expansão em série de Taylor na variável $\alpha$ seja idêntica à série que estamos estudando até a potência desejada. Se $f(\alpha)=\sum_{n=0}^{N} a_{n} \alpha^{n}$ é a série que desejamos aproximar, o aproximante de Padé $F(\alpha)$ de ordem $[L, M]$ é dado por:

$$
F(\alpha)=\frac{P(\alpha)}{Q(\alpha)}=\frac{\sum_{l=0}^{L} p_{l} \alpha^{l}}{1+\sum_{m=1}^{M} q_{m} \alpha^{m}}
$$

onde os coeficientes $p_{l}$ e $q_{m}$ são tais que a condição a seguir seja satisfeita até a ordem $L+M$ :

$$
Q(\alpha) f(\alpha)=P(\alpha) \Rightarrow\left(1+\sum_{m=1}^{M} q_{m} \alpha^{m}\right)\left(\sum_{n=0}^{N} a_{n} \alpha^{n}\right)=\sum_{l=0}^{L} p_{l} \alpha^{l}
$$

ou seja, os coeficientes de $\alpha^{l}$ de cada lado da igualdade (2.32) devem ser idênticos no intervalo $0 \leq l \leq L+M$, satisfazendo a $L+M+1$ equações simultaneamente. Tradicionalmente, na análise de modelos estocásticos, tomam-se os aproximantes de Padé de ordem $[L, L],[L, L+1]$ e/ou $[L, L-1]$. Tais aproximantes

Com o método de aproximantes de Padé, podemos estender a convergência de uma expansão em série de uma função analítica para fora do raio de convergência original. Isso ocorre, simplificadamente, porque os aproximantes de Padé têm a capacidade de modelar de forma eficaz polos simples e múltiplos de uma função, fornecendo uma continuação analítica para uma série cuja região de convergência é limitada por polos.

É um fato conhecido que as propriedades de convergência dos aproximantes de Padé são conhecidas apenas para algumas classes de funções: ainda não é possível 
determinar com certeza qual a região onde o aproximante de Padé de ordem $[L, M]$ converge para o resultado correto, ou se ele converge de fato para uma função real qualquer (ver discussão em [23]). Entretanto, a análise de modelos estocásticos vem mostrando nos últimos anos que, na grande maioria dos problemas estudados até agora, a análise de Padé fornece bons resultados.

Agora, vamos ilustrar obter estimativas para $\alpha_{c}$ e $\beta$ a partir da série de quatro termos (2.29) para $\rho$. Em primeiro lugar, expandimos o logaritmo natural de (2.29) em série de potências, utilizando a expansão de Taylor:

$$
\ln (1+z)=\sum_{n=1}^{\infty}(-1)^{n-1} \frac{z^{n}}{n}
$$

Obtemos:

$$
\begin{aligned}
\ln \rho=\ln \left(1-\alpha-\alpha^{2}-2 \alpha^{3}\right) & =\left(-\alpha-\alpha^{2}-2 \alpha^{3}\right)-\frac{1}{2}\left(-\alpha-\alpha^{2}\right)^{2}+ \\
+ & \frac{1}{3}(-\alpha)^{3}+O\left(\alpha^{4}\right)=-\alpha-\frac{3}{2} \alpha^{2}-\frac{10}{3} \alpha^{3}+O\left(\alpha^{4}\right)
\end{aligned}
$$

Derivando esta série, obtemos:

$$
\frac{d}{d \alpha} \ln \rho=-1-3 \alpha-10 \alpha^{2}+O\left(\alpha^{3}\right)
$$

Agora, vamos calcular aproximantes de Padé para a série (2.35). Estimamos que a ordem dos aproximantes mais adequada para este cálculo é [1,1], ou seja, os polinômios que vamos utilizar para o cálculo têm grau 1 :

$$
\begin{aligned}
& P(\alpha)=p_{0}+p_{1} \alpha \\
& Q(\alpha)=1+q_{1} \alpha
\end{aligned}
$$

Obtemos os coeficientes $p_{0}, p_{1}$ e $q_{1}$ impondo que (2.32) seja verdadeira até o coeficiente de $\alpha^{2}$ :

$$
\left(1+q_{1} \alpha\right)\left(-1-3 \alpha-10 \alpha^{2}\right)=p_{0}+p_{1} \alpha
$$

Os coeficientes obtidos por este método são $p_{0}=-1, p_{1}=1 / 3$ e $q_{1}=-10 / 3$, de 
modo que o aproximante de Padé de ordem $[1,1]$ é:

$$
F(\alpha)=\frac{-1+\frac{1}{3} \alpha}{1-\frac{10}{3} \alpha}
$$

A função $F(\alpha)$ possui um polo simples, o qual identificamos com o ponto crítico: $\alpha_{c}=3 / 10=0,3$. Obtemos o expoente crítico $\beta$ como o resíduo de $F$ calculado em $\alpha_{c}$ :

$$
\beta=\lim _{\alpha \rightarrow \alpha_{c}}\left(\alpha-\alpha_{c}\right) F(\alpha)=\frac{3}{10}\left(1-\frac{\alpha_{c}}{3}\right)=0,27
$$

Jensen e Dickman [7] também calcularam a taxa de aniquilação crítica e o expoente crítico $\beta$ para o processo de contato através da análise de uma expansão em série, obtendo os valores $\alpha_{c}=0,303228(2)$ e $\beta=0,27690(5)$. Comparando estes valores com os nossos, vemos que o método de aproximantes de Padé é capaz de produzir boas estimativas com pouco poder computacional. A seguir, vamos mostrar que é possível melhorar nossas estimativas para $\alpha_{c}$ e $\beta$ calculando mais termos da série de $\rho$.

\subsection{Implementação do programa. Resultados}

Dos resultados da seção anterior, vemos que é necessário obter um número maior de termos da série de $\rho$ para obter boas estimativas de $\alpha_{c}$ e $\beta$ pela análise de Padé. Entretanto, à medida que cresce o número de termos, os cálculos envolvidos na obtenção dos coeficientes $c_{n}^{(0)}$ e dos coeficientes dos aproximantes de Padé $p_{l}$ e $q_{m}$ tornam-se mais complexos: o primeiro, porque aumenta o número de coeficientes intermediários que são diferentes de zero e devem ser calculados, e o segundo, porque é necessário resolver um sistema de equações lineares de ordem cada vez maior.

Para automatizar o processo e evitar a ocorrência de erros, torna-se necessário escrever programas que cumpram os seguintes objetivos:

- Identificar as variáveis $P_{x}$ necessárias ao cálculo de $c_{n}^{(0)}$. Escrever as equações de evolução temporal para cada variável necessária e identificar qual o primeiro coeficiente não-nulo de sua expansão em série no parâmetro $\alpha$;

- Resolver as relações para os coeficientes obtidas ao substituir as expansões em série de todas as variáveis nas equações de evolução temporal no estado estacionário, até obter $c_{n}^{(0)}$; 
- Obter a expansão em série para $\frac{d}{d \alpha} \ln \rho$;

- Achar os coeficientes $p_{l}, q_{m}$ do aproximante de Padé de ordem $[n / 2, n / 2]$ (se $n$ for par), $[(n+1) / 2,(n-1) / 2]$ ou $[(n-1) / 2,(n+1) / 2]$ (se $n$ for ímpar) da série acima;

- Encontrar o polo positivo principal e o resíduo do aproximante de Padé;

- Repetir os passos acima até que $\alpha_{c}$ e $\beta$ convirjam para a acurácia desejada. O programa deve guardar os valores dos coeficientes calculados em todos as etapas até o final do programa.

Para diminuir a quantidade de memória utilizada nas últimas ordens do cálculo, escolhemos sempre variáveis ligadas a aglomerados delimitados por sítios vazios, tais como $P_{0}, P_{00}, P_{000}$ e $P_{010}$, uma vez que a expansão em série destas variáveis possui menos termos que as de variáveis delimitadas por sítios ocupados. Escolhemos calcular os aproximantes de Padé de ordem $[n / 2, n / 2]$, de modo que precisamos avançar duas ordens no cálculo da série de $\rho$ para cada nova estimativa de $\alpha_{c}$ e $\beta$. Os valores dos coeficientes $c_{n}^{(1)}, 0 \leq n \leq 21$, da expansão em série de $\rho$ aparecem na tabela 2.2, enquanto as sucessivas estimativas de $\alpha_{c}$ e $\beta$ aparecem na tabela 2.3.

Analisando a tabela 2.2, vemos que o módulo dos coeficientes de $\rho$ aumenta com a ordem dos cálculos. Este fato é estatisticamente esperado, uma vez que o coeficiente $c_{n}^{(1)}$ depende linearmente de todos os coeficientes anteriormente calculados e,

Tabela 2.2: Coeficientes $c_{n}^{(1)}$ da expansão de $\rho$ em série de potências de $\alpha$.

\begin{tabular}{|c|c||c|c|}
\hline$n$ & $c_{n}^{(1)}$ & $n$ & $c_{n}^{(1)}$ \\
\hline 0 & $1,000000000000 \mathrm{E}+00$ & 11 & $-5,074116017903 \mathrm{E}+03$ \\
1 & $-1,000000000000 \mathrm{E}+00$ & 12 & $-1,494581753539 \mathrm{E}+04$ \\
2 & $-1,000000000000 \mathrm{E}+00$ & 13 & $-4,447736306471 \mathrm{E}+04$ \\
3 & $-2,000000000000 \mathrm{E}+00$ & 14 & $-1,332435717211 \mathrm{E}+05$ \\
4 & $-4,500000000000 \mathrm{E}+00$ & 15 & $-4,022276206148 \mathrm{E}+05$ \\
5 & $-1,100000000000 \mathrm{E}+01$ & 16 & $-1,220343327302 \mathrm{E}+06$ \\
6 & $-2,862500000000 \mathrm{E}+01$ & 17 & $-3,723196360385 \mathrm{E}+06$ \\
7 & $-7,708593750000 \mathrm{E}+01$ & 18 & $-1,140871210578 \mathrm{E}+07$ \\
8 & $-2,137216796875 \mathrm{E}+02$ & 19 & $-3,509071017041 \mathrm{E}+07$ \\
9 & $-6,049109971788 \mathrm{E}+02$ & 20 & $-1,083954944686 \mathrm{E}+08$ \\
10 & $-1,739640020677 \mathrm{E}+03$ & 21 & $-3,355197890181 \mathrm{E}+08$ \\
\hline
\end{tabular}


Tabela 2.3: Taxa crítica $\alpha_{c}$ e expoente crítico $\beta$ calculados como o polo principal e o resíduo do aproximante de Padé de ordem $[N, N]$.

\begin{tabular}{|c|c|c|}
\hline$N$ & $\alpha_{c}$ & $\beta$ \\
\hline 1 & 0,300000000 & 0,270000000 \\
2 & 0,304805898 & 0,285825552 \\
3 & 0,303524640 & 0,279691058 \\
4 & 0,304036211 & 0,278055440 \\
5 & 0,303267061 & 0,277588812 \\
6 & 0,303255563 & 0,277431003 \\
7 & 0,303226021 & 0,276820766 \\
8 & 0,303230358 & 0,276932713 \\
9 & 0,303228456 & 0,276881072 \\
10 & 0,303228516 & 0,276882801 \\
\hline
\end{tabular}

conforme $n$ aumenta, torna-se cada vez menos provável que termos de sinais opostos se cancelem inteiramente. Assim, é preciso tomar cuidado ao escrever o programa, de modo que eventuais erros de arredondamento e overflow sejam evitados, ou ao menos detectados.

Utilizando o método de expansão em série perturbativa, Jensen e Dickman [7] obtiveram 25 termos da série supercrítica para a probabilidade de sobrevivência do modelo de contato, cujo comportamento crítico é idêntico ao de $\rho$. Comparando os coeficientes das duas séries até a $21^{\mathrm{a}}$ ordem, vemos que eles coincidem. Analisando a tabela 2.3, vemos que $\alpha_{c}$ e $\beta$ obtidos por aproximantes de Padé convergem muito rapidamente para os valores $\alpha_{c} \cong 0,30323$ e $\beta \cong 0,2769$, que são compatíveis com os resultados obtidos por Jensen e Dickman.

O método exposto neste capítulo pode ser adaptado para o cálculo de outros expoentes críticos estáticos, tais como. Entratnto, não é possível calcular expoentes críticos dinâmicos, como $\nu_{\|}$(ligado ao decaimento algébrico da correlação temporal no ponto crítico), uma vez que este método pressupõe que o sistema já se encontra no estado estacionário, pois todas as derivadas temporais são igualadas a zero. 


\section{Capítulo 3}

\section{O modelo de pilha de areia assimétrico}

Neste capítulo, vamos estudar o modelo de pilha de areia assimétrico (PAA), uma versão do modelo de pilha de areia com restrição de altura que apresenta taxas de transição assimétricas espacialmente. Modelos de pilha de areia têm sido estudados nos últimos anos em parte devido a suas conexões com o conceito de criticalidade auto-organizada, em parte devido a suas próprias características críticas.

\subsection{Criticalidade auto-organizada e modelos de pilha de areia}

O conceito de criticalidade auto-organizada (mais conhecido pela sigla SOC - selforganized criticality) surgiu graças ao trabalho de Bak, Tang e Wiesenfeld [8], Dhar [9] e outros. Ele representa a noção de que um sistema físico pode apresentar naturalmente fenômenos críticos (tais como leis de potência associadas a várias grandezas físicas), sem a intervenção de um agente externo que ajuste algum parâmetro de controle do sistema.

São exemplos de sistemas que apresentam SOC modelos de pilha de areia, nos quais ocorre eliminação de partículas pela fronteira, enquanto a adsorção e difusão de partículas ocorrem em toda a extensão do sistema, sendo que a adsorção deve ocorrer a uma taxa muito menor que os demais processos. Nestas circunstâncias, o sistema é conduzido naturalmente para a criticalidade: a densidade de partículas no sistema varia, devido aos processos de adsorção e eliminação, até que o sistema se encontre 
num estado descrito por leis de potência: uma partícula adsorvida pode provocar uma avalanche (sequência de movimentos de difusão de partículas) que culmina com um novo equilíbrio, seja por remanejamento das posições das demais partículas, seja por eliminação pela fronteira. Grandezas associadas a essas avalanches, tais como o número de sítios atingidos ou o tempo necessário para que ela se extingua, obedecem a leis de potência.

Os trabalhos de Vespignani e Zapperi [11], Dickman, Vespignani e Zapperi [24], entre outros, introduzem uma relação entre modelos de pilha de areia, que apresentam SOC, e modelos com as mesmas regras internas de transição (difusão de partículas a partir de sítios que possuem um número mínimo de partículas) que, no entanto, não apresentam adsorção ou eliminação de partículas, de modo que o número total de partículas é conservado. Estes modelos, conhecidos como fixedenergy sandpiles (FES) devido à interpretação física do número total de partículas como uma medida da energia acumulada no sistema, apresentam estados absorventes, ou seja, configurações nas quais nenhum sítio apresenta o número mínimo de partículas necessário para que ocorra difusão. A densidade crítica dos modelos de pilha de areia é a mesma onde ocorre a transição entre a fase absorvente e a fase estacionária ativa nos modelos FES correspondente.

Em um dos modelos mais conhecidos nesta classe, o modelo estocástico de Manna [10], sítios com 2 ou mais partículas são ativos e, durante um passo de atualização, podem mandar 2 partículas para sítios vizinhos, escolhidos aleatoriamente. Perto do ponto crítico, é observado que os sítios ocupados por mais de 2 partículas são raros, de modo que uma variante do modelo na qual o número máximo de partículas é igual a 2 também exibe o mesmo comportamento crítico.

\subsection{Modelos de pilha de areia com restrição de altura}

Uma variante do modelo de pilha de areia de Manna estudada sob alguns aspectos nos últimos anos $([12,18,25])$ invoca uma restrição quanto ao número máximo de partículas que podem ocupar um sítio da rede. Esta versão é conhecida como modelo de pilha de areia com restrição de altura (devido a uma realização do modelo como uma pilha de areia onde grãos são colocados sobre grãos pré-existentes).

Dickman, Tomé e Oliveira [12] definem este modelo usando as regras a seguir: 
- Numa rede uni ou bidimensional de dimensão linear $L$, colocam-se inicialmente $N<2 L$ partículas, de modo que em nenhum sítio existam mais que duas partículas. A sequência de números de ocupação de cada sítio define uma configuração possível do modelo, enquanto a densidade $\rho=N / L$ é um parâmetro conservdo durante a evolução do sistema.

- Sítios com 2 partículas são ativos, enquanto sítios com 0 ou 1 partícula são inativos. Uma configuração é atualizada escolhendo-se um sítio ativo, que perde suas partículas para seus primeiros vizinhos, contanto que nenhum dos sítios de destino ultrapasse o limite de número de ocupação de 2 partículas. Caso isso ocorra, a partícula permanece no sítio de origem.

- As regras de escolha dos sítios de destino e as taxas de cada movimento definem variantes do modelo. Na referência [12], são estudadas duas regras: a "regra independente", na qual cada partícula é direcionada para um dos sítios de destino com a mesma probabilidade, independentemente do movimento da outra partícula, e a "regra cooperativa", na qual o movimento das duas partículas é coordenado de modo que o sítio central perca sempre o número máximo de partículas possível, sem desrespeitar o limite de altura.

Dantas e Stilck [25] definem uma variante do modelo de pilha de areia com restrição de altura (2 partículas) em uma dimensão que depende de um parâmetro contínuo $\lambda$. Com probabilidade $(1+\lambda) / 2$, as partículas seguem para sítios distintos, enquanto elas vão para o mesmo sítio com probabilidade $(1-\lambda) / 2$. Quando $\lambda=$ 0, obtemos novamente o modelo de Dickman, Tomé e Oliveira, utilizando a regra independente.

Todos os modelos acima apresentam uma transição de fase, que ocorre quando a densidade de partículas $\rho$ assume um valor crítico $\rho_{c}$, separando a fase estacionária ativa $\left(\rho>\rho_{c}\right)$, onde o número de sítios ativos é não-nulo, e a fase absorvente $\left(\rho<\rho_{c}\right)$, que consiste de uma infinidade (no limite termodinâmico $L, N \rightarrow \infty$ ) de estados absorventes, onde não existem sítios ativos. A exceção a esse comportamento é o modelo de Dantas e Stilck para $\lambda=-1$, no qual o número de sítios ativos é uma grandeza conservada durante a evolução temporal, e não existe uma transição de fase. As transições de fase de cada modelo são determinadas utilizando-se aproximações de campo médio e simulações.

Em todos os modelos acima mencionados, observa-se uma pequena alteração no valor da densidade crítica em relação ao valor obtido para versões do modelo 
sem restrição de altura. Em particular, para o modelo de Dickman, Tomé e Oliveira unidimensional, na regra independente, $\rho_{c}=0,929780(7)$ (valor atualizado por Dickman [18]), enquanto na versão sem restrição de altura correspondente, $\rho_{c}=0,9488$ $[12]$.

De acordo com simulações, o expoente crítico $\beta$, relacionado ao comportamento do parâmetro de ordem (densidade de sítios ativos) quando $\rho$ se aproxima do valor crítico, é igual a 0,289(12) na referência [18], mas varia entre 0,4 e 0,6 com o parâmetro $\lambda$ no modelo de Dantas e Stilck [25]. O expoente $\nu_{\perp}$, relacionado com a divergência do comprimento de correlação à medida que $\rho$ se aproxima do ponto crítico, é dado como 1,355(18) em [18], e varia entre 1,85 e 0,9 (no limite $\lambda \rightarrow-1$ ) em [25]. O expoente $\nu_{\|}$, relacionado à divergência do tempo de correlação perto do ponto crítico, vale 2,03(8), a partir de [18]. Podemos ver que um estudo cuidadoso do modelo de pilha de areia com restrição de altura e de algumas de suas variantes pode contribuir para o melhor entendimento dessa importante classe de modelos estocásticos.

\subsection{O modelo de pilha de areia assimétrico I - Generalidades}

No restante deste capítulo, vamos estudar uma nova versão do modelo de pilha de areia com restrição de altura, cuja taxa de transição não possui simetria espacial: em média, mais partículas movimentam-se para um lado do que para o outro, dependendo do valor de um parâmetro $p$. Podemos entender este novo modelo como a idealização de uma pilha de areia rasa cuja base é inclinada de um certo ângulo $\theta$ em relação à horizontal. Assim, partículas que estão em picos locais tendem a escorregar mais para um lado do que para o outro. Ignoramos o comportamento das extremidades, onde deve acontecer uma diminuição (na extremidade mais alta) ou o aumento do número de grãos de areia, e concentramos nosso estudo na seção central da pilha, onde o sistema deve se estabilizar de forma a apresentar um fluxo constante de partículas para baixo.

Consideramos uma rede unidimensional com $L$ sítios e $N$ partículas. Cada sítio pode estar vazio ou ocupado por no máximo 2 partículas: uma configuração é caracterizada pela sequência de números de ocupação de cada sítio na rede. Por definição, um sítio ocupado por 2 partículas é um sítio ativo. Impomos condições periódicas 
Tabela 3.1: Probabilidades de transição para todas as trincas compostas por um sítio central ativo e ao menos um sítio de destino inativo.

\begin{tabular}{|c|c||c|c|}
\hline Transição & Taxa & Transição & Taxa \\
\hline $020 \rightarrow 002$ & $(1-p)^{2}$ & $120 \rightarrow 102$ & $(1-p)^{2}$ \\
$020 \rightarrow 101$ & $2 p(1-p)$ & $120 \rightarrow 201$ & $2 p(1-p)$ \\
$020 \rightarrow 200$ & $p^{2}$ & $120 \rightarrow 210$ & $p^{2}$ \\
\hline $021 \rightarrow 012$ & $(1-p)^{2}$ & $121 \rightarrow 112$ & $(1-p)^{2}$ \\
$021 \rightarrow 102$ & $2 p(1-p)$ & $121 \rightarrow 202$ & $2 p(1-p)$ \\
$021 \rightarrow 201$ & $p^{2}$ & $121 \rightarrow 211$ & $p^{2}$ \\
\hline $022 \rightarrow 112$ & $2 p(1-p)$ & $220 \rightarrow 202$ & $(1-p)^{2}$ \\
$022 \rightarrow 202$ & $p^{2}$ & $220 \rightarrow 211$ & $2 p(1-p)$ \\
\hline $122 \rightarrow 212$ & $p(2-p)$ & $221 \rightarrow 212$ & $1-p^{2}$ \\
\hline
\end{tabular}

de contorno, de modo que os sítios $N$ e 1 são vizinhos. Definimos um parâmetro $p$, que controla o fluxo de partículas na rede, no intervalo $0 \leq p \leq 1$. Para $p=0,5$, este modelo é idêntico ao modelo apresentado por Dickman, Tomé e Oliveira [12] seguindo a regra independente. Estamos interessados em estudar como o ponto crítico e alguns expoentes críticos variam quando tomamos $p \neq 0,5$.

Atualizamos uma configuração por meio das regras a seguir:

- Escolhemos um sítio ao acaso. Se este sítio não for ativo, a tentativa de atualização não ocorre.

- Se o sítio escolhido for ativo, escolhemos um sítio de destino para cada uma das partículas, independentemente, com as probabilidades: $p$, para o sítio à esquerda mais próximo, e $1-p$, para o sítio à direita mais próximo.

- Os movimentos escolhidos são realizados, desde que os sítios de destino fiquem, no máximo, com 2 partículas. Se qualquer um dos movimentos não for possível, a partícula correspondente permanece no sítio de origem.

- Os passos acima são repetidos um número fixo de vezes.

Como o número de partículas não muda durante um passo de atualização, a densidade de partículas $\rho=N / L$ é um parâmetro útil para classificar configurações. O conjunto de regras acima permite escrever a tabela 3.1, com todas as transições possíveis envolvendo três sítios vizinhos: o sítio central ativo e os dois possíveis sítios de destino. 
Da tabela 3.1, podemos ver que uma configuração é ativa (ou seja, é possível alcançar outras configurações a partir dela) se existe ao menos um sítio ativo cercado por ao menos um sítio inativo. Assim, para que uma configuração não seja ativa, é necessário que não haja sítios ativos, ou que não haja sítios inativos.

Para que exista ao menos uma configuração inativa, para um certo valor de $\rho$, é necessário que $\rho \leq 1$. No limite $\rho=1$, a única configuração inativa posssível é aquela onde todos os sítios possuem uma partícula, uma vez que todas as outras configurações possíveis necessariamente possuem ao menos um sítio ativo.

No restante, deste capítulo, vamos designar por $P_{x}$ a probabilidade de que uma sequência $x$ de números de ocupação seja encontrada. Assim, $P_{2}$ é a probabilidade de que um sítio esteja ocupado por 2 partículas, enquanto $P_{020}$ é a probabilidade de que, escolhendo-se três sítios vizinhos, o sítio central esteja ocupado por duas partículas, enquanto os sítios à direita e à esquerda estejam vazios.

De modo geral, podemos prever que, quando $\rho$ é muito pequena, a densidade de sítios ativos $P_{2}$ evolui no tempo para zero, uma vez que, segundo a tabela 3.1, existe uma transição que anula o número de sítios ativos na trinca $(020 \rightarrow 101)$, a qual deve ocorrer com mais frequência quando o sistema está no regime de baixa densidade. No regime de alta densidade, o número de sítios ativos deve ser diferente de zero. Assim, no regime de baixa densidade, o sistema evolui até alcançar um estado absorvente (que corresponde a uma configuração inativa) e é congelado, sem chances de evolução futura.

Como não existem configurações inativas quando $\rho>1$, o sistema deve passar por uma transição de fase entre uma fase absorvente e uma fase estacionária ativa quando a densidade atinge um valor crítico $\rho_{c}$, o qual pode depender do parâmetro $p$. Para caracterizar a transição entre as duas fases, introduzimos um parâmetro de ordem [16] que se anula na fase absorvente e é diferente de zero na fase ativa. Uma escolha natural para o parâmetro de ordem é a densidade de sítios ativos $P_{2}$.

A única configuração que não apresenta sítios inativos corresponde à densidade máxima de partículas $\rho=2$. Não é apropriado denominar este estado como um estado absorvente, uma vez que não existem outras configurações que possam evoluir no tempo de modo a alcançar tal configuração.

Como primeiro passo para estudar o comportamento deste modelo, vamos escrever a equação de evolução temporal para a densidade de sítios ativos. De modo geral, a equação de evolução temporal de qualquer grandeza dada como uma média 
sobre todas as configurações do sistema

$$
F=\sum_{\sigma} F(\sigma) P(\sigma, t)
$$

pode ser obtida a partir da equação mestra [5], que na sua forma mais geral é dada por:

$$
\frac{d}{d t} P(\sigma, t)=\sum_{\sigma^{\prime}}\left\{W\left(\sigma, \sigma^{\prime}\right) P\left(\sigma^{\prime}, t\right)-W\left(\sigma^{\prime}, \sigma\right) P(\sigma, t)\right\}
$$

onde $P(\sigma, t)$ é a probabilidade de se encontrar o sistema na configuração $\sigma$ no instante $t$, e $W\left(\sigma^{\prime}, \sigma\right)$ é a taxa de transição da configuração $\sigma$ para a configuração $\sigma^{\prime}$. Em nosso problema, a taxa de transição entre duas configurações $\sigma$ e $\sigma^{\prime}$ é não-nula apenas se as duas configurações diferem por, no máximo, três sítios vizinhos.

Seja $P_{x y z}$ a probabilidade da sequência de números de ocupação $x y z$, para quaisquer valores de $x, y$ e $z$. Consultando a tabela 3.1, obtemos:

$$
\frac{d}{d t} P_{2}=2 p(1-p)\left(P_{121}-P_{020}-P_{022}-P_{220}\right)
$$

Como é comum no estudo de modelos em física estatística fora do equilíbrio, a equação para a evolução temporal de $P_{2}$ envolve outras grandezas. As equações de evolução temporal para estas grandezas, como veremos na seção 3.4, envolvem ainda outras grandezas, de modo que encontramos uma hierarquia de equações diferenciais acopladas, a qual deve ser truncada em algum ponto para que possamos estudar o seu comportamento, estudo feito na seção 3.4.

Analisando a equação acima, vemos que, quando $p=0$ ou 1 , a densidade de sítios ativos mantém-se constante, para qualquer configuração inicial, enquanto para $0<p<1$, a densidade de sítios ativos pode variar, dando origem a uma transição de fase quando $\rho=\rho_{c}$. Um estudo mais aprofundado do primeiro caso é feito a seguir.

\subsubsection{Estudo do modelo para $p=0$ ou 1}

Como foi notado estudando a equação (3.3), quando o parâmetro $p$ assume os valores 0 ou 1, a densidade de sítios ativos mantém-se constante, independentemente da configuração inicial. Analisando mais a fundo esta situação, vemos primeiramente que os casos $p=0$ e $p=1$ são de fato simétricos. Para $p=0$, o único movimento 
Tabela 3.2: Taxas de transição para todas as trincas ativas, quando $p=0$.

\begin{tabular}{|c|c||c|c|}
\hline Transição & Taxa & Transição & Taxa \\
\hline $020 \rightarrow 002$ & 1 & $021 \rightarrow 012$ & 1 \\
$120 \rightarrow 102$ & 1 & $121 \rightarrow 112$ & 1 \\
$220 \rightarrow 202$ & 1 & $221 \rightarrow 212$ & 1 \\
\hline
\end{tabular}

Tabela 3.3: Simplificação das taxas de transição da tabela 3.2.

\begin{tabular}{|c|c|}
\hline Transição & Taxa \\
\hline $20 \rightarrow 02$ & 1 \\
$21 \rightarrow 12$ & 1 \\
\hline
\end{tabular}

permitido de uma partícula é para a direita, enquanto para $p=1$, permite-se apenas o movimento para a esquerda. Para as discussões a seguir, vamos assumir $p=0$. Os resultados obtidos podem ser facilmente convertidos para a situação $p=1$.

Se $p=0$, as únicas transições possíveis aparecem na tabela 3.2. Vemos que as taxas de transição não dependem do número de partículas do sítio à esquerda do sítio ativo, de modo que a informação contida na tabela 3.2 pode ser condensada para a tabela 3.3.

Observando a tabela 3.3, notamos que o número de sítios com 0 e 1 partícula também não muda conforme o sistema evolui, porque as duas transições possíveis conservam o número de sítios com qualquer número de partículas. De fato, $P_{0}$ e $P_{1}$ são unicamente determinados a partir de $P_{2}$ e $\rho$ (ver capítulo 3.4).

$\mathrm{Na}$ transição $21 \rightarrow 12$, uma das partículas do sítio ativo permanece no lugar, enquanto a outra encontra-se com a partícula residente no sítio à direita, transformando um sítio inativo em um sítio ativo. Entretanto, supondo que as partículas no modelo de pilha de areia assimétrico são indistinguíveis, podemos considerar que ocorre uma permuta total de partículas entre os dois sítios para construir a configuração resultante da transição: as partículas do sítio ativo movem-se para a direita e a partícula inativa "move-se" para a esquerda. Do mesmo modo, a transição $20 \rightarrow 02$ pode ser descrita como uma permuta entre o sítio ativo e o sítio inativo. Assim, se consideramos que um sítio ativo corresponde a um tipo de "partículas" e os sítios inativos correspondem a outros dois tipos de "partículas", então cada configuração do modelo de pilha de areia (ou seja, cada sequência possível de números de ocupação) corresponde univocamente a uma configuração de um modelo com três 


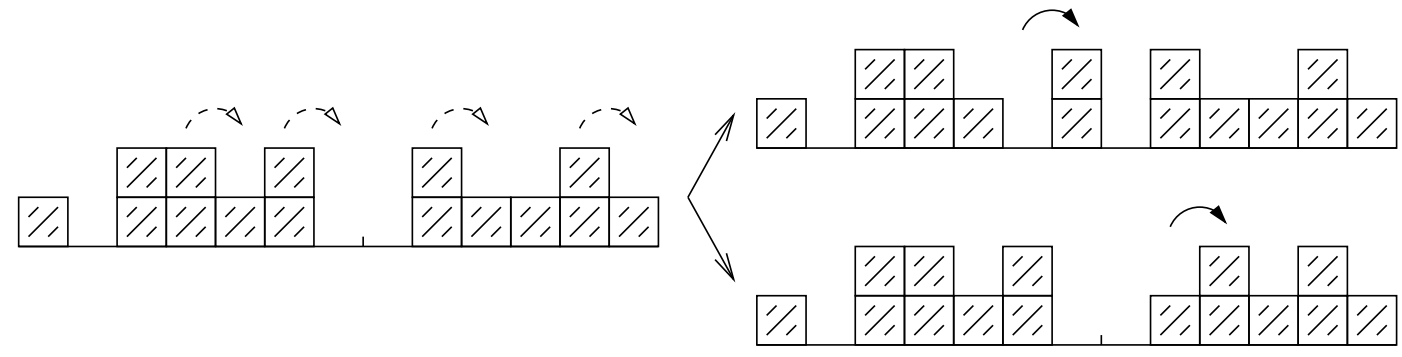

(a) Partículas indistinguíveis

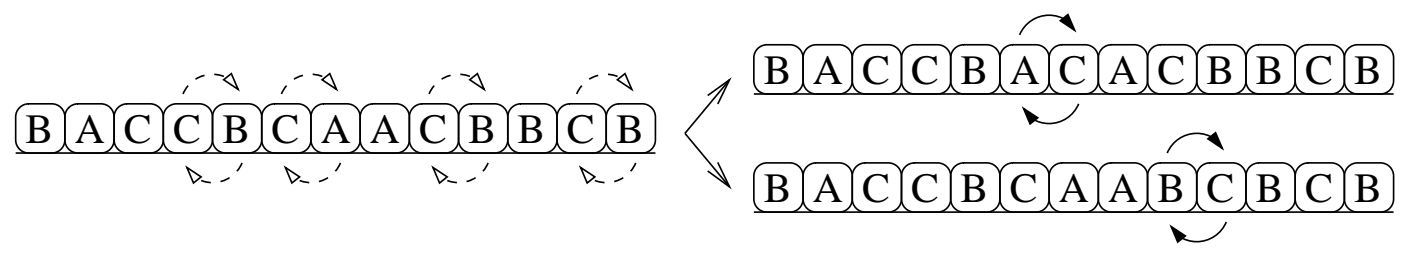

(b) Partículas $A, B$ e $C$

Figura 3.1: À esquerda, ilustração de transições possíveis quando $p=0$; à direita, duas possíveis configurações resultantes. As configurações mostradas na representação de partículas $A, B$ e $C$ são as mesmas mostradas na representação de partículas indistinguíveis.

tipos de partículas: $A, B$ e $C$, que evolui de acordo com as regras abaixo:

- Cada sítio é ocupado por uma das partículas $A, B$ ou $C$. Não existem sítios vazios ou sítios com mais de uma partícula.

- Partículas $A$ e $B$ movem-se para a esquerda, enquanto partículas $C$ movem-se para a direita, um sítio por vez.

- Um passo de atualização ocorre quando uma partícula $C$ e uma partícula $A$ ou $B$ trocam de lugar, desde que as partículas movam-se na direção apropriada. Esse movimento ocorre com taxa unitária.

Na figura 3.1, ilustramos a correspondência entre as duas representações do modelo de pilha de areia.

Podemos ver claramente que este sistema não apresenta uma transição dinâmica para um estado absorvente. Como visto, o número de partículas $A, B$ e $C$ permanece constante. Assim, basta que exista ao menos uma partícula $C$ e ao menos uma das partículas $A$ ou $B$ para que exista ao menos um par $C A$ ou $C B$, e o sistema pode deixar a configuração atual. 
Também podemos constatar que partículas $A$ e $B$ apresentam sempre a mesma ordem relativa (se escolhermos a posição de uma partícula específica $A$ ou $B$ como a origem), uma vez que as transições possíveis no modelo sempre envolvem uma única partícula $A$ ou $B$. De fato, se numerarmos as partículas $A$ e $B$ na ordem em que elas aparecem na condição inicial (desprezando partículas $C$ ), é possível mudar essa numeração apenas quando uma partícula $A$ ou $B$ no primeiro sítio da rede troca de lugar com uma partícula $C$ no último sítio da rede (uma vez que impomos condições de contorno periódicas). Assim, a ordem relativa de partículas $A$ e $B$ está definida em uma configuração se fornecermos a ordem inicial e a posição inicial da partícula que ocupa, no momento inicial, a primeira posição.

Consideremos agora o espaço de configurações associado ao modelo de pilha de areia, caracterizado pelo tamanho $L$ da rede e pelo número $N$ de partículas. Para $p=0$, esse espaço reparte-se em subespaços tais que a trajetória do sistema no espaço de configurações se restringe ao subespaço onde a configurações inicial está localizada. Cada subespaço é caracterizado pela sequência de partículas $A$ e $B$ (ou seja, pela sequência de sítios inativos do modelo de pilha de areia), enquanto cada ponto dentro do subespaço é caracterizado pela posição das partículas $C$ dentro da sequência de partìculas $A$ e $B$ (ou seja, pela distribuição de sítios ativos no modelo de pilha de areia).

Para caracterizar completamente uma configuração, além da ordem relativa das partículas $A$ e $B$, precisamos das posições de cada partícula. Conhecendo-se estas posições, a posição das partículas $C$ fica determinada por exclusão. Assim, a evolução temporal de uma configuração é determinada pelos movimentos das partículas $A$ e $B$ : cada transição possível corresponde ao salto de uma das partículas $A$ ou $B$ para o primeiro sítio à esquerda.

Usando esta representação do modelo, podemos concluir que cada subespaço caracterizado por uma ordem de partículas $A$ e $B$ é ergódico, portanto o sistema está em equilíbrio quando $p=0$ ou 1 . De fato, como cada configuração é completamente caracterizada pelas posições das partículas $A$ e $B$, e como a ordem relativa entre estas partículas não muda, para passar de uma configuração I para uma configuração II, basta ajustar as posições das partículas $A$ e $B$ uma por vez. Cada passo de atualização ajusta a posição de uma partícula $A$ ou $B$ de uma posição. Assim, calculando a distância entre a posição de cada partícula na configuração I e na configuração II, obtemos o número de passos necessário para ir de uma configuração à outra. 


\subsubsection{Fluxo de partículas}

No modelo de pilha de areia assimétrico, uma vez que associamos probabilidades diferentes para o movimento de uma partícula ativa nos dois sentidos (maior para a direita se $0 \leq p<0,5$, e maior para a esquerda se $0,5<p \leq 1$ ). existe um fluxo de partículas na rede, mesmo após o sistema ter alcançado o estado estacionário.

Definimos a variável local $\phi_{i}(t)$ como o número de partículas que sai do sítio $i$ e se desloca no sentido positivo (em direção ao sítio $i+1$ ), menos o número de partículas que sai do mesmo sítio e se desloca no sentido oposto, em uma unidade de tempo. Assim, se o sítio $i$ for ativo no instante $t$, a variável $\phi_{i}(t)$ pode assumir os valores $0, \pm 1, \pm 2$. Se o sítio $i$ for inativo no instante $t, \phi_{i}(t)=0$. $\phi_{i}(t)$ é uma variável estocástica, e podemos obter informações mais úteis a partir de seu valor médio no intervalo de tempo $\Delta t$, o qual é menos suscetível a flutuações estocásticas.

Conforme o sistema evolui até alcançar o estado estacionário ativo, $\phi_{i}$ deve assumir um valor constante (não-nulo) e homogêneo $\phi$. O fluxo estacionário $\phi$ pode ser calculado como uma média sobre todos os sítios: $\phi$ é igual ao número de partículas que se movimentam para a direita (não importando o sítio de origem) menos o número de partículas que se deslocam para a esquerda em uma unidade de tempo, dividido pelo tamanho da rede $L$.

Tomando como unidade de tempo $N$ tentativas de atualização, como é comum em modelos estocásticos, podemos exprimir $\phi$ em função das variáveis densidades de trincas no limite $N \rightarrow \infty$. Consideramos, como exemplo, uma trinca de sítios 020 presente na rede. De acordo com a tabela 3.1, se o sítio central for escolhido para a transição, as duas partículas irão para a esquerda com probabilidade $p^{2}$, para a direita com probabilidade $(1-p)^{2}$, e para lados distintos com probabilidade $2 p(1-p)$. No limite $N \rightarrow \infty$, o sítio central deve ser escolhido em média uma vez por unidade de tempo, e a contribuição desse sítio para o cálculo de fluxo é igual a:

$$
\phi_{i}= \begin{cases}-2, & \text { com probabilidade } p^{2} \\ 0, & \text { com probabilidade } 2 p(1-p) \\ +2, & \text { com probabilidade }(1-p)^{2}\end{cases}
$$

Somando sobre todos os sítios ativos com dois vizinhos vazios e dividindo por $L$, obtemos:

$$
\phi_{020}=\left\{-2 p^{2}+2(1-p)^{2}\right\} P_{020}=(2-4 p) P_{020}
$$




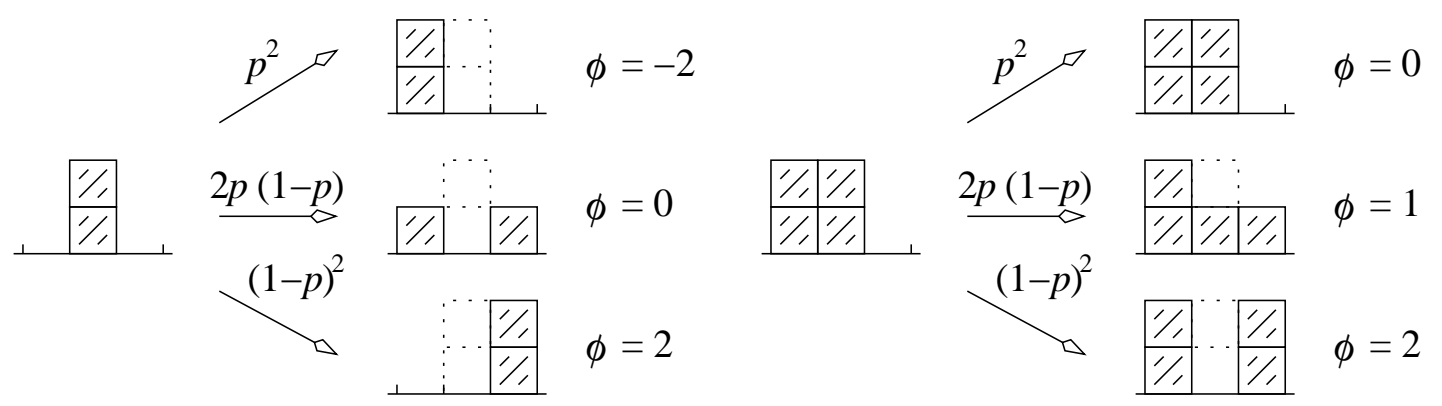

Figura 3.2: Cálculo do fluxo para as configurações de trinca 020 e 220.

O fluxo total é obtido somando sobre todas as trincas cujo sítio central é ativo. Notamos que, para cada configuração de trinca, o coeficiente numérico é diferente, uma vez que movimentos tais que o sítio de destino é ocupado por mais de duas partículas não ocorrem, e a partícula permanece no sítio de origem (como mostra a figura 3.2).

Em termo das densidades de trincas, o fluxo total é dado por:

$$
\begin{aligned}
\phi & =\phi_{020}+\phi_{021}+\phi_{022}+\phi_{120}+\phi_{121}+\phi_{122}+\phi_{220}+\phi_{221} \\
& =(2-4 p) P_{020}+\left(1-2 p-p^{2}\right) P_{021}-2 p P_{022}+\left(2-4 p+p^{2}\right) P_{120}+ \\
& +(1-2 p) P_{121}+\left(-2 p+p^{2}\right) P_{122}+(2-2 p) P_{220}+\left(1-p^{2}\right) P_{221}
\end{aligned}
$$

Podemos simplificar a expressão acima. Uma vez que as partículas de um sítio ativo movem-se de forma independente, deve ser possível expressar o fluxo como uma função das densidades de pares de partículas. Valem as seguintes condições de normalização:

$$
\begin{aligned}
& P_{022}=P_{02}-P_{020}-P_{021} \\
& P_{122}=P_{12}-P_{120}-P_{121} \\
& P_{220}=P_{20}-P_{020}-P_{120} \\
& P_{221}=P_{21}-P_{021}-P_{121}
\end{aligned}
$$

Substituindo (3.7), (3.8), (3.9) e (3.10) em (3.6), obtemos a expressão a seguir:

$$
\phi=-2 p P_{02}-\left(2 p-p^{2}\right) P_{12}+(2-2 p) P_{20}+\left(1-p^{2}\right) P_{21}
$$


A equação (3.11) pode ser reescrita de várias formas. Uma forma que se mostrará útil quando calcularmos valores numéricos para $\phi$ consiste em utilizarmos as condições de normalização:

$$
\begin{aligned}
1 & =P_{0}+P_{1}+P_{2} \\
\rho & =P_{1}+2 P_{2} \\
P_{0} & =P_{00}+P_{01}+P_{02}=P_{00}+P_{10}+P_{20} \\
P_{1} & =P_{10}+P_{11}+P_{12}=P_{01}+P_{11}+P_{21} \\
P_{2} & =P_{20}+P_{21}+P_{22}=P_{02}+P_{12}+P_{22}
\end{aligned}
$$

para escrever $\phi$ em função das variáveis independentes $P_{0}, P_{00}, P_{01}, P_{10}$ e $P_{11}$ (por coincidência, $\phi$ é independente de $P_{0}$ ):

$$
\phi=(1-2 p)\left(2-\rho-2 P_{00}-P_{01}-2 P_{10}-P_{11}\right)+p^{2}\left(P_{01}-P_{10}\right)
$$

Também podemos reescrever (3.11) de modo a ficar evidente que $\phi$ satisfaz a duas propriedades básicas:

- Simetria de inversão espacial: $\phi$ deve mudar de sinal se invertemos a ordem dos sítios e simultaneamente mudamos o valor de $p$ para $1-p$;

- O fluxo deve se anular no estado absorvente (e quando $\rho=2$, mas este fato é trivial).

Utilizando as condições (3.16), eliminamos $P_{12}$ e $P_{21}$ da equação (3.11), obtendo:

$$
\phi=(1-2 p)\left(P_{2}-P_{22}\right)+(1-p)^{2} P_{20}-p^{2} P_{02}
$$

Vemos agora que, se invertemos a ordem dos sítios e trocarmos $p$ por $1-p$, obtemos um fluxo com o mesmo módulo e sinal oposto.

\subsection{O modelo de pilha de areia assimétrico II - Aproximações de campo médio}

A seguir, vamos estudar o modelo de pilha de areia assimétrico no intervalo $0<p<$ 1, de forma analítica. Vamos obter estimativas para a dependência da densidade 
crítica e do fluxo de partículas por meio de aproximações de campo médio.

\subsubsection{Aproximação de campo médio simples}

A aproximação de campo médio mais básica é a de campo médio simples, que consiste em supor que a probabilidade de se encontrar o sítio $i$ em um certo estado de ocupação é independente dos estados de ocupação dos vizinhos. Assim, todas as densidades de sequências de dois ou mais sítios são aproximadas pelo produto das densidades de sítio único correspondentes:

$$
P_{x y \ldots}=P_{x} P_{y} \ldots, \quad x, y, \ldots=0,1 \text { ou } 2
$$

Com esta aproximação, a equação (3.3) toma a forma:

$$
\frac{d}{d t} P_{2}=2 p(1-p)\left(P_{1}^{2} P_{2}-P_{0}^{2} P_{2}-2 P_{0} P_{2}^{2}\right)
$$

Não precisamos escrever as equações de evolução temporal para as variáveis $P_{0}$ e $P_{1}$, que aparecem acima, porque elas não são independentes de $P_{2}$. Utilizando (3.12) e (3.13), podemos escrever:

$$
\begin{aligned}
& P_{0}=1-\rho+P_{2} \\
& P_{1}=\rho-2 P_{2}
\end{aligned}
$$

Substituindo (3.21) e (3.22) em (3.20), obtemos:

$$
\frac{d}{d t} P_{2}=2 p(1-p)\left[(2 \rho-1) P_{2}-4 P_{2}^{2}+P_{2}^{3}\right]
$$

Para obtermos $P_{2}$ no estado estacionário, igualamos a equação acima a zero, obtendo as raízes:

$$
P_{2}=0,2 \pm \sqrt{5-2 \rho}
$$

Desprezamos a solução $P_{2}=2+\sqrt{5-2 \rho}$, porque ela não satisfaz a condição $P_{2} \leq 1$. A solução com sinal negativo é aceitável, desde que:

$$
0 \leq 2-\sqrt{5-2 \rho} \leq 1 \rightarrow 0,5 \leq \rho \leq 2
$$

A solução $P_{2}=0$ é aceitável na região de baixa densidade, mas deixa de ser possível 


\subsection{O MODELO PAA II - APROXIMAÇÕES DE CAMPO MÉDIO}

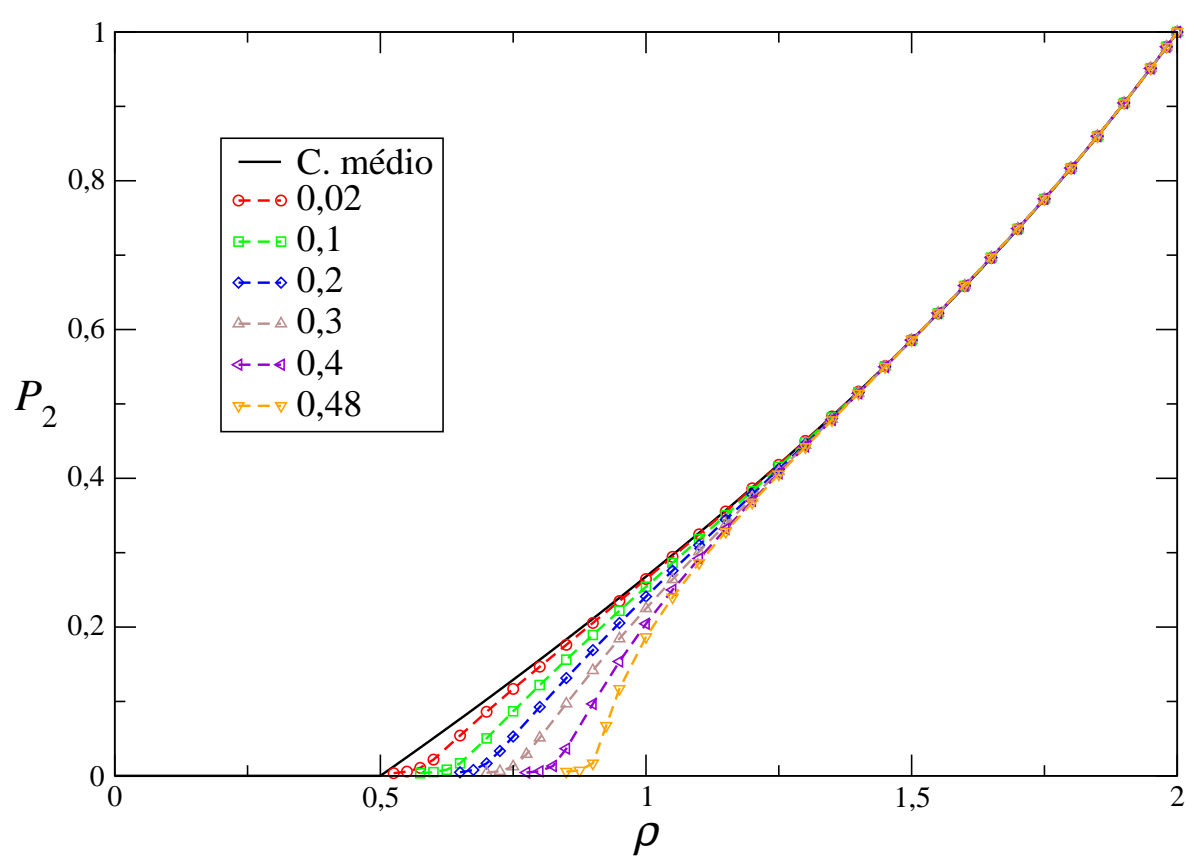

Figura 3.3: Densidade de sítios ativos $P_{2}$ obtida por aproximação de campo médio simples (linha contínua) e por simulação do modelo para diversos valores de $p$, indicados na legenda (linhas tracejadas). O tamanho da rede para a simulação foi fixado em 1000 sítios.

quando $\rho>1$, de modo que devemos ter uma transição de fase em algum ponto dentro da região $0,5 \leq \rho \leq 1$. Impondo que a densidade de sítios ativos seja contínua com $\rho$, como os resultados de simulação implicam, encontramos a densidade crítica $\rho_{c}=0,5$, que separa as fases ativa $\left(\rho>\rho_{c}\right)$ e absorvente $\left(\rho<\rho_{c}\right)$. A densidade de sítios ativos em função da densidade é mostrado na figura 3.3. Na mesma figura, colocamos resultados obtidos por simulação computacional do modelo numa rede de 1000 sítios com condições periódicas de contorno. Vamos discutir em mais detalhes como foi feita a simulação na seção 3.5 .

Analisando essa figura, vemos que, na aproximação de campo médio simples, $p$ não influi no comportamento de $P_{2}$ no estado estacionário, embora seja visível nos resultados de simulação uma dependência de $P_{2} \operatorname{com} p$. De acordo com a equação (3.20), a única influência de $p$ no comportamento de $P_{2}$ é atribuir uma escala temporal ao desaparecimento do comportamento transiente e o estabelecimento do estado estacionário.

Para estudar o comportamento de $P_{2}$ perto do ponto crítico, vamos fazer a 
substituição $\rho=0,5+\rho^{*}$ e considerar o limite $\rho^{*} \rightarrow 0$. Temos:

$$
P_{2}=2-\sqrt{5-2\left(\frac{1}{2}+\rho^{*}\right)}=2-2 \sqrt{1-\frac{\rho^{*}}{2}} \Rightarrow \lim _{\rho^{*} \rightarrow 0} P_{2}=\frac{\rho^{*}}{2}
$$

de modo que $P_{2}$ é linear com $\rho$ perto do ponto crítico. Definindo o expoente crítico $\beta$, ligado ao decaimento do parâmetro de ordem perto do ponto crítico:

$$
P_{2} \approx A\left|\rho-\rho_{c}\right|^{\beta}
$$

vemos que $\beta=1$ na aproximação de campo médio simples.

\subsubsection{Aproximação de campo médio de pares}

Comparando os resultados de simulação e a aproximação de campo médio simples mostrados no gráfico 3.3, vemos que essa aproximação não fornece uma boa estimativa do ponto crítico, não prevendo uma dependência com $p$. Assim, propomos melhorar a nossa aproximação para campo médio de pares.

Esta aproximação consiste em tomar como variáveis todas as densidades de pares de sítios no estado estacionário, além das densidades de sítios únicos. As densidades de sequências com um número maior de sítios são aproximadas por:

$$
P_{x y \ldots z}=\frac{P_{x y} P_{y \ldots z}}{P_{y}}
$$

Para justificar a aproximação acima, consideramos a probabilidade condicional $P(x \mid y \ldots z)$, que é a probabilidade de que o primeiro sítio da sequência seja $x$, dado que os sítios seguintes sejam $y . . z$. Podemos escrever, de acordo com esta definição:

$$
P(x \mid y \ldots z)=\frac{P_{x y \ldots z}}{P_{y \ldots z}}
$$

A aproximação de campo médio de pares consiste em supor que $P(x \mid y \ldots z)$ depende apenas do estado de ocupação do primeiro sítio da sequência $y \ldots z$, ou seja, supor que sítios separados por dois ou mais vizinhos são independentes. Assim, temos:

$$
P(x \mid y \ldots z)=P(x \mid y) \Rightarrow \frac{P_{x y \ldots z}}{P_{y \ldots z}}=\frac{P_{x y}}{P_{y}}
$$

Isolando $P_{x y . . . z}$, obtemos a aproximação (3.28). Aproximações superiores também 
podem ser obtidas a partir deste raciocínio, impondo que $P(x \mid y \ldots z)$ dependa dos estados de ocupação dos primeiros $n$ vizinhos (aproximação de ordem $n+1$ ).

Dentre todas as densidades de pares de sítios possíveis, apenas 4 são independentes: as demais podem ser reescritas como funções das 4 primeiras variáveis, de $P_{2}$ e de $\rho$. Escolhemos como as 4 variáveis: $P_{00}, P_{01}, P_{10}$ e $P_{11}$. Com esta escolha, as demais densidades de pares de sítios são dadas por:

$$
\begin{aligned}
& P_{02}=P_{0}-P_{00}-P_{01}=1-\rho+P_{2}-P_{00}-P_{01} \\
& P_{20}=P_{0}-P_{00}-P_{10}=1-\rho+P_{2}-P_{00}-P_{10} \\
& P_{12}=P_{1}-P_{10}-P_{11}=\rho-2 P_{2}-P_{10}-P_{11} \\
& P_{21}=P_{1}-P_{01}-P_{11}=\rho-2 P_{2}-P_{01}-P_{11} \\
& P_{22}=P_{2}-P_{20}-P_{21}=-1+2 P_{2}+P_{00}+P_{01}+P_{10}+P_{11}
\end{aligned}
$$

As equações de evolução temporal das novas variáveis são obtidas a partir da tabela 3.1:

$$
\begin{aligned}
\frac{d}{d t} P_{00} & =\left(1-2 p+2 p^{2}\right) P_{020}-p(2-p) P_{002}-\left(1-p^{2}\right) P_{200} \\
\frac{d}{d t} P_{01} & =\left(1-2 p+2 p^{2}\right) P_{021}+2 p(1-p)\left(P_{002}+P_{020}+P_{120}\right) \\
& -p(2-p) P_{012}-\left(1-p^{2}\right) P_{201} \\
\frac{d}{d t} P_{10} & =\left(1-2 p+2 p^{2}\right) P_{120}+2 p(1-p)\left(P_{020}+P_{021}+P_{200}\right) \\
& -p(2-p) P_{102}-\left(1-p^{2}\right) P_{210} \\
\frac{d}{d t} P_{11} & =\left(1-2 p+2 p^{2}\right) P_{121}+2 p(1-p)\left(P_{022}+P_{102}+P_{201}+P_{220}\right) \\
& -p(2-p) P_{112}-\left(1-p^{2}\right) P_{211}
\end{aligned}
$$

Efetuando as substituições (3.28), (3.31), (3.32), (3.33), (3.34) e (3.35) nas equações de evolução temporal (3.3), (3.36), (3.37), (3.38) e (3.39), obtemos um conjunto de 5 equações diferenciais não-lineares nas variáveis $P_{2}, P_{00}, P_{01}, P_{10}$ e $P_{11}$, com parâmetros $p$ e $\rho$, as quais são complexas demais para que seja possível encontrar uma forma analítica para cada variável em função de $t, p$ e $\rho$.

Para determinar a dependência com $p$ e $\rho$ deste conjunto de variáveis no estado estacionário, optamos por integrar numericamente as 5 equações obtidas até que cada uma das variáveis $P_{2}, P_{00}, P_{01}, P_{10}$ e $P_{11}$ deixe de variar entre dois passos 
de integração. Definimos as condições iniciais do modelo como uma distribuição aleatória de partículas com densidade $\rho$, respeitando a condição de cada sítio poder ser ocupado por no máximo duas partículas. Efetuamos a integração discretizando cada uma das equações diferenciais pelo método de Euler [26], de modo que:

$$
P_{x x}(t+\Delta t)=P_{x x}(t)+\Delta t \frac{d}{d t} P_{x x}(t)
$$

onde $\Delta t=0,001$. Os intervalos de tempo necessários para que todas as variaeis alcancem os valores estacionários são da ordem de 10 a 100 unidades de tempo (ou seja, entre $10^{4}$ e $10^{5}$ passos de integração). Os resultados obtidos para alguns valores de $p$ são mostrados na figura 3.4 .

Comparando os gráficos 3.4a, 3.4b e 3.4c, é possível observar que todas as variáveis dependem de $p$, o que não é visto na aproximação de campo médio simples. Em particular, vemos que a densidade $\rho_{c}$ onde ocorre a transição de fase varia com $p$, como observado nos dados de simulação mostrados no gráfico 3.3.

Para obter o ponto crítico em função de $p$, é necessário repetir o procedimento acima, perto da região crítica, para vários valores de $p$. Entretanto, notamos que o método torna-se mais instável à medida que nos aproximamos do ponto crítico. Isso acontece devido a uma acumulação de erros numéricos decorrente de alguns fatores: primeiramente, à medida que nos aproximamos da densidade crítica, $P_{2}$ torna-se muito pequena, e essa variável aparece em vários denominadores das equações (3.3), (3.36) ... (3.39); além disso, a cada passo de integração do sistema de equações, calculamos as variáveis $P_{0}, P_{1}, P_{02}, \ldots$ usando as relações de normalização (3.21), (3.22), (3.31) . . em vez de integrar as equações diferenciais correspondentes (as quais podem ser escritas diretamente a partir da tabela 3.1, ou podem ser obtidas derivando (3.21), ...(3.35) e substituindo (3.3), (3.36) ...(3.39)).

Perto do ponto crítico, os erros numéricos associados às condições de normalização são importantes e, extremamente perto do ponto crítico, variáveis que não são calculadas diretamente em cada passo de integração podem assumir valores externos ao intervalo em que elas estão definidas (tipicamente assumindo valores negativos) para valores de $p$ e $\rho$ tais que o sistema ainda deveria estar no estado ativo. Usando um exemplo ilustrativo: a variável $P_{22}$ é calculada, a cada passo, como a diferença entre $P_{2}$ e $\left(P_{20}+P_{21}\right)$. Perto do ponto crítico, essa diferença aproxima-se muito de 0 (conforme a densidade de sítios ativos diminui, a probabilidade de que um sítio ativo esteja cercado por sítios inativos aumenta) e o erro numérico relativo a 


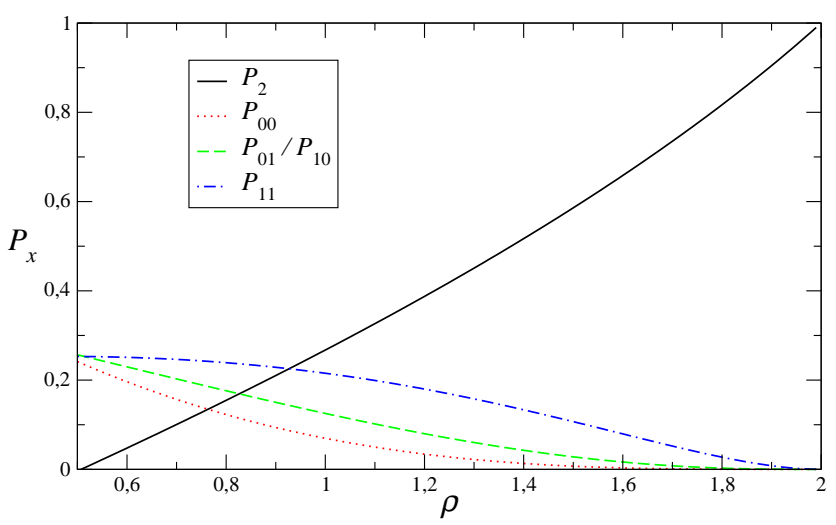

(a) $p=0,01$

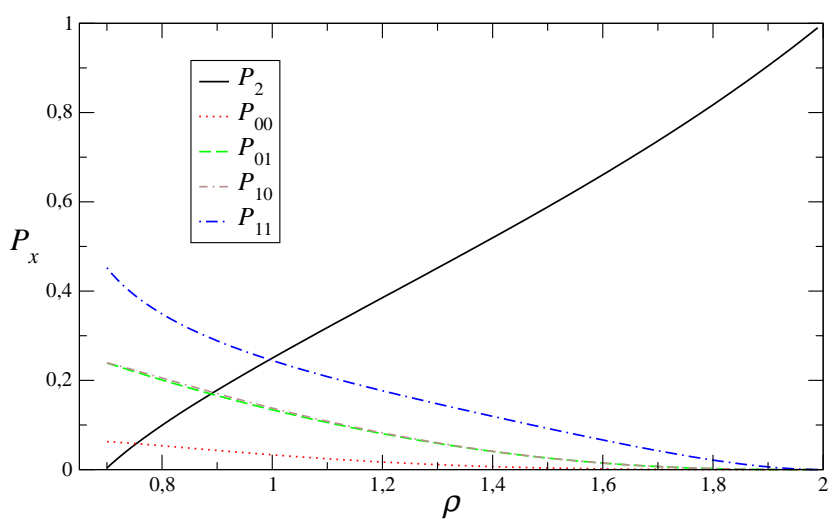

(b) $p=0,3$

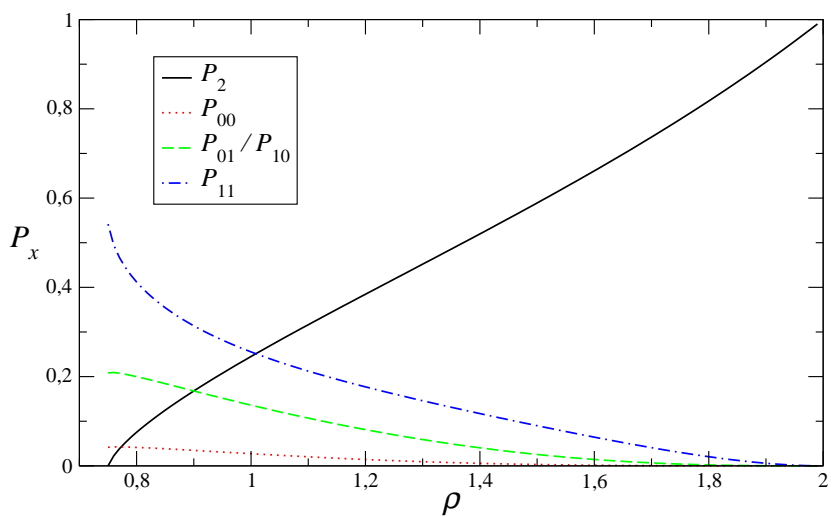

(c) $p=0,5$

Figura 3.4: Valores estacionários de $P_{2}, P_{00}, P_{01}, P_{10}$ e $P_{11}$ obtidos por aproximação de campo médio de pares para alguns valores de $p$, no estado estacionário ativo. 
esse cálculo explode, de modo que $P_{22}$ pode assumir valores externos a seu intervalo de definição $\left(0, P_{2}\right)$.

Uma opção possível para evitar estes problemas é integrar numericamente as equações de evolução temporal de todas as variáveis de 1 e 2 sítios. Entretanto, este método também possui pontos negativos: as condições de normalização deixam de ser satisfeitas exatamente conforme avançamos no tempo, e a densidade de partículas deixa de ser um parâmetro fixo, podendo flutuar sem limites. Outra opção é utilizar métodos mais avançados de integração numérica. Existem vários métodos mais robustos numericamente, dentre os quais podemos destacar o método de Range-Kutta de $4^{\mathrm{a}}$ ordem e métodos preditor-corretor [26], que podem ser utilizados para integrar numericamente conjuntos de equações diferenciais de $1^{\mathrm{a}}$ ordem e obter os valores estacionários de $P_{2}$ e das demais grandezas. Entretanto, em cálculos de campo médio, estamos nomalmente mais interessados em obter descrições qualitativas das propriedades do modelo, de modo que é possível utilizar um método menos robusto, mas que seja mais simples de se implementar.

Para calcular o ponto crítico a partir dos dados contidos nos gráficos $3.4 \mathrm{a} \ldots 3.4 \mathrm{c}$, notamos que $P_{2}$ é uma função linear para $\rho$ pequeno, de modo que o expoente crítico $\beta$ é igual a 1 na aproximação de campo médio de pares. Assim, podemos interpolar uma funçao linear a uma sequência de pontos perto do ponto crítico e calcular sua intersecção com o eixo das abcissas, o que nos fornece uma boa aproximação para $\rho_{c}$, uma vez que essa aproximação é obtida de dados pouco contaminados por erros numéricos. Integrando numericamente as equações (3.3), (3.36), (3.37), (3.38) e (3.39) para outros valores de $p, 0<p \leq 0,5$, perto do ponto crítico, construindo os gráficos correspondentes de $P_{2}^{(p)}$ em função de $\rho$ e extrapolando as funções obtidas por regressão linear, construímos o gráfico da figura 3.5, que mostra a densidade crítica em função de $p$, nas aproximações de campo médio de primeira e segunda ordem.

Os resultados da figura 3.5 também foram obtidos por outro método, que consiste em assumir que as derivadas temporais presentes nas equações (3.3) e (3.36) a (3.39) são nulas e transformar as equações obtidas pela aproximação de campo médio de pares em equações de ponto fixo, da forma:

$$
\begin{aligned}
P_{2} & =f_{1}\left(P_{2}, P_{00}, P_{01}, P_{10}, P_{11}\right), \\
P_{00} & =f_{2}\left(P_{2}, P_{00}, P_{01}, P_{10}, P_{11}\right), \ldots
\end{aligned}
$$




\subsection{O MODELO PAA II - APROXIMAÇÕES DE CAMPO MÉDIO}

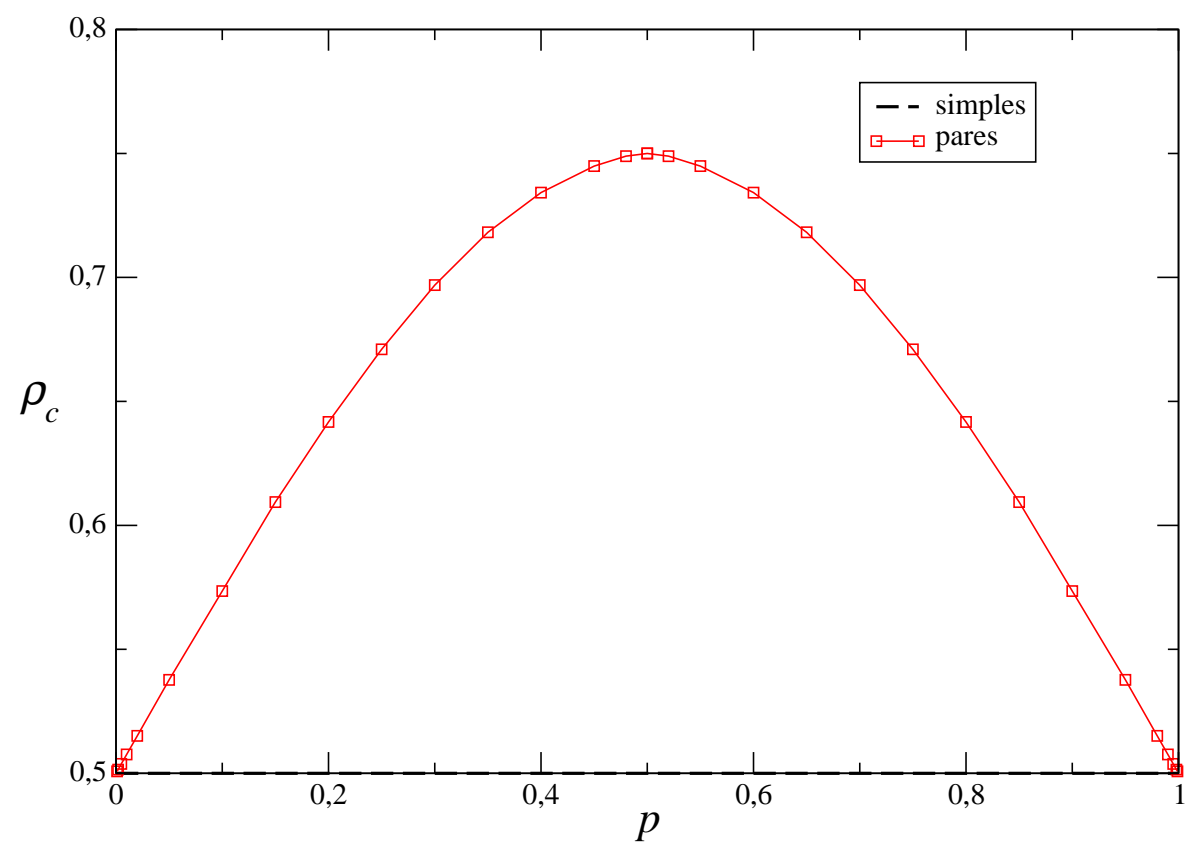

Figura 3.5: Densidade crítica $\rho_{c}$ em função de $p$, na aproximação de campo médio de $1^{\mathrm{a}}$ ordem (linha tracejada) e de $2^{\mathrm{a}}$ ordem (linha contínua).

Equações de ponto fixo podem ser resolvidas de forma iterativa, desde que as funções $f_{n}, 1 \leq n \leq 5$, obedeçam a algumas condições (ver Burden e Faires [26]). Escolhemos valores iniciais das variáveis $P_{2}, P_{00}, \ldots$ e substituímos esses valores nos argumentos das funções $f_{n}$, obtendo novos valores para $P_{2}$ e as demais variáveis, que podem ser substituídos novamente no lado direito das equações de ponto fixo. Se as condições mencionadas em [26] forem satisfeitas, o que pode ser feito rearranjando as funções $f_{n}$, a sequência de aproximações obtida converge para a solução das equações de ponto fixo.

Para cada valor de $p$, iniciamos os cálculos fixando $\rho=1$ e utilizamos como aproximação inicial para $P_{2}$ seu valor estacionário na aproximação de campo médio simples para $\rho=1$, enquanto os valores iniciais das demais variáveis foram calculados usando a aproximação de campo médio simples. Tendo obtido as soluções das equações de ponto fixo para $\rho=1$, diminuímos a densidade de 0,01 e usamos os valores obtidos anteriormente como novas aproximações iniciais. Repetimos o procedimento, diminuindo $\rho$ até $P_{2}$ assumir um valor estacionário negativo. Observamos que, na região próxima ao ponto crítico, o gráfico de $P_{2}$ em função de $\rho$ é aproximadamente linear (ou seja, o expoente $\beta$ é igual a 1 ), de modo que podemos 
usar o método dos mínimos quadrados [26] para ajustar uma reta ao conjunto de pontos. Extrapolando a função obtida para $P_{2}=0$, obtemos $\rho_{c}$ em função de $p$. Os resultados obtidos por este segundo método concordam com os dados na figura 3.5 com uma diferença menor que $2 \%$.

Analisando a figura 3.5, vemos que as duas aproximações de campo médio não apresentam singularidades na densidade crítica quando variamos $p$. No limite $p \rightarrow$ 0 ou 1, as duas aproximações fornecem a mesma densidade crítica $\rho_{c}=0,5$, com $\rho_{c}$ aumentando à medida que nos aproximamos da situação simétrica $p=0,5$. Podemos entender intuitivamente este resultado, uma vez que a densidade de sítios ativos $P_{2}$ diminui durante uma transição quando as duas partículas vão para sítios vazios diferentes (transição simétrica), logo, se comparamos dois sistemas com a mesma densidade e um único sítio ativo, quando a probabilidade da transição simétrica ocorrer é em média menor, a probabilidade do sistema continuar no estado ativo é maior.

\subsubsection{Aproximação de campo médio de trincas}

É possível melhorar a aproximação de campo médio de pares, descrita na seção anterior. Uma vez que as taxas de transição entre configurações no nosso modelo dependem de trincas de partículas, é natural estender a aproximação de modo que as probabilidades de trincas possam ser calculadas de modo independente.

$\mathrm{Na}$ aproximação de campo médio de trincas, tomamos como variáveis independentes as prbabilidades de sítios isolados, de pares e de trincas de sítios vizinhos, sujeitas às condições de normalização e de conservação da densidade de partículas. Além das 5 variáveis $P_{2}, P_{00}, P_{01}, P_{10}$ e $P_{11}$, as novas condições de normalização permitem que escolhemos outras 12 variáveis independentes dentre as 27 densidades de trincas de sítios possíveis. Escolhemos como variáveis independentes: $P_{000}, P_{001}$, $P_{010}, P_{011}, P_{020}, P_{021}, P_{100}, P_{101}, P_{110}, P_{111}, P_{120}$ e $P_{121}$, enquanto as demais variáveis são dadas por:

$$
\begin{aligned}
& P_{002}=P_{00}-P_{000}-P_{001} \\
& P_{012}=P_{01}-P_{010}-P_{011} \\
& P_{022}=P_{02}-P_{020}-P_{021}=1-\rho+P_{2}-P_{00}-P_{01}-P_{020}-P_{021} \\
& P_{102}=P_{10}-P_{100}-P_{101} \\
& P_{112}=P_{11}-P_{110}-P_{111}
\end{aligned}
$$




$$
\begin{aligned}
P_{122} & =P_{12}-P_{120}-P_{121}=\rho-2 P_{2}-P_{10}-P_{11}-P_{120}-P_{121} \\
P_{200} & =P_{00}-P_{000}-P_{100} \\
P_{201} & =P_{01}-P_{001}-P_{101} \\
P_{202} & =P_{02}-P_{002}-P_{102} \\
& =1-\rho+P_{2}-2 P_{00}-P_{01}-P_{10}+P_{000}+P_{001}+P_{100}+P_{101} \\
P_{210} & =P_{10}-P_{010}-P_{110} \\
P_{211} & =P_{11}-P_{011}-P_{111} \\
P_{212} & =P_{12}-P_{012}-P_{112} \\
& =\rho-2 P_{2}-P_{01}-P_{10}-2 P_{11}+P_{010}+P_{011}+P_{110}+P_{111} \\
P_{220} & =P_{20}-P_{020}-P_{120}=1-\rho+P_{2}-P_{00}-P_{10}-P_{020}-P_{120} \\
P_{221} & =P_{21}-P_{021}-P_{121}=\rho-2 P_{2}-P_{01}-P_{11}-P_{021}-P_{121} \\
P_{222} & =P_{22}-P_{022}-P_{122} \\
& =-2+3 P_{2}+2\left(P_{00}+P_{01}+P_{10}+P_{11}\right)+P_{020}+P_{021}+P_{120}+P_{121}
\end{aligned}
$$

Usando a tabela 3.1, escrevemos as equações de evolução temporal para as novas variáveis independentes:

$$
\begin{aligned}
\frac{d}{d t} P_{000} & =p^{2} P_{0200}+(1-p)^{2} P_{0020}-p(2-p) P_{0002}-\left(1-p^{2}\right) P_{2000} \\
\frac{d}{d t} P_{001} & =p^{2} P_{0201}+(1-p)^{2} P_{0021}+2 p(1-p) P_{0002}-p(2-p) P_{0012} \\
& -\left(1-p^{2}\right) P_{2001} \\
\frac{d}{d t} P_{010} & =p^{2} P_{0210}+(1-p)^{2} P_{0120}+2 p(1-p)\left[P_{0020}+P_{0021}+P_{0200}+P_{1200}\right] \\
& -p(2-p) P_{0102}-\left(1-p^{2}\right) P_{2010} \\
\frac{d}{d t} P_{011} & =p^{2} P_{0211}+(1-p)^{2} P_{0121}+2 p(1-p)\left[P_{0022}+P_{0102}+P_{0201}+P_{1201}\right] \\
& -p(2-p) P_{0112}-\left(1-p^{2}\right) P_{2011} \\
\frac{d}{d t} P_{020} & =p^{2} P_{002}+(1-p)^{2} P_{200}+2 p(1-p)\left[P_{0120}+P_{0121}+P_{0210}+P_{1210}\right] \\
& +\left(1-2 p+2 p^{2}\right) P_{0220}-P_{020}-p(2-p) P_{0202}-\left(1-p^{2}\right) P_{2020} \\
\frac{d}{d t} P_{021} & =P_{0221}+p^{2} P_{012}+(1-p)^{2} P_{201}+2 p(1-p)\left[P_{0202}+P_{0122}+P_{0220}+\right. \\
& \left.+P_{0211}+P_{1211}\right]-P_{021}-p(2-p) P_{0212}-\left(1-p^{2}\right) P_{2021}
\end{aligned}
$$




$$
\begin{aligned}
\frac{d}{d t} P_{100} & =p^{2} P_{1200}+(1-p)^{2} P_{1020}+2 p(1-p) P_{2000}-p(2-p) P_{1002} \\
& -\left(1-p^{2}\right) P_{2100} \\
\frac{d}{d t} P_{101} & =p^{2} P_{1201}+(1-p)^{2} P_{1021}+2 p(1-p)\left[P_{020}+P_{1002}+P_{2001}\right] \\
& -p(2-p) P_{1012}-\left(1-p^{2}\right) P_{2101} \\
\frac{d}{d t} P_{110} & =p^{2} P_{1210}+(1-p)^{2} P_{1120}+2 p(1-p)\left[P_{1020}+P_{1021}+P_{2010}+P_{2200}\right] \\
& -p(2-p) P_{1102}-\left(1-p^{2}\right) P_{2110} \\
\frac{d}{d t} P_{111} & =p^{2} P_{1211}+(1-p)^{2} P_{1121}+2 p(1-p)\left[P_{1022}+P_{1102}+P_{2011}+P_{2201}\right] \\
& -p(2-p) P_{1112}-\left(1-p^{2}\right) P_{2111} \\
\frac{d}{d t} P_{120} & =P_{1220}+p^{2} P_{102}+(1-p)^{2} P_{210}+2 p(1-p)\left[P_{0220}+P_{1120}+P_{1121}\right. \\
& \left.+P_{2020}+P_{2210}\right]-P_{120}-p(2-p) P_{1202}-\left(1-p^{2}\right) P_{2120} \\
\frac{d}{d t} P_{121} & =p^{2} P_{112}+(1-p)^{2} P_{211}+2 p(1-p)\left[P_{0221}+P_{1122}+P_{1202}+P_{1220}\right. \\
& \left.+P_{2021}+P_{2211}\right]+\left(1+2 p-2 p^{2}\right) P_{1221}-P_{121}-p(2-p) P_{1212} \\
& -\left(1-p^{2}\right) P_{2121}
\end{aligned}
$$

Vemos que, nas equações de evolução de trincas, aparecem termos correspondentes a quartetos de sítios. Tomamos a aproximação

$$
P_{x y z w}=\frac{P_{x y z} P_{y z w}}{P_{y z}}
$$

que exprime cada densidade de quarteto em função de variáveis já conhecidas. Tal aproximação pode ser justificada com um raciocínio análogo ao utilizado para justificar a aproximação de campo médio de pares.

Usando essa aproximação, reescrevemos as equações (3.58) a (3.69) em termos do conjunto de variáveis $P_{2}, P_{00} \ldots P_{11}, P_{000} \ldots P_{121}$. É possível escolher outros conjuntos de variáveis, entretanto, o conjunto acima permite que escrevemos as equações de evolução temporal de forma mais simples. Integrando numericamente as equações (3.3), (3.36) a (3.39) e (3.58) a (3.69) pelo método de Euler até que não seja mais possível observar uma variação com o tempo do conjunto de variáveis (ou seja, o sistema alcançou o estado estacionário), obtemos $P_{2}$ em função de $\rho$ e $p$. Utilizando análise de regressão linear perto do ponto crítico e extrapolando a 
função obtida até cruzar com o eixo $x$, obtemos $\rho_{c}$, para cada $p$, como feito no item anterior. O gráfico 3.6 mostra um exemplo de cálculo, para $p=0,35$.

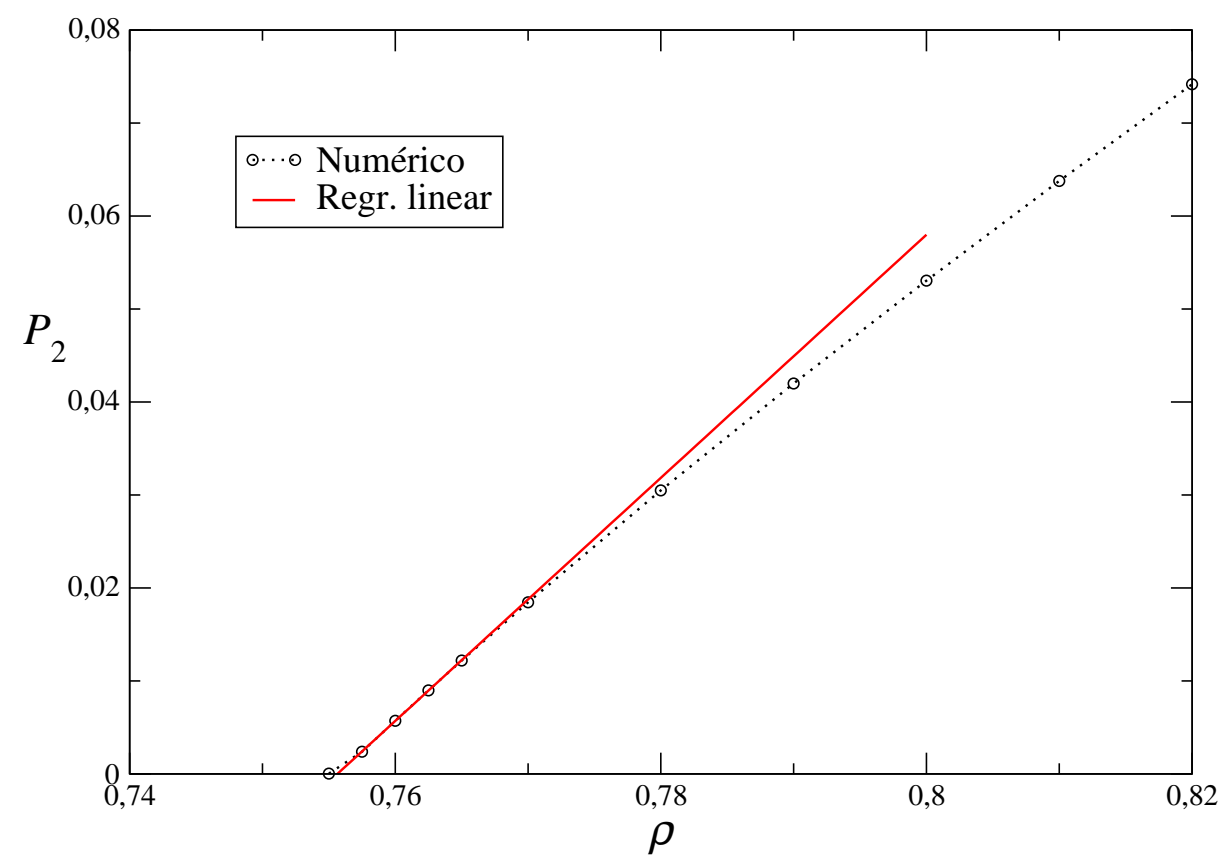

Figura 3.6: Densidade de sítios ativos $P_{2}$ calculada por aproximação de campo médio de trincas para $p=0,35$, pelo primeiro conjunto de equações (círculos ligados por linha pontilhada) e função interpoladora obtida por regressão linear na região entre $\rho=0,758$ e $\rho=0,764$ (linha contínua). A densidade crítica obtida por este método é igual a 0,7557 .

Observamos que, nesta ordem de aproximação, o método de Euler é menos preciso. Aplicando esse método para calcular $\rho_{c}$, somos forçados a tomar maiores cuidados na análise dos dados obtidos, devido às complicações envolvendo o cálculo de probabilidades de trincas pela condições de normalização (3.43) a (3.57) e ao maior número de equações.

Para verificar se os resultados obtidos são consistentes, podemos utilizar outros métodos de integração. Primeiramente, tentamos resolver numericamente o sistema não-linear de equações obtido quando se igualam todas as derivadas temporais a zero, transformando cada equação numa equação de ponto fixo e implementando o esquema de aproximações sucessivas descrito no item 3.4.2, sem sucesso. Métodos numéricos para resolver sistemas de equações necessitam de aproximações iniciais suficientemente precisas para que a solução numérica convirja para o conjunto de valores procurado. Além disso, o sistema de equações deve ser reescrito numa forma tal 
que sucessivas aproximações convirjam para o resultado desejado. Devido a dificuldades externas ao problema (falta de tempo para aprender a utilizar outros métodos e para implementar os programas), optamos por tentar resolver este problema sem abandonar o método de Euler. Optamos por escolher novas variáveis e reescrever o conjunto de equações conforme esta escolha. Como estamos interessados no comportamento do sistema perto do ponto crítico, o valor estacionário da probabilidade de qualquer sequência (pares ou trincas) composta por ao menos um sítio ativo deve ser pequeno quando comparado ao de uma sequência com o mesmo número de sítios mas sem sítios ativos. Escolhendo estas probabilidades como variáveis do problema, não corremos o risco de um pequeno desvio nas variáveis integradas traduzir-se num erro catastrófico nas demais variáveis, calculadas por normalização e esperamos que os resultados obtidos, mesmo utilizando o método de Euler (que é inferior em termos de robustez a outros métodos mais recentes [26]) sejam consistentes.

Escolhemos, para a segunda etapa, as variáveis $P_{2}, P_{22}, P_{21}, P_{12}, P_{11}, P_{222}, P_{221}$, $P_{212}, P_{211}, P_{202}, P_{201}, P_{122}, P_{121}, P_{112}, P_{111}, P_{102}$ e $P_{101}$. As equações de evolução desse conjunto de variáveis são:

$$
\begin{aligned}
\frac{d}{d t} P_{12} & =P_{122}+p^{2} P_{102}+(1-p)^{2} P_{21}+2 p(1-p)\left(P_{022}+P_{202}+P_{221}\right) \\
& +p(2-p) P_{112}-P_{12}-(1-p)^{2} P_{212} \\
\frac{d}{d t} P_{21} & =P_{221}+p^{2} P_{12}+(1-p)^{2} P_{201}+2 p(1-p)\left(P_{122}+P_{202}+P_{220}\right)+ \\
& +\left(1-p^{2}\right) P_{211}-P_{21}-p(2-p) P_{212} \\
\frac{d}{d t} P_{22}= & \left(1-2 p+2 p^{2}\right) P_{202}+\left(1+2 p-2 p^{2}\right)\left(P_{212}+P_{222}-P_{22}\right) \\
\frac{d}{d t} P_{102}= & p^{2}\left(P_{1002}+P_{1202}\right)+(1-p)^{2}\left(P_{120}+P_{1022}\right)+2 p(1-p)\left(P_{021}+\right. \\
& \left.+P_{2002}\right)+p(2-p) P_{1012}-P_{102}-\left(1-p^{2}\right) P_{2102} \\
\frac{d}{d t} P_{112}= & p^{2}\left(P_{1102}+P_{1212}\right)+(1-p)^{2}\left(P_{121}+P_{1122}\right)+2 p(1-p)\left(P_{022}+\right. \\
& \left.+P_{2012}+P_{2202}\right)+p(2-p) P_{1112}-P_{112}-\left(1-p^{2}\right) P_{2112} \\
\frac{d}{d t} P_{122}= & p^{2} P_{1202}+(1-p)^{2} P_{212}+2 p(1-p)\left(P_{0222}+P_{2022}+P_{2212}\right)+ \\
+ & p(2-p) P_{1212}+\left(1+2 p-2 p^{2}\right) P_{1222}-\left(1-p^{2}\right) P_{2122}+ \\
- & \left(1+2 p-2 p^{2}\right) P_{122}
\end{aligned}
$$




$$
\begin{aligned}
\frac{d}{d t} P_{201}= & p^{2}\left(P_{021}+P_{2201}\right)+(1-p)^{2}\left(P_{2001}+P_{2021}\right)+2 p(1-p)\left(P_{120}+\right. \\
& \left.+P_{2002}\right)+\left(1-p^{2}\right) P_{2101}-P_{201}-p(2-p) P_{2012} \\
\frac{d}{d t} P_{202}= & p^{2}\left(P_{022}+P_{2202}\right)+(1-p)^{2}\left(P_{220}+P_{2022}\right)+2 p(1-p) P_{121}+ \\
& +p(2-p) P_{2012}+\left(1-p^{2}\right) P_{2102}+\left(1-2 p+2 p^{2}\right) P_{2002}-2 P_{202} \\
\frac{d}{d t} P_{211}= & p^{2}\left(P_{121}+P_{2211}\right)+(1-p)^{2}\left(P_{2011}+P_{2121}\right)+2 p(1-p)\left(P_{220}+\right. \\
& \left.+P_{2022}+P_{2102}\right)+\left(1-p^{2}\right) P_{2111}-P_{211}-p(2-p) P_{2112} \\
\frac{d}{d t} P_{212}= & p^{2}\left(P_{2102}+P_{2212}\right)+(1-p)^{2}\left(P_{2012}+P_{2122}\right)+p(2-p) P_{122}+ \\
+ & \left(1-p^{2}\right) P_{221}+\left(1+2 p-2 p^{2}\right) P_{2112}-2 P_{212} \\
\frac{d}{d t} P_{221}= & p^{2} P_{212}+(1-p)^{2} P_{2021}+2 p(1-p)\left(P_{2122}+P_{2220}+P_{2202}\right)+ \\
+ & \left(1-p^{2}\right) P_{2121}+\left(1+2 p-2 p^{2}\right) P_{2221}-p(2-p) P_{2212}+ \\
- & \left(1+2 p-2 p^{2}\right) P_{221} \\
\frac{d}{d t} P_{222}= & p^{2} P_{2202}+(1-p)^{2} P_{2022}+p(2-p) P_{2212}+\left(1-p^{2}\right) P_{2122}+ \\
+ & \left(1+2 p-2 p^{2}\right)\left(P_{2222}-P_{222}\right)
\end{aligned}
$$

em conjunto com as equações (3.3), (3.39), (3.65), (3.67) e (3.69), que descrevem a evolução temporal de $P_{2}, P_{11}, P_{101}, P_{111}$ e $P_{121}$.

Como anteriormente, escolhemos como condições iniciais uma distribuição aleatória de partículas com a densidade $\rho$ fixada. Integramos o conjunto de equações diferenciais numericamente, utilizando $\Delta t=0,001$, até alcançarmos o estado estacionário, e obtivemos gráficos $P_{2} \times \rho, P_{21} \times \rho$ e $P_{22} \times \rho$, para vários valores de $p$. O intervalo de tempo necessário para que o sistema alcance o estado estacionário, para a maioria dos valores de $p$ e $\rho$, variou entre 100 e 200 unidades de tempo.

Ao analisar os dados obtidos, observamos que, para $p \rightarrow 0$, o método de integração fornece resultados menos confiáveis. Observou-se a estabilização do conjunto de variáveis apenas numa faixa de valores de $\rho$ muio próxima do ponto crítico. Para $p \gtrsim 0,15$, o problema deixa de ser relevante porque, embora este conjunto de equações continue fornecendo resultados impossíveis para $\rho$ grande, conseguimos obter dados para toda a região perto do ponto crítico na qual $P_{2}$ é linear.

Tendo obtido os gráficos $P_{2} \times \rho, P_{21} \times \rho$ e $P_{22} \times \rho$, a densidade crítica pode ser obtida de três formas: interpolando uma função linear ao gráfico $P_{2} \times \rho$, e 
extrapolando essa função até cruzar a origem, aplicando o mesmo método ao gráfico $P_{21} \times \rho$, ou interpolando uma função quadrática ao gráfico $P_{22} \times \rho$ :

$$
P_{22}=q_{2} \rho^{2}+q_{1} \rho+q_{0}
$$

na mesma região de densidades e calculando a raiz de módulo maior. Isso ocorre porque, perto da densidade crítica, $P_{2}=\epsilon$ é muito menor que $P_{1}$ ou $P_{0}$, e podemos expandir as demais grandezas em função de $\epsilon$, obtendo:

$$
\begin{aligned}
& P_{21}=a_{1} \epsilon+\sum_{n=2}^{\infty} a_{n} \epsilon^{n} \\
& P_{22}=b_{2} \epsilon^{2}+\sum_{n=3}^{\infty} b_{n} \epsilon^{n}
\end{aligned}
$$

Os gráficos 3.7a e 3.7b mostram como foi calculada a densidade crítica por cada método para $p=0,35$. Notamos que o cálculo da densidade crítica a partir do gráfico $P_{22} \times \rho$ possui uma precisão menor que o feito a partir dos demais gráficos, uma vez que, para que a expansão (3.85) esteja correta, a primeira derivada desse gráfico deve se anular no ponto crítico: $P_{22}(\rho)=P_{22}^{\prime}(\rho)=0$. Na prática, o discrimante da função interpolante $\Delta=q_{1}^{2}-4 q_{2} q_{0}$ é ligeiramente maior do que zero e a função possui duas raízes diferentes, em vez de uma única raiz. nessa situação, escolhemos a maior raiz como a densidade crítica, como se vê no gráfico 3.7b. Os gráficos 3.7a e 3.7b mostram como foi calculada a densidade crítica por cada método para $p=0,35$.

Comparando as figuras 3.6, 3.7a e 3.7b, vemos que o valor de $\rho_{c}$ obtido por todos os métodos é consistente. Analisando os gráficos obtidos para diversos valores de $p$, constatamos que a diferença entre os valores de $\rho_{c}$ obtidos por cada método, para cada valor de $p$, é da ordem de 0,001. Tomando uma média dos valores obtidos pelos diferentes métodos, construímos a figura 3.8, com o gráfico de $\rho_{c}$ como função de $p$.

Analisando a figura 3.8, vemos que, qualitativamente, as aproximações de campo médio de pares e de trincas são muito similares: as duas curvas são analíticas para todos os valores de $p$, possuindo um máximo no ponto $p=0,5$. No limite $p \rightarrow 0$ ou 1 , os valores obtidos são muito próximos, e a diferença aumenta conforme nos aproximamos do eixo de simetria dos gráficos $p=0,5$.

Também vemos que escrever e resolver numericamente um conjunto de equações diferenciais descrevendo aproximações de campo médio de ordens superiores envolve 


\subsection{O MODELO PAA II - APROXIMAÇÕES DE CAMPO MÉDIO}

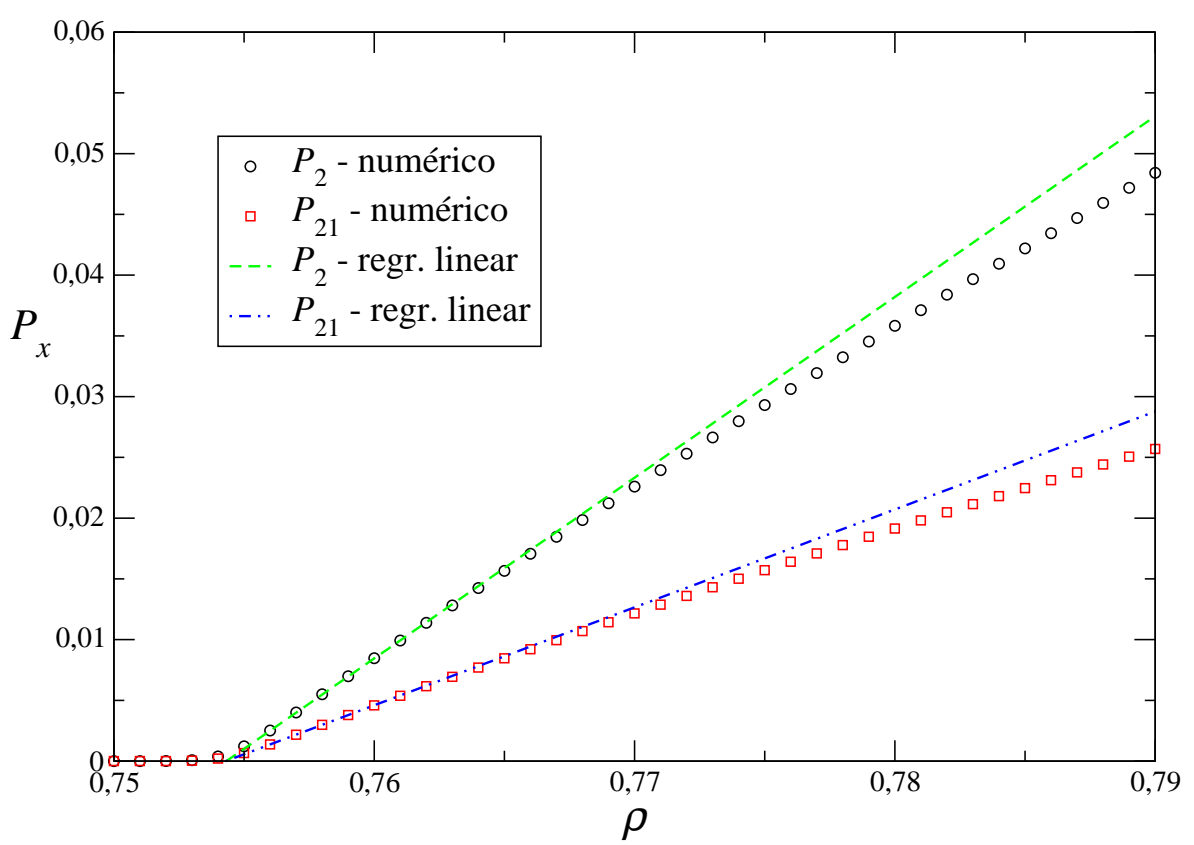

(a) $P_{2}$ e $P_{21}$

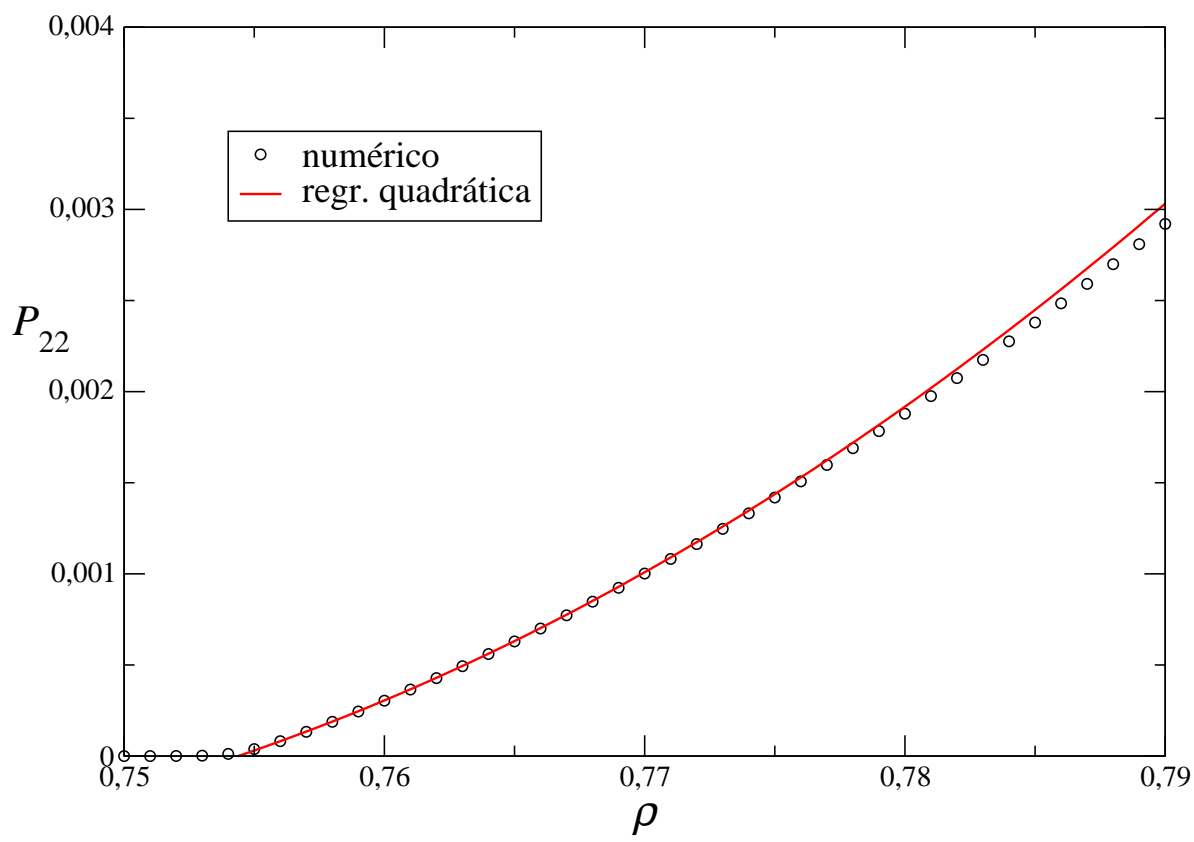

(b) $P_{22}$

Figura 3.7: Determinação da densidade crítica por aproximação de campo médio de trincas, utilizando o segundo conjunto de equações, para $p=0,35$, por (a) ajuste de reta aos gráficos de $P_{2}$ e $P_{21}$ entre $\rho=0,756$ e $\rho=0,76$ e (b) ajuste de função quadrática ao gráfico de $P_{22}$ entre $\rho=0,758$ e $\rho=0,765$. Obtemos, pelo método (a), $\rho_{c}=0,7543$ e, pelo método (b), $\rho_{c}=0,7544$. 


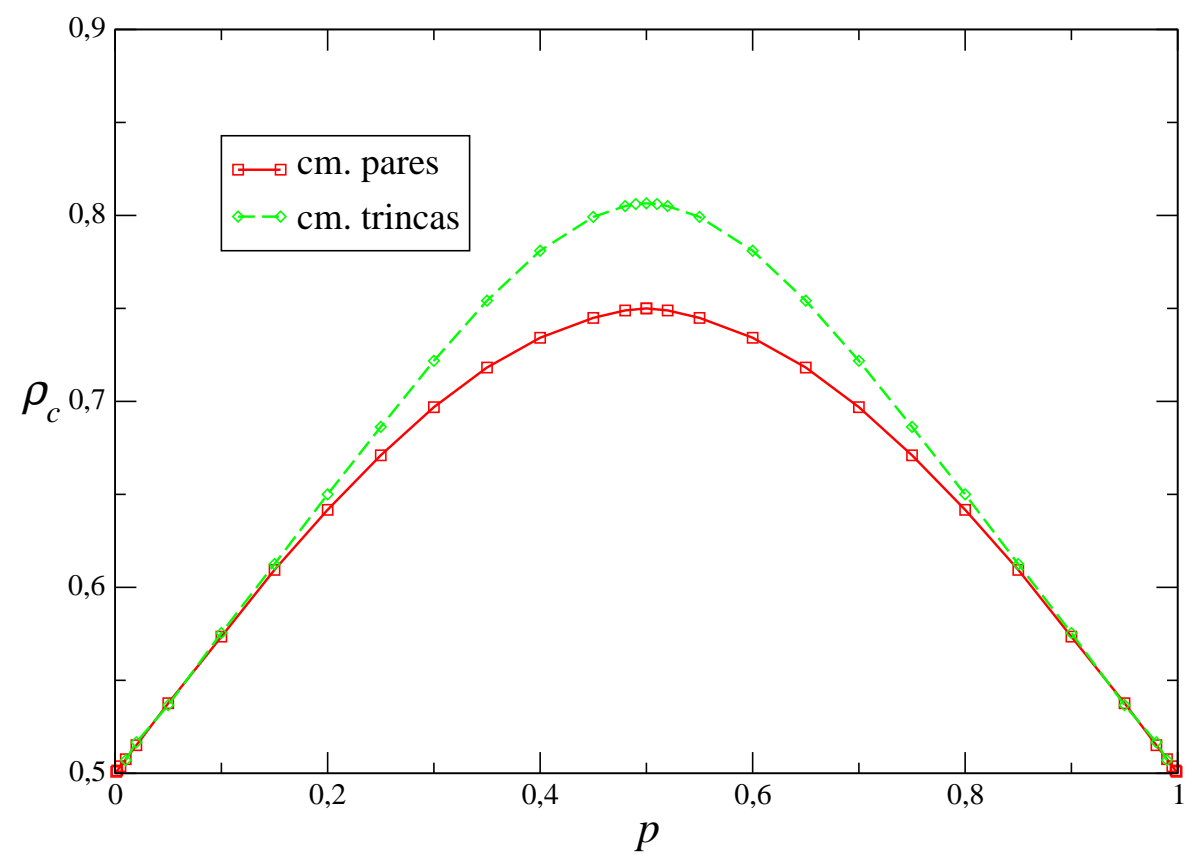

Figura 3.8: Densidade crítica $\rho_{c}$ em função de $p$, na aproximação de campo médio de pares e de trincas. Os símbolos (quadrado, losango) marcam valores obtidos por integração numérica e linearização, enquanto as linhas representam o comportamento geral dos gráficos.

um custo (de tempo e poder computacional) que aumenta muito rapidamente à medida que a ordem da aproximação aumenta, de modo que, para o modelo que estamos estudando, não compensa escrever aproximações de campo médio de ordem superior à de trincas.

\subsubsection{Aproximações para o fluxo de partículas}

Podemos utilizar a aproximação de campo médio simples para calcular o fluxo de partículas no estado estacionário ativo. Utilizando a aproximação (3.19) para simplificar (3.18), obtemos:

$$
\phi=(1-2 p) P_{2}\left(1-P_{2}\right)+\left[\left(1-2 p+p^{2}\right)-p^{2}\right] P_{2} P_{0}=(1-2 p)(2-\rho) P_{2}
$$

Tomando

$$
P_{2}= \begin{cases}0, & 0 \leq \rho<0,5 \\ 2-\sqrt{5-2 \rho}, & 0,5 \leq \rho \leq 2\end{cases}
$$




\subsection{O MODELO PAA II - APROXIMAÇÕES DE CAMPO MÉDIO}

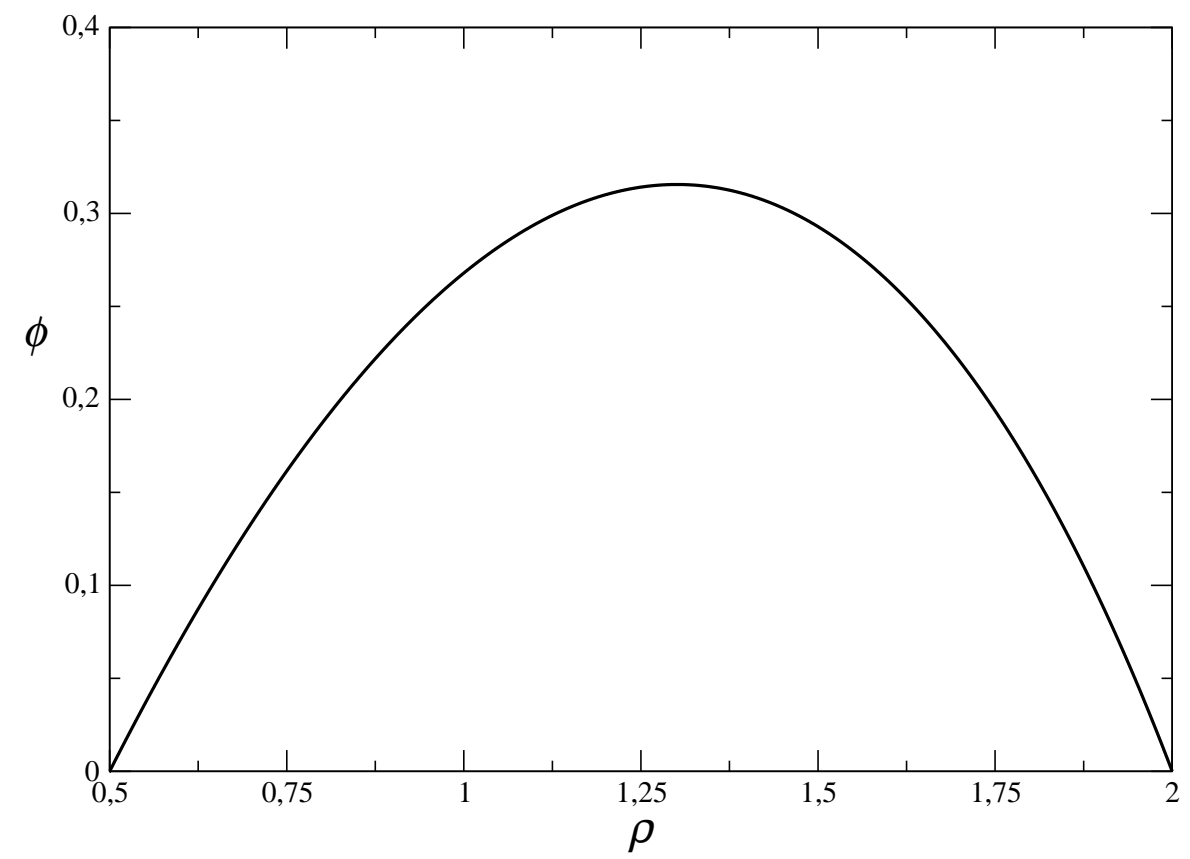

Figura 3.9: Variação do fluxo calculado pela aproximação de campo médio simples em função de $\rho$, para $p=0$. O fluxo é nulo para $0 \leq \rho \leq 0,5$.

obtemos a variação do fluxo com $\rho$ e $p$. A figura 3.9 mostra a variação de $\phi \operatorname{com} \rho$, assumindo que $p=0$.

O sinal do resultado (3.86) é invertido pela troca $p \rightarrow 1-p$. Entretanto, como essa é a única dependência do fluxo com o parâmetro $p$, a aproximação de campo médio simples falha em reproduzir vários aspectos do modelo, como a variação da densidade crítica (aqui entendida como a densidade em que o fluxo cai a zero) com $p$. Em vez disso, observa-se que o fluxo se anula em $\rho=0,5$, para qualquer valor de $p$.

Na fase ativa, o fluxo apresenta um máximo na variável $\rho$, calculado como:

$$
\frac{d}{d \rho} \phi^{(1)}=0 \rightarrow 9 \rho^{2}-34 \rho+29=0
$$

A raiz fisicamente aceitável desta equação é $\rho=(17-2 \sqrt{7}) / 9 \cong 1,301$. Neste ponto, a densidade de sítios ativos (que também não depende de $p$ nesta aproximação) é:

$$
P_{2}=2-\frac{\sqrt{11+4 \sqrt{7}}}{3} \cong 0,4514
$$


e o fluxo é dado por:

$$
\phi_{\max }^{(1)}=(1-2 p) \frac{(1+2 \sqrt{7})(6-\sqrt{11+4 \sqrt{7}})}{27} \approx 0,3156(1-2 p)
$$

Como o valor máximo de $(1-2 p)$ é igual a 1 , o fluxo máximo é aproximadamente igual a 0,3156 , ocorrendo para $\rho \approx 1,301$. Para valores menores de $\rho$, o fluxo cai devido à pequena densidade de sítios ativos (que podem expelir uma partícula). Para valores maiores de $\rho$, ele também diminui, uma vez que ocorre um excesso de sítios ativos e uma falta de sítios de destino de partículas.

Para observar uma dependência mais precisa de $\phi \operatorname{com} p$ e $\rho$, é necessário usar aproximações superiores à de campo médio simples. Tendo obtido, nas seções 3.4.2 e 3.4.3, estimativas numéricas para $P_{2}$ e as densidades de pares, podemos substituir essas estimativas em (3.17) (ou (3.18)) para calcular o fluxo de partículas para diversos valores de $p$ no intervalo $0<p<0,5$ e para diversos valores de $\rho$ no intervalo $\rho_{c}(p)<\rho<2$, utilizando as aproximações de campo médio de pares e de trincas. Os gráficos obtidos para alguns valores de $p$, utilizando as aproximações de pares e de trincas são mostrados, juntamente com o gráfico obtido anteriormente para a aproximação de campo médio simples, para fins de comparação, nas figuras 3.10a, 3.10b e 3.10c.

Vemos que, nos limites $\rho \rightarrow \rho_{c}, \rho \rightarrow 2$ ou $p \rightarrow 0,5$, o fluxo de partículas tende a zero, como é de se esperar intuitivamente: quando $\rho=\rho_{c}$, o número de partículas ativas se anula no estado estacionário e não ocorre movimento; quando $\rho \rightarrow 2$, as partículas não realizam movimentos porque os sítios de destino também são ativos e não podem receber mais partículas; e quando $p \rightarrow 0,5$, o fluxo se anula porque o mesmo número de partículas se desloca para a direita e para a esquerda em um período fixo de tempo.

Mantendo $p$ constante, o fluxo máximo ocorre quando $\rho \sim 1,3$, e sua posição varia muito pouco com $p$. Comparando os gráficos 3.3 e 3.10, vemos que, para $\rho \sim 1,3, P_{2} \sim 0,5$, de modo que existe quase o mesmo número de sítios ativos e inativos. Mantendo $\rho$ constante, o fluxo máximo ocorre quando $p=0$, um resultado esperado, pois neste caso as partículas vão apenas para a direita. Nos limites $p \rightarrow 0$, $\rho_{a} \rightarrow 0$ ou $\rho \rightarrow 2$, começam a ocorrer erros de arredondamento que impedem o cálculo do fluxo quando $p \leq 0,01, \rho_{a} \leq 0,001,2-\rho \leq 0,01$.

Comparando as ordens de aproximação utilizadas, vemos que, para $p$ pequeno, os resultados obtidos são similares, exceto na região de alta densidade, onde a apro- 


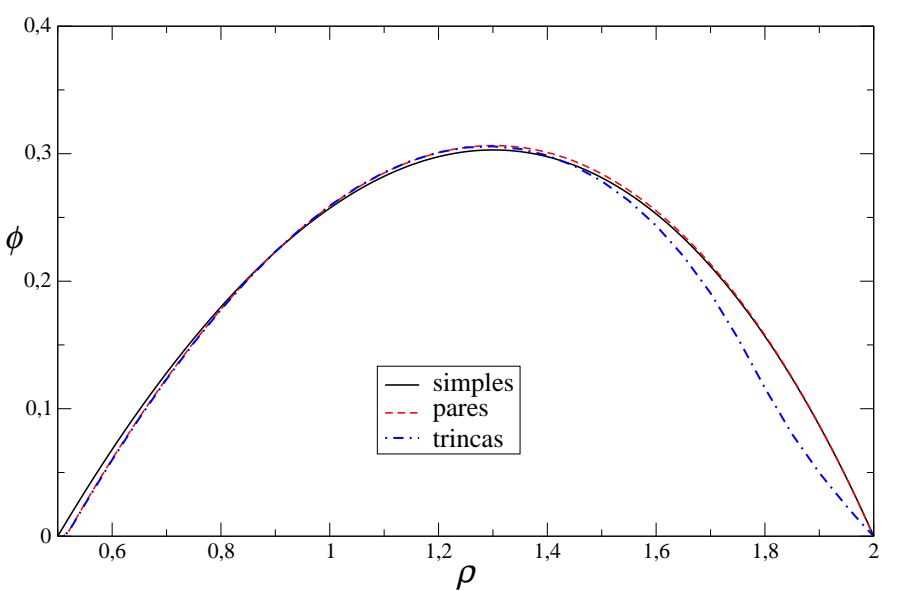

(a) $p=0,02$

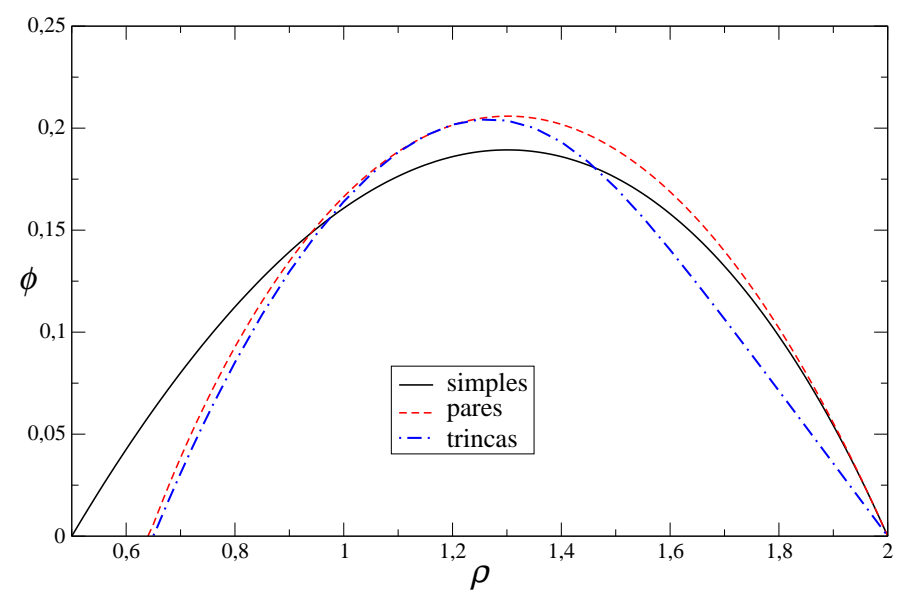

(b) $p=0,2$

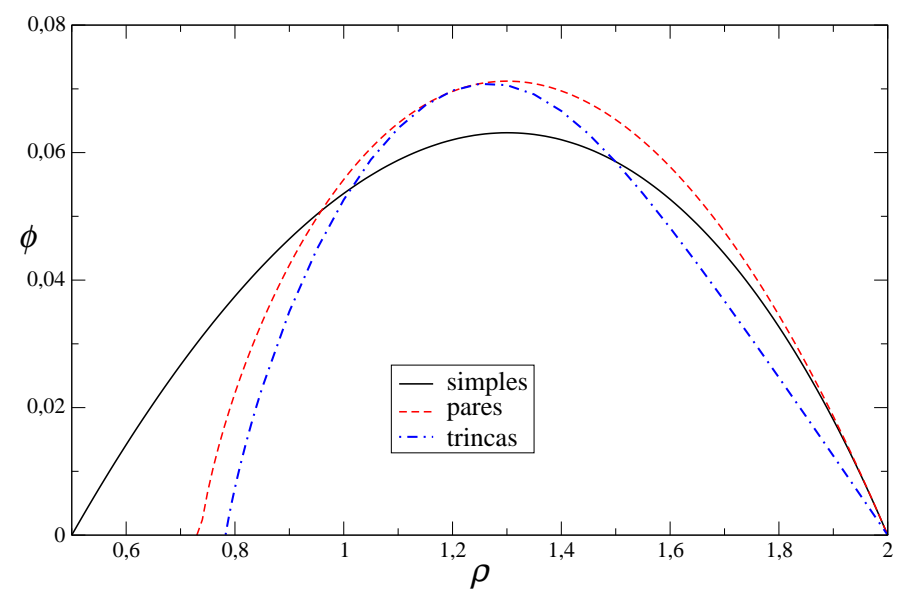

(c) $p=0,4$

Figura 3.10: Fluxo de partículas calculado por aproximações de campo médio simples, de pares e de trincas, para alguns valores de $p$. 
ximação de trincas fornece um fluxo médio menor que as outras. Esta característica se mantém conforme $p$ aumenta. Quando $p$ aumenta, as aproximações superiores de campo médio afastam-se da aproximação mais simples, tanto na descrição da densidade crítica (como visto no capítulo 3.4), como no valor do fluxo máximo. Entretanto, a densidade correspondente ao fluxo máximo mantém-se, nas três ordens, aproximadamente igual a 1,3 .

\subsection{O modelo de pilha de areia assimétrico III - Simulação}

Nesta seção, vamos estudar o modelo de pilha de areia assimétrico por meio de simulação numérica. Vamos estudar a variação de sua densidade crítica em função de $p$, medir expoentes críticos associados à transição e estudar numericamente o fluxo de partículas em função de $p$ e $\rho$.

A simulação numérica de modelos estocásticos é um método poderoso utilizado nas últimas décadas como alternativa a métodos analíticos e combinatórios. O objetivo de uma simulação de um modelo estocástico é construir uma amostra do espaço de fase correspondente ao estado estacionário ativo do sistema e calcular grandezas que descrevem o comportamento do sistema. Para isso, escreve-se um programa que constrói uma configuração inicial para o sistema e aplica as regras de atualização $M$ vezes, onde $M=\left(\Delta t_{0}+\Delta t_{1}\right) M_{0} . \quad M_{0}$ é uma constante do programa igual a uma unidade de tempo: em nosso caso, vamos tomar $M_{0}$ igual a $L$ tentativas de atualização ou um passo de Monte Carlo. $\Delta t_{0}$ é um intervalo de tempo necessário para que todas a densidade de sítios ativos alcance o valor estacionário, independentemente da condição inicial, enquanto $\Delta t_{1}$ é um intervalo de tempo suficiente para que possamos calcular $P_{2}$ e o segundo momento da distribuição estacionária de probabilidade (ver seção 3.5.1) e os resultados obtidos possuam estabilidade numérica. Para percorrer de forma mais abrangente o espaço de fase, nós repetimos a rotina acima $M_{c i}$ vezes, partindo de condições iniciais diferentes.

Uma vez que não é possível simular sistemas de tamanho infinito numericamente, é necessário entender como um sistema de tamanho finito se comporta durante uma simulação numérica. Seja $L$ uma medida do tamanho do sistema: em nosso problema, $L$ é o número de sítios na rede. Todas as grandezas definidas como densidades não são contínuas, mas assumem valores separados entre si por $1 / L$. Assim, a cada 
passo de atualização, tais variáveis são atualizadas, variando de múltiplos de $1 / L$, em vez de variar continuamente. Devido ao tamanho finito da rede, no estado estacionário, as variáveis que descrevem o estado do sistema não são constantes, mas flutuam em torno de um valor médio. Em particular, se simulamos o modelo perto do ponto crítico, de modo que existam apenas alguns sítios ativos no estado estacionário, uma flutuação no valor de $P_{2}$ pode conduzir o sistema para o estado absorvente, introduzindo uma distorção nos cálculo dos valores médios.

Em nosso problema, a densidade de sítios ativos pode se anular para qualquer valor de $\rho$ no intervalo $0 \leq \rho \leq 1$, uma vez que existem configurações nesse intervalo de $\rho$ que não possuem nenhum sítio ativo. Se o número de sítios ativos presentes numa realização do sistema é muito maior que zero, é extremamente improvável observar uma transição para a fase absorvente, de modo que, conforme $L$ aumenta, o intervalo de densidades onde este efeito é importante diminui. Entretanto, perto desse limite, é preciso tomar cuidados específicos para evitar a queda no estado absorvente.

\subsubsection{Densidade crítica}

Para a determinação da densidade crítica, o método direto - calcular $P_{2}$ para valores de $\rho$ ao redor do ponto crítico e verificar para qual $\rho$ essa grandeza se anula - não fornece bons resultados, devido aos problemas citados acima. Assim, vamos utilizar métodos indiretos, baseados na teoria de escala finita (finite-size scaling theory). Para mais detalhes, consultar as referências Newman e Barkena [15] e Cardy [17].

A teoria de escala finita procura estabelecer o comportamento de grandezas termodinâmicas medidas em sistemas de tamanho finito, tipicamente caracterizados por uma dimensão linear $L$, e relacionar esse comportamento ao de um sistema no limite termodinâmico $(L \rightarrow \infty)$. Uma das hipóteses da teoria de escala finita aplicada a sistemas que exibem uma transição de fase para uma fase absorvente estabelece que o parâmetro de ordem ( $P_{2}$ para o nosso modelo) de um sistema finito perto da criticalidade obedeça à relação a seguir:

$$
P_{2}(\rho, L)=L^{-\frac{\beta}{\nu_{\perp}}} \mathcal{F}\left(\epsilon L^{1 / \nu_{\perp}}\right)
$$

onde $\epsilon=\rho-\rho_{c}, \beta$ é o expoente crítico relacionado ao decaimento do parâmetro de ordem perto do ponto crítico para o sistema infinito, $\nu_{\perp}$ é o expoente crítico 
relacionado ao decaimento algébrico da função de correlação espacial no ponto crítico e $\mathcal{F}$ é uma função de escala.

Para que os métodos de análise que vamos utilizar dêem resultados consistentes, é necessário que o sistema permaneça no estado ativo, mesmo abaixo do ponto crítico. Os métodos comumente utilizados para alcançar esta meta reduzem-se a:

- Considerar apenas realizações do sistema tais que ele ainda não caiu no estado absorvente no fim da simulação.

- Modificar as regras do modelo, de modo que o sistema não possa cair no estado absorvente, ou que seja possível conduzir o sistema para fora do estado absorvente sempre que ocorrer a queda. Um exemplo de uso desse critério para a versão simética do modelo PAA de pilha de areia é feito por Tomé e de Oliveira $[28]^{1}$.

O primeiro método implica em desprezar iterações do programa de simulação nas quais ele cai no estado absorvente antes de haver passado um intervalo de tempo mínimo, desperdiçando recursos computacionais. Assim, escolhemos o segundo método, que possui a vantagem de que a mudança nas regras deve afetar apenas uma pequena região em torno do ponto crítico, a qual deve diminuir com o aumento do tamanho $L$ do modelo simulado.

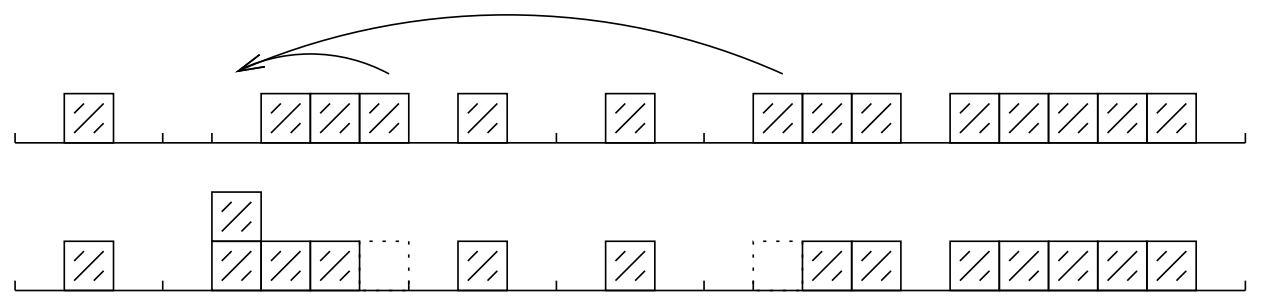

Figura 3.11: Exemplo de alteração de uma configuração que caiu no estado absorvente.

A mudança nas regras do nosso modelo é simples: após cada passo de atualização, verificamos se o número de sítios ativos caiu a zero. Caso isso tenha ocorrido, perturbamos o estado absorvente, escolhendo um sítio ao acaso e transportando uma ou duas partículas de origens aleatórias de modo que o sítio passe a ter 2 partículas, como mostra a figura 3.11. Conforme o tamanho da rede aumenta, as

\footnotetext{
${ }^{1}$ Existem outros métodos análogos a esse para evitar a queda no estado absorvente. Por exemplo, Dickman [18] salva, a intervalos de tempo regulares, a configuração atual. Quando o sistema está prestes a cair no estado absorvente, a configuração é substituída por uma das configurações salvas
} 


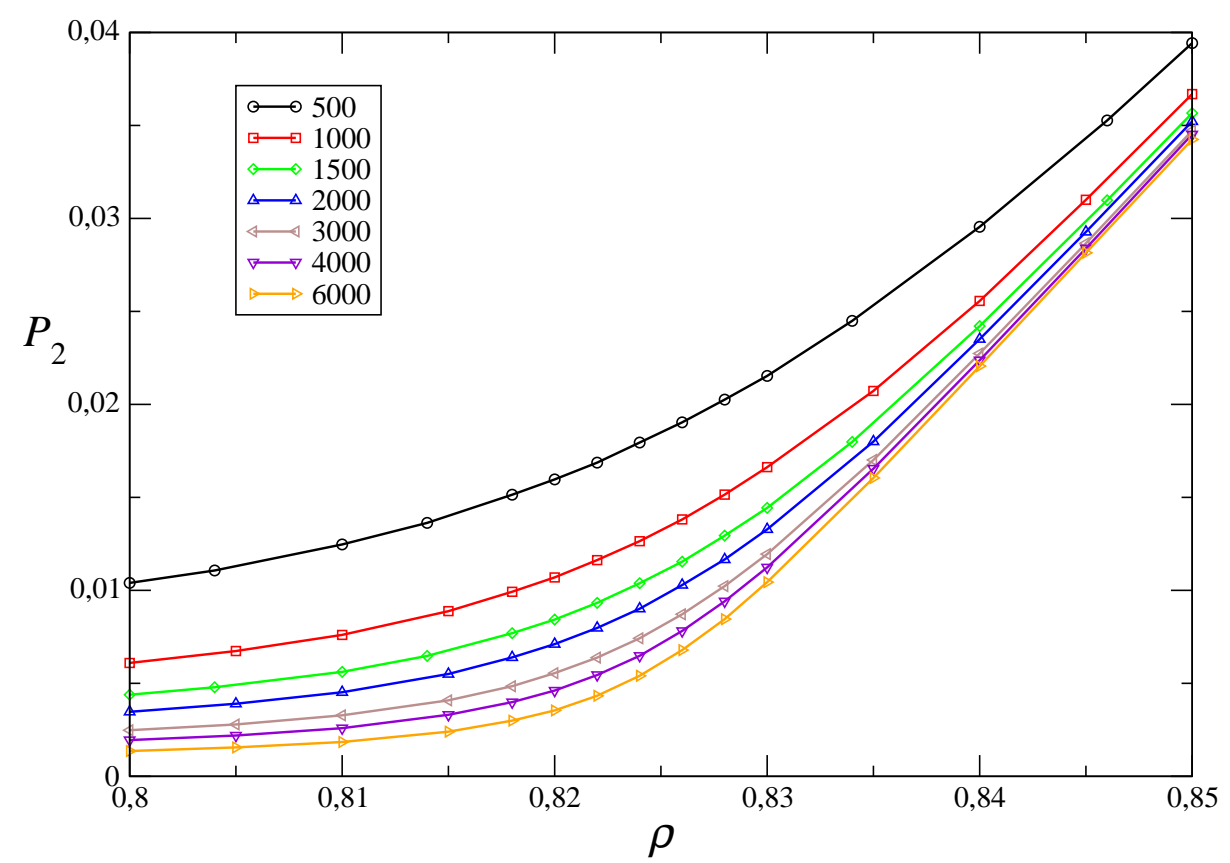

Figura 3.12: Gráfico $P_{2} \times \rho$ para diversos valores de $L$, nas vizinhanças do ponto crítico, para $p=0.4$. Para este ponto, estimamos $\rho_{c}=0,822(2)$.

flutuações nas diversas densidades de aglomerados diminuem após esse passo, e a densidade de partículas mantém-se constante durante a atualização.

Podemos ver como a densidade de sítios ativos varia com $L$ perto do ponto crítico na figura 3.12. Para $L=500$, o menor valor possível de $P_{2}$ é igual a 0,002 (um sítio ativo), de modo que $P_{2}$ deve ser maior ou igual a este valor, não importa o quanto abaixo do ponto crítico (estimado por métodos mostrados adiante como sendo 0,822(2)) o sistema esteja. De fato, o valor médio de $P_{2}$ será sempre maior que 0,002 , devido às flutuações sofridas por $P_{2}$. Conforme $L$ aumenta, o valor mínimo de $P_{2}$ diminui, e o valor médio dessa grandeza aproxima-se de zero para $\rho \leq \rho_{c}$.

Para o cálculo da densidade crítica para cada valor de $p$, utilizamos três métodos: o método da razão de momentos, o da linearização do gráfico de $P_{2} \times L$ e o da linearização do gráfico de $P_{2} \times\left|\rho-\rho^{*}\right|$. Cada um dos moétodos é discutido rapidamente a seguir.

Para o primeiro método, definimos os momentos do parâmetro de ordem $P_{2}[5]$ :

$$
\mu_{n}=\left\langle P_{2}^{n}\right\rangle
$$


O primeiro momento de $P_{2}$ é simplesmente seu valor médio. A partir de argumentos de teoria de escala finita, pode-se mostrar (ver Binder [27], Dickman e Kamphorst L. da Silva [29]) que razões entre momentos de ordens diferentes da forma $\mu_{n} / \mu_{r}^{i} \mu_{s}^{j}$ devem ser independentes do tamanho da rede e das regras do modelo no ponto crítico, desde que $i r+j s=n$.

Escolhemos calcular a razão

$$
m=\frac{\mu_{2}}{\mu_{1}^{2}}=\frac{\mu_{2}}{\left\langle P_{2}\right\rangle^{2}}
$$

que satisfaz o critério acima, para sistemas com diversos valores de $L$, entre 500 e 6000 sítios, e diversos valores de $\rho$ e de $p$ perto do ponto crítico estimado pela aproximação de campo médio de trincas para cada $p$. Fazendo um gráfico, para cada $p$, de $m \times \rho$, percebemos que as curvas obtidas para cada $L$ se cruzam aproximadamente no mesmo ponto, como podemos ver na figura $3.13 \mathrm{a}$, obtida tomando $p=0,49$.

Para obter a densidade crítica a partir dos gráficos $m \times \rho$, foi usado o seguinte procedimento:

- Utilizamos splines (uma categoria de aproximações polinomiais, ver Burden e Faires [26]) para interpolar as curvas $m(\rho)$ dentro do intervalo de dados;

- A seguir, calculamos o ponto de intersecção $\rho_{L / L^{\prime}}$ entre a curva referente ao tamanho $L$ e a curva referente ao tamanho $L^{\prime}$ imediatamente superior a $L$;

- Fizemos outro gráfico, de $\rho_{L / L^{\prime}} \times \ln L$, para estimar o comportamento dos pontos de intersecção conforme $L$ cresce. O ponto de intersecção tende a se estabilizar conforme $L$ cresce (uma vez que o erro inerente a cada ponto deve diminuir conforme $L$ aumenta). A figura 3.13b ilustra esse cálculo.

- A partir dos pontos referentes aos maiores tamanhos, estimamos $\rho_{c}$ e sua incerteza.

Observando a figura 3.13b, nota-se que é difícil determinar a incerteza da densidade crítica calculada por este método. Assim, utilizando os resultados obtidos pelo primeiro método como primeiras aproximações, calculamos $\rho_{c}$ para cada $p$ pelo método de linearização do gráfico $\rho \times L$, explicado a seguir.

Assumindo que a hipótese de escala (3.91) é verdadeira, a função de escala $\mathcal{F}\left(\epsilon L^{1 / \nu_{\perp}}\right)$ deve ser igual a uma constante no ponto crítico (uma vez que $\epsilon=\rho-\rho_{c}=$ 

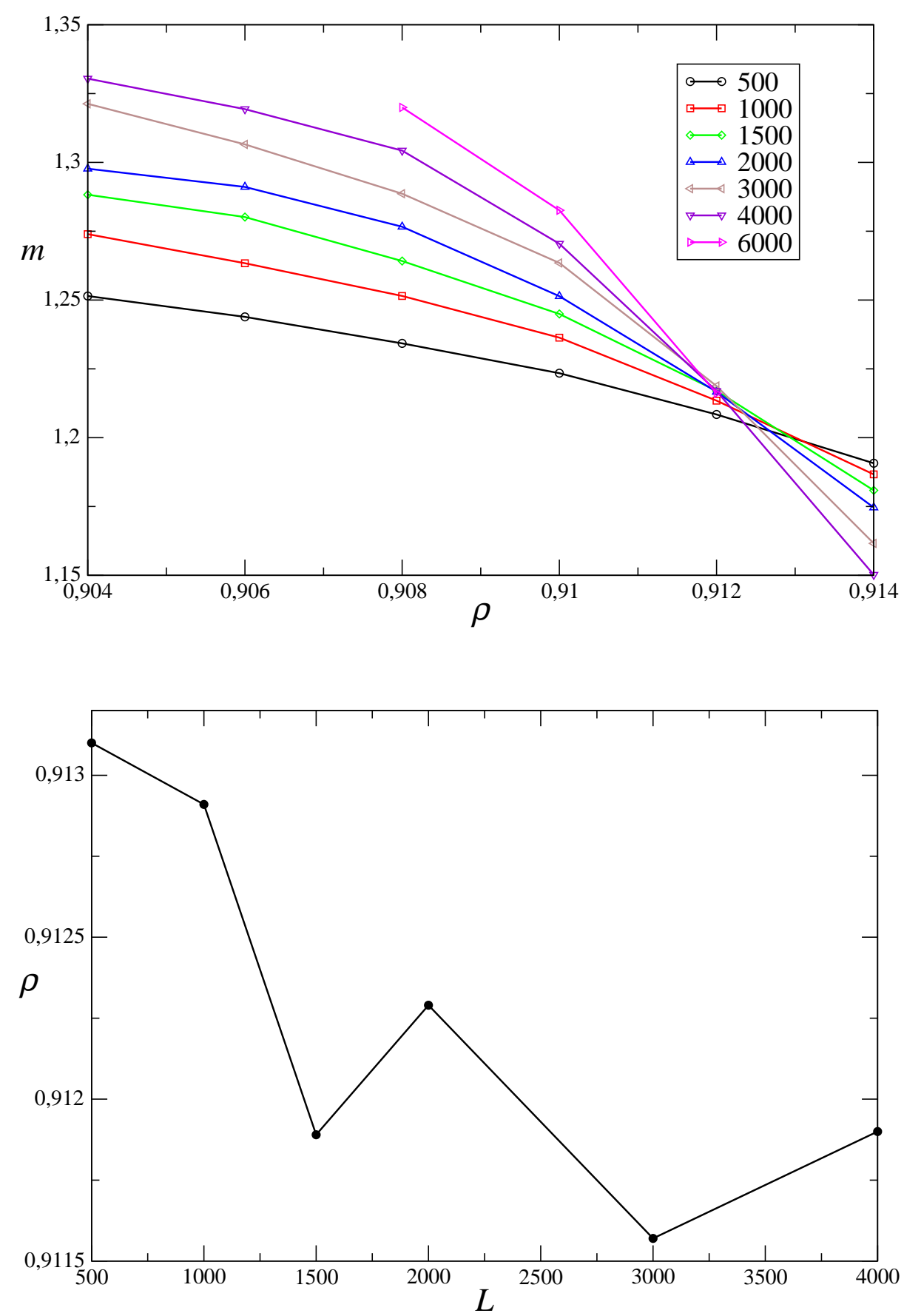

Figura 3.13: (a) Gráfico $m \times \rho$ para diversos valores de $L$ e $p=0,49$. As retas ligando cada par de pontos servem para melhorar a legibilidade do gráfico, separando conjuntos de dados. (b) Gráfico $\rho_{L / L^{\prime}} \times L$, obtido a partir dos pontos de intersecção entre splines utilizados para interpolar as curvas correspondentes aos tamanhos $L$ e $L^{\prime}$ (tamanho imediatamente superior a $L$ ) do gráfico anterior. 


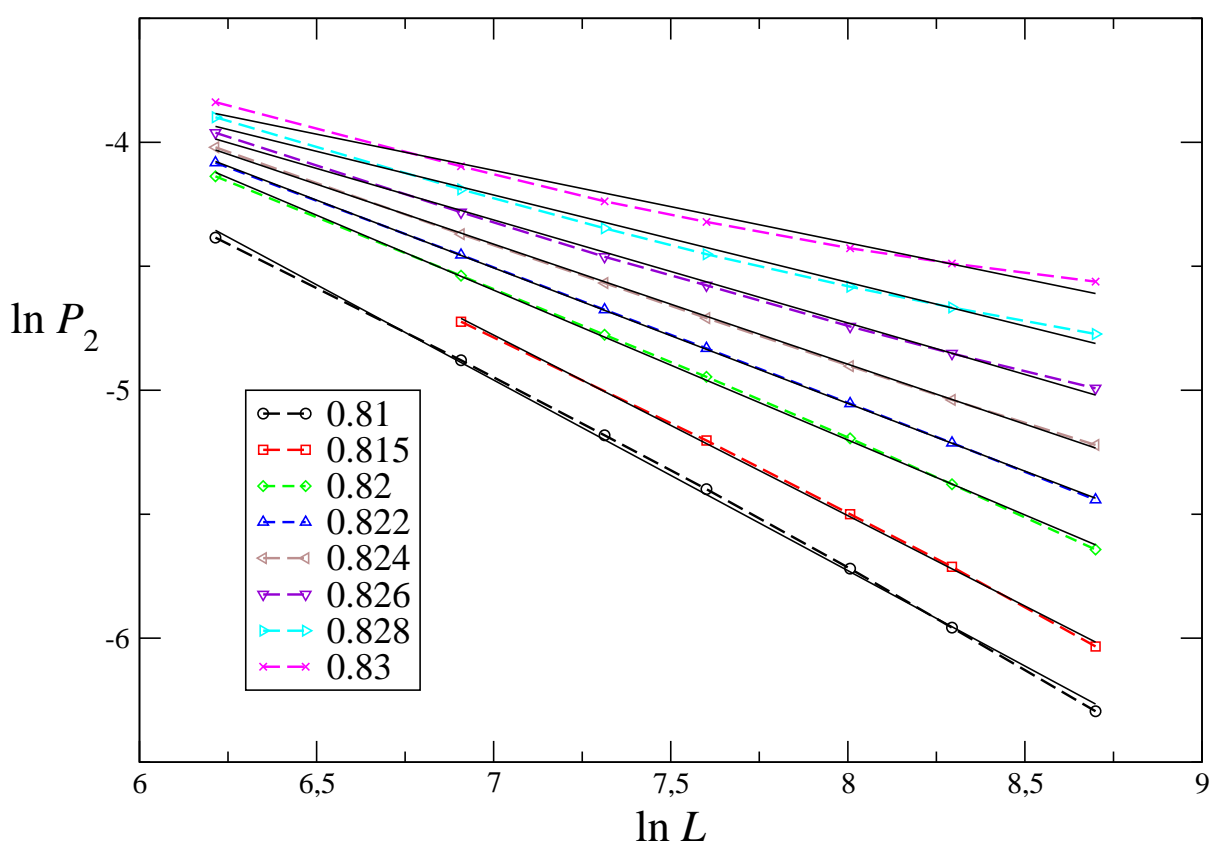

Figura 3.14: Gráfico $\ln \rho_{a} \times \ln L$ para vários valores de $\rho$ e $p=0,4$ (linhas tracejadas) e retas ajustadas a cada gráfico (linhas contínuas). Obtemos $\rho_{c}=0,822(2)$ e $\beta / \nu_{\perp}=$ $0,55(5)$.

0), de modo que podemos linearizar essa expressão tomando o logaritmo de cada lado:

$$
\ln P_{2}=-\frac{\beta}{\nu_{\perp}} \ln L+\ln \mathcal{F}(0)
$$

Assim, quando $\rho=\rho_{c}$, o gráfico de $\ln P_{2} \times \ln L$ deve ser uma reta de inclinação $-\beta / \nu_{\perp}$; outros valores de $\rho$ devem produzir gráficos com curvatura, devido à variação do argumento de $\mathcal{F}$.

Conhecendo-se a região onde o ponto crítico está localizado, pelo método anterior, para cada valor de $p$, podemos simular o sistema para vários valores de $\rho$ nas redondezas do ponto crítico e para vários valores de $L$ (no mínimo 5 valores distintos) e obter o gráfico de $\ln P_{2} \times \ln L$, para cada $\rho$. O gráfico que for melhor ajustado por uma reta (ou seja, aquele cujos coeficientes da reta ajustada têm as menores incertezas) fornecerá nossa medição de $\rho_{c}$, com uma incerteza dada pela diferença entre os dois valores mais próximos de $\rho$. A figura 3.14 ilustra o cálculo de $\rho_{c}$ por este método.

Este método fornece, além da estimativa de $\rho_{c}$, uma estimativa de $\beta / \nu_{\perp}$ igual ao módulo do coeficiente angular, e uma estimativa da incerteza de $\beta / \nu_{\perp}$, calcu- 


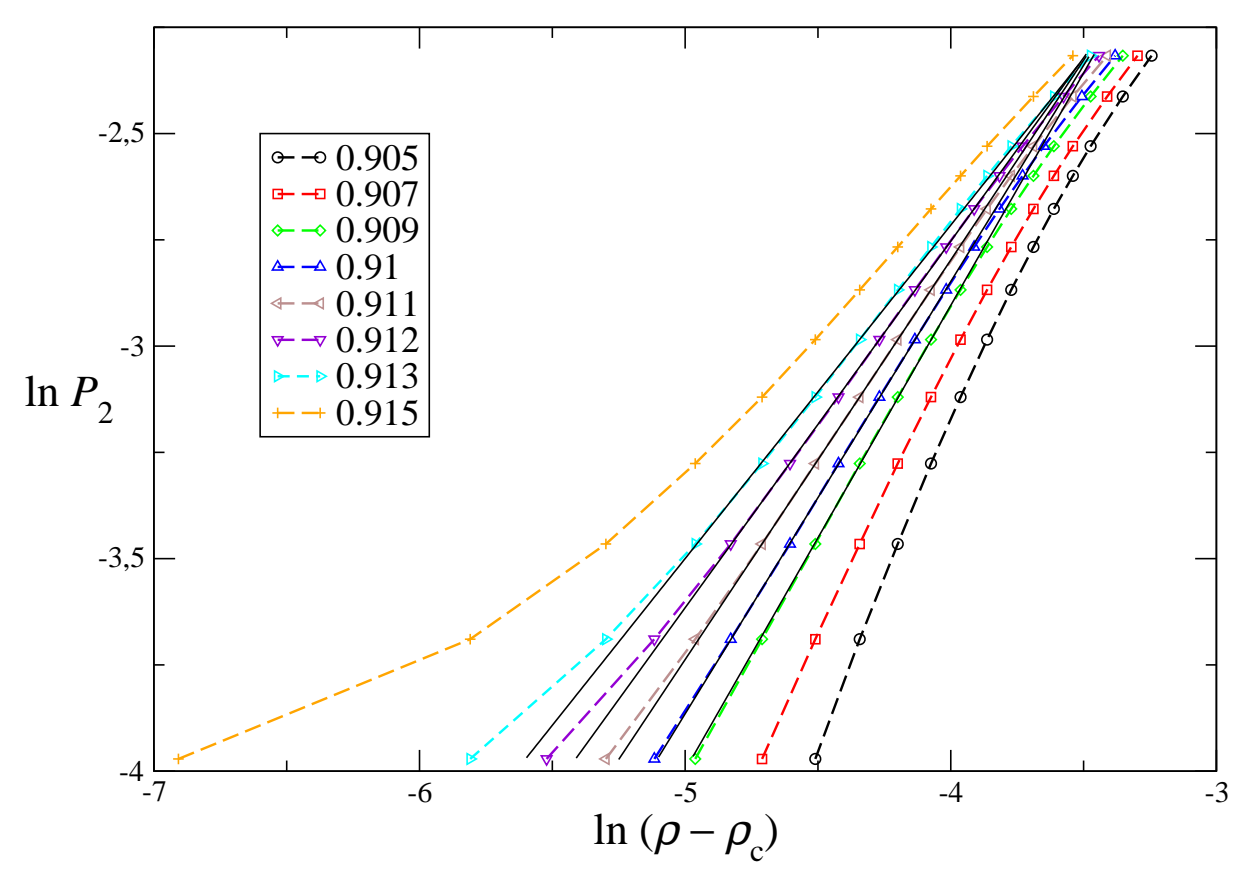

Figura 3.15: Gráfico $\ln \rho_{a} \times \ln \left|\rho-\rho_{c}\right|$ para $\mathrm{L}=4000, p=0,49$ (linhas tracejadas) e ajuste de reta calculado no intervalo $0,92 \leq \rho \leq 0,928$ (linhas contínuas). O ponto crítico é $\rho_{c}=0,911(1)$ e o expoente crítico é $\beta=0,94(5)$.

lada como a diferença média entre o coeficiente angular da reta correspondente à densidade crítica e o das retas vizinhas mais próximas.

Um terceiro método também pode ser utilizado para medir a densidade crítica. Tomando o logaritmo da equação (3.27), que define o expoente crítico $\beta$, obtemos:

$$
\ln P_{2}=\beta \ln \left|\rho-\rho_{c}\right| \quad \text { no limite } L \rightarrow \infty
$$

relação que pode ser utilizada para obter uma nova estimativa de $\rho_{c}$, bem como uma medida direta do expoente $\beta$.

Para obter $\rho_{c}$ com este método, mantemos $L$ fixo e construímos gráficos de $\ln P_{2} \times \ln \left|\rho-\rho_{c}\right|$ para diversos valores $\rho_{c}$. Tais gráficos possuem três regiôes distintas: para $\left|\rho-\rho_{c}\right|$ muito grande, a relação (3.27) não é mais válida devido ao aparecimento de termos de correção da forma $\left(\rho-\rho_{c}\right)^{v}, v<\beta$. Na região central, o gráfico é linear e, no limite $\left|\rho-\rho_{c}\right| \rightarrow 0$, ele deixa de ser linear devido às correções incluídas no modelo para que ele não caia no estado absorvente. Como a influência dessas correções é menor quando o tamanho do sistema é maior, devemos usar conjuntos de dados com $L$ grande para que a região central seja mais extensa e possamos estimar 
Tabela 3.4: Densidade crítica $\rho_{c}$, expoentes críticos $\beta$ e $\nu_{\perp}$ e razão de momentos $m$ no ponto crítico em função do parâmetro $p$. As últimas duas linhas são resultados para a versão simétrica obtidos por Dickman, Tomé e Oliveira [12] e por Dickman [18], respectivamente.

\begin{tabular}{|c|c|c|c|c|}
\hline$p$ & $\rho_{c}$ & $\beta / \nu_{\perp}$ & $\beta$ & $m$ \\
\hline 0,01 & $0,56(1)$ & $0,45(2)$ & $1,08(8)$ & $1,23(3)$ \\
0,1 & $0,634(1)$ & $0,53(2)$ & $1,07(4)$ & $1,220(5)$ \\
0,2 & $0,687(3)$ & $0,50(2)$ & $1,09(9)$ & $1,233(4)$ \\
0,3 & $0,748(3)$ & $0,50(2)$ & $1,07(7)$ & $1,238(4)$ \\
0,4 & $0,820(3)$ & $0,54(3)$ & $1,04(5)$ & $1,238(5)$ \\
0,49 & $0,910(1)$ & $0,61(5)$ & $1,0(1)$ & $1,214(7)$ \\
\hline 0,5 & $0,92965(3)$ & $0,247(2)$ & $0,412(4)$ & $1,1596(4)$ \\
0,5 & $0,929780(7)$ & $0,213(6)$ & $0,289(13)$ & $1,142(8)$ \\
\hline
\end{tabular}

$\rho_{c}$ com uma incerteza menor.

A figura 3.15 mostra como podemos calcular $\rho_{c}$ e $\beta$. A partir de um conjunto de dados para $p=0,49, L=4000$ sítios e $0,916 \leq \rho \leq 0,944$ (acima do ponto crítico estimado por outros métodos), construímos os gráficos $\ln P_{2} \times \ln \left|\rho-\rho_{c}\right|$ para diversos valores de $\rho_{c}$ e ajustamos retas à região central do gráficos pelo método de mínimos quadrados. A reta que melhor se ajusta ao conjunto de dados correspondente fornece o valor $\rho_{c}=0,911$ para o ponto crítico, tendo sido obtida por ajuste de reta entre $\rho=0,92$ e $\rho=0,928$. Para $\rho \geq 0,93$, surgem correções à expressão (3.27) e, para $\rho<0,92$, o sistema está perto o suficiente da transição para o estado absorvente para que a mudança nas regras do modelo afete o resultado.

Repetimos o processo descrito acima para obter a densidade crítica e os expoentes críticos $\beta$ e $\nu_{\perp}$ para vários valores de $p$ entre 0 e 0,5 . Os resultados obtidos para os expoentes críticos aparecem na tabela 3.4 , bem como o valor da razão de momentos $m$ no ponto crítico.

A partir dos dados da tabela 3.4, podemos inferir que os expoentes críticos $\beta$ e $\nu_{\perp}$ assumem valores fixos no intervalo $0<p<0,5$, a saber, $\beta=1$ e $\nu_{\perp}=2$ (comparando as flutuações entre dados com suas incertezas) e sofrem uma descontinuidade em $p=0,5$, onde eles assumem os valores descritos nas referências [12, 18] (embora não exista consenso absoluto sobre o valor de $\beta$, vemos que seu valor para a versão simétrica é diferente do nosso valor para a versão assimétrica). Assim, vemos que a assimetria imposta ao modelo de pilha de areia é um elemento relevante do modelo. 


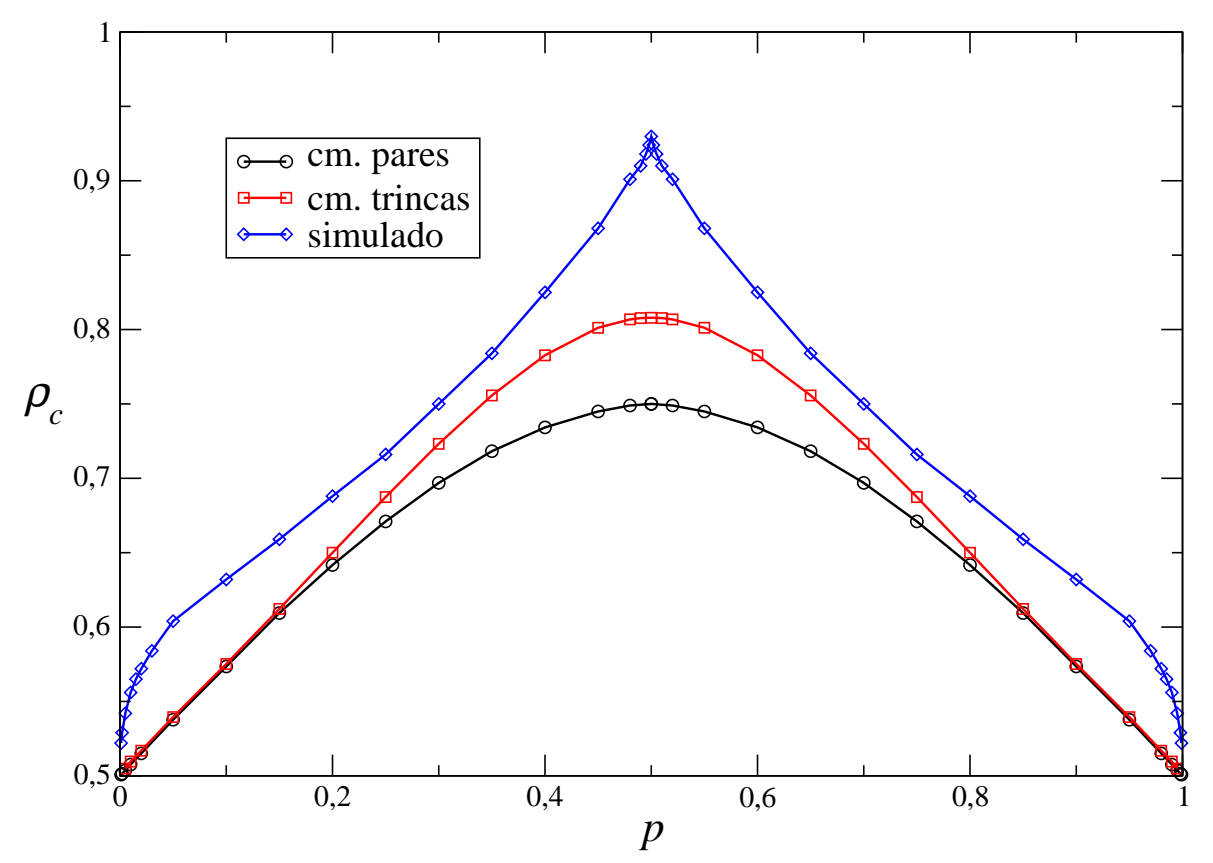

Figura 3.16: Gráfico $\rho_{c} \times p$, obtido por aproximações de campo médio de pares e de trincas e por simulação numérica. O ponto $p=0,5$ da curva por simulação corresponde ao resultado de Dickman [18].

Tendo obtido a densidade crítica em função de $p$ por cada um dos métodos, calculamos um valor médio para $\rho_{c}(p)$ (que depende das incertezas estimadas em cada método). O gráfico obtido pode ser visto na figura 3.16, onde também colocamos os resultados obtidos pelas aproximações de campo médio de pares e de trincas, para fins de comparação.

Analisando a figura 3.16, vemos que os resultados obtidos por simulação são qualitativamente diferentes das aproximações de campo médio: nos limites $p \rightarrow 0$ ou 1 , as aproximações de campo médio mostram que $\rho_{c}$ como uma função linear de $p$, com derivada finita, enquanto o resultado da simulação aponta para uma função com derivada infinita em $p=0$ ou 1 . Podemos assumir que a forma funcional de $\rho_{c}(p)$ é uma lei de potência:

$$
\rho_{c}(p)-\rho_{c}\left(p_{0}\right)=A\left|p-p_{0}\right|^{\mu}, \quad p_{0}=0,1, \quad \rho_{c}\left(p_{0}\right)=0,5 .
$$

Tomando o logaritmo natural de cada lado e ajustando uma função linear por mínimos quadrados, obtemos $A=0,34(4)$ e $\mu=0,396(3)$.

$\operatorname{Em} p=0,5, \rho_{c}(p)$ também exibe uma descontinuidade na primeira derivada, ao 
contrário das aproximações de campo médio, que exibem a primeira derivada nula nesse ponto. Assumindo novamente uma lei de potência nessa região:

$$
\left|\rho_{c}(p)-\rho_{c}(0,5)\right|=B|p-0,5|^{\mu^{\prime}}, \quad \rho_{c}(0,5)=0,92978
$$

obtemos $B=0,590(10), \mu^{\prime}=0,742(4)$.

\subsubsection{Fluxo de partículas}

Realizamos simulações do modelo de pilha de areia assimétrico tomando valores de $p$ e $\rho$ espalhados uniformemente dentro do intervalo de definição das grandezas, usando uma rede de 1000 sítios, de modo a obter estimativas numéricas para $\phi$ em função de $p$ e $\rho$.

Utilizando os resultados da simulação, podemos calcular $\phi$ diretamente, contando o número de partículas que se movimentaram para a direita e subtraindo o número de partículas que se movimentaram para a esquerda em uma unidade de tempo (um passo de Monte Carlo) e dividindo o resultado por $L$. Uma vez que o fluxo medido depende da unidade de tempo escolhida e, durante o processo de simulação, o intervalo de tempo efetivo entre uma tentativa de atuallização e outra não é constante, mas depende do valor instantâneo de $P_{2}$ (uma vez que estamos escolhendo sítios a partir de uma lista de sítios ativos, e não de forma aleatória na rede, de forma a escolher sempre sítios ativos), é necessário multiplicar este resultado por $P_{2}$ para obter o valor atual de $\phi$.

Simulamos o sistema utilizando 1000 condições iniciais diferentes e obtendo a média de $\phi$ no estado estacionário sobre as realizações, para vários valores de $p$ e $\rho$. Os resultados obtidos aparecem na figura 3.17, juntamente com resultados da aproximação de campo médio de pares e de trincas.

Analisando essa figura, vemos que, para baixas densidades, o fluxo obtido por simulação é menor do que o calculado pelas aproximações de campo médio. Da mesma forma que obtemos valores de $\rho_{c}$ mais próximos aos da simulação usando a aproximação de trincas que a aproximação de pares, a aproximação de trincas também produz um fluxo numericamente mais próximo ao da simulação. Entretanto, um resultado notável é observado na região de altas densidades: a aproximação de campo médio de pares produz resultados mais próximos aos da simulação que a aproximação de trincas. É possível que este resultado tenha origem no fato, já 


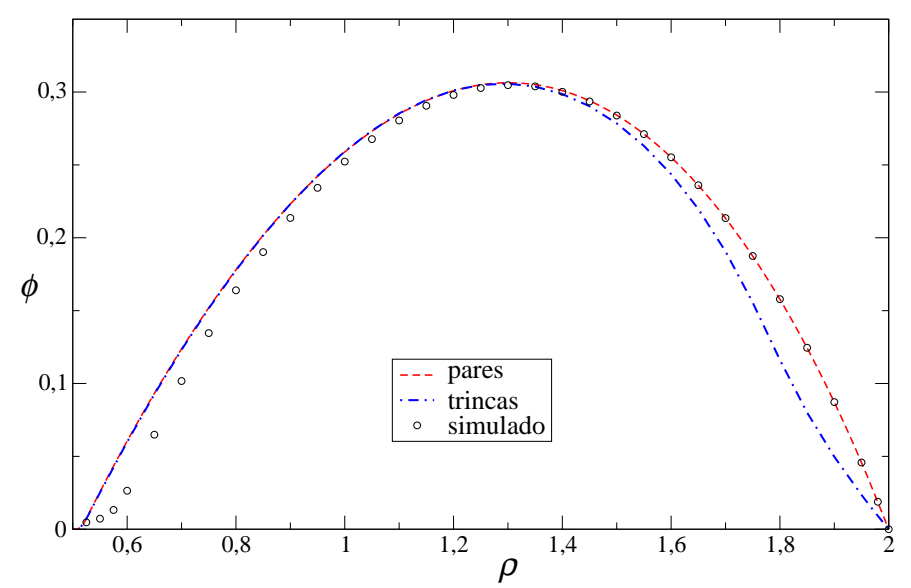

(a) $p=0,02$

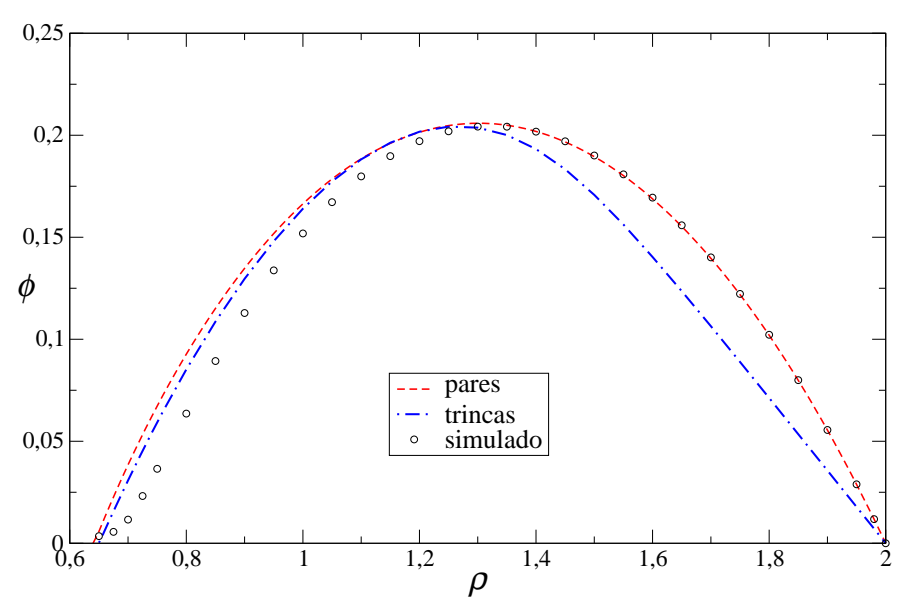

(b) $p=0,2$

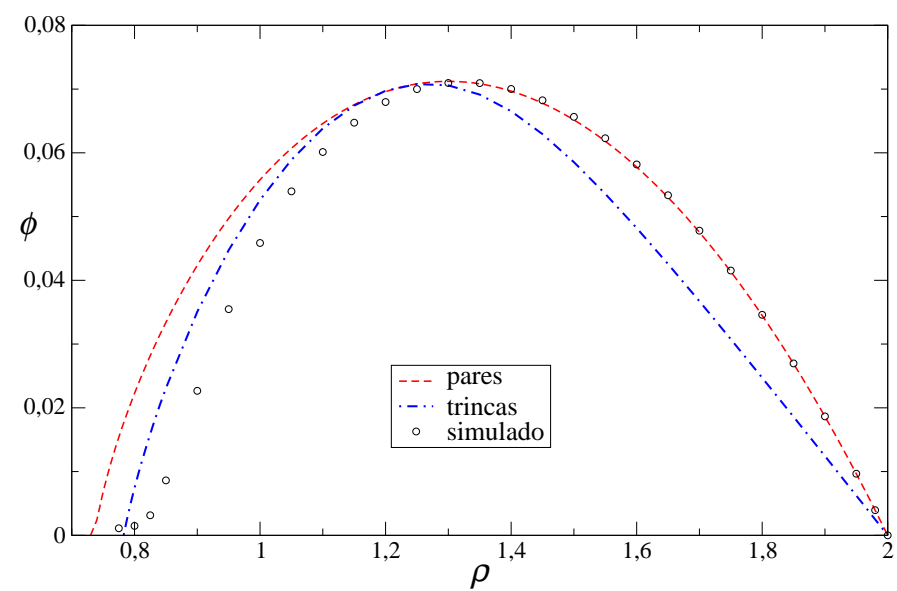

(c) $p=0,4$

Figura 3.17: Fluxo de partículas calculado por simulação e por aproximações de campo médio de pares e de trincas, para alguns valores de $p$. 
notado no capítulo 3.4, de que o conjunto de equações envolvidas no cálculo da aproximação de campo médio é muito mais complexo e instável quando tentamos integrá-lo numericamente pelo método de Euler. Assim, é possível que o estado estacionário não possa ser reproduzido com exatidão suficiente, utilizando o método de Euler.

Observamos que o fluxo numérico não apresenta descontinuidades em $p$ ou em $\rho$. Quando a densidade se aproxima de seu valor crítico, observamos que $\phi$ aparenta não ter dependência linear $\operatorname{com} \rho$ : na verdade, este é outro efeito provocado pela implantação em nosso programa da regra que cria um novo estado ativo sempre que o sistema cai no estado estacionário, a qual é ativada mais frequentemente perto do ponto crítico. Simulando o sistema em uma rede de tamanho maior, observase uma diminuição deste efeito, embora não seja possível fazer com que esse efeito desapareça sem um enorme gasto de tempo e poder computacional. 


\section{Capítulo 4}

\section{O modelo de Glauber linear}

O modelo de Ising [21] é um dos modelos estatísticos mais simples capazes de descrever o comportamento de materiais magnéticos em função da temperatura e sua resposta a um campo magnético externo. Nesse modelo, cada átomo é representado por uma variável de spin $\sigma_{i}$ que pode assumir os valores \pm 1 . Dois spins próximos interagem, de modo que sua energia é menor quando eles possuem o mesmo sentido do que quando possuem sentidos opostos. A temperaturas altas, o sistema deve apresentar-se num estado desordenado, com spins descorrelacionados, enquanto a temperaturas baixas, deve existir um estado preferencial para os spins. A energia do sistema de Ising é definida como:

$$
E(\sigma)=-\frac{J}{2} \sum_{i} \sum_{\delta} \sigma_{i} \sigma_{i+\delta}-H \sum_{i} \sigma_{i}
$$

onde a soma sobre $\delta$ representa uma soma sobre os primeiros vizinhos do sítio $i, J$ é uma constante que mede a interação entre spins próximos e $H$ é uma constante proporcional ao campo magnético externo.

Ao apresentar este modelo, E. Ising previu que ele não apresentaria transição de fase para um estado ordenado a temperatura não nula em nenhuma dimensão. Em 1944, L. Onsager [22] mostrou analiticamente que o modelo de Ising apresenta uma transição de fase entre um estado ordenado e um estado desordenado a uma temperatura finita em 2 dimensões, contrariando o argumento de Ising (embora seu raciocínio estivesse essencialmente correto para $d=1$ ).

O modelo de Glauber, discutido pela primeira vez por R. Glauber [20], é um modelo dinâmico cujo estado estacionário corresponde a um sistema de Ising em 
equilíbrio com um banho térmico a uma tempertura constante. Nesse modelo, uma configuração $\sigma$ evolui pela inversão do valor da variável de spin $\sigma_{i}$, com uma taxa dependente do valor dos spins mais próximos do sítio $i$.

A evolução temporal do modelo de Glauber é regida pela equação mestra:

$$
\frac{d}{d t} P(\sigma, t)=\sum_{\sigma^{\prime} \neq \sigma} W\left(\sigma, \sigma^{\prime}\right) P\left(\sigma^{\prime}, t\right)-W\left(\sigma^{\prime}, \sigma\right) P(\sigma, t)
$$

onde $W\left(\sigma^{\prime}, \sigma\right)$ é a taxa de transição da configuração $\sigma$ para a configuração $\sigma^{\prime}$. Para o modelo de Glauber,

$$
\begin{aligned}
W\left(\sigma^{\prime}, \sigma\right) & =\sum_{i} w_{i}(\sigma), \quad \text { com } \\
w_{i}(\sigma) & =\frac{\alpha}{2}\left\{1-\sigma_{i} \tanh \left[K \sum_{\delta} \sigma_{i+\delta}\right]\right\}
\end{aligned}
$$

onde $\alpha$ é uma constante ligada à escala temporal do modelo.

A taxa de inversão (4.4) satisfaz o critério de balanceamento detalhado

$$
w_{i}\left(\sigma^{i}\right) P\left(\sigma^{i}\right)=w_{i}(\sigma) P(\sigma)
$$

para a probabilidade estacionária:

$$
P(\sigma)=\frac{1}{Z} \exp \left\{\sum_{i} \sum_{\delta} \sigma_{i} \sigma_{i+\delta}\right\}
$$

que é a probabilidade estacionária de um sistema de Ising em equilíbrio a uma temperatura $T=J / 2 k_{B} K$. O critério de balanceamento detalhado implica que, no equilíbrio, o número médio de transições $\sigma \rightarrow \sigma^{\prime}$ é igual ao de transições $\sigma^{\prime} \rightarrow \sigma$, no final de um intervalo longo de tempo.

Em uma dimensão, a taxa de transição do modelo de Glauber pode ser reescrita na forma:

$$
w_{i}(\sigma)=\frac{\alpha}{2}\left\{1-\frac{\lambda}{2} \sigma_{i} \sum_{\delta} \sigma_{i+\delta}\right\}
$$

com $\lambda=\tanh 2 K$. Assim, vemos que o modelo de Glauber, em uma dimensão, possui uma taxa de transição linear nas variáveis $\sigma_{i+\delta}$. Em duas ou mais dimensões, não é possível reescrever (4.4) utilizando apenas termos lineares nos spins vizinhos.

$\mathrm{Na}$ maior parte deste capítulo, vamos estudar a versão linear do modelo de 
Glauber, que consiste em utilizar a taxa de transição (4.7) em vez de (4.4), para qualquer dimensão $d$. O modelo de Glauber linear (modelo GL) pode ser resolvido de forma analítica escrevendo a solução formal da equação mestra na forma matricial como uma expansão em série perturbativa do parâmetro $\lambda$. Na última seção, veremos como adaptar o método de expansão em série para o modelo de Glauber em duas dimensões, situação na qual não é mais possível obter uma forma analítica para as grandezas dinâmicas do modelo.

\subsection{O método de expansão em série perturbativa}

Nesta seção, vamos expor brevemente o método de expansão em série de potências perturbativa para resolução da equação mestra, como descrito por Tomé e de Oliveira [5]. Primeiramente, vamos mostrar como a equação mestra pode ser reescrita na forma matricial, e algumas propriedades úteis nesta representação.

\subsubsection{Matriz de evolução}

Como comentado anteriormente, a equação mestra de um modelo estocástico pode ser reescrita como uma equação matricial. Na expressão (4.2), o termo $W(\sigma, \sigma)$, que representa a taxa de transição entre uma configuração $\sigma$ e ela mesma, não aparece, de modo que podemos defini-lo como quisermos. Uma definição bastante útil, que permite transformar (4.2) numa equação matricial, é:

$$
W(\sigma, \sigma)=-\sum_{\sigma^{\prime}(\neq \sigma)} W\left(\sigma^{\prime}, \sigma\right)
$$

Utilizando a convenção acima, a equação mestra pode ser reescrita como:

$$
\frac{d}{d t} P(\sigma, t)=\sum_{\sigma^{\prime}} W\left(\sigma, \sigma^{\prime}\right) P\left(\sigma^{\prime}, t\right)
$$

E, definindo $|P(t)\rangle$ como um vetor cujas entradas são iguais a $P(\sigma, t)$, reescrevemos a equação acima como:

$$
\frac{d}{d t}|P(t)\rangle=W|P(t)\rangle
$$

onde $W$ é a matriz de evolução associada ao processo estocástico, cujas entradas são iguais aos termos $W\left(\sigma, \sigma^{\prime}\right)$. 
Nesta representação, o vetor $|P(t)\rangle$ representa qualquer configuração possível ou combinação de configurações com probabilidades iguais a $P(\sigma, t)$. Entretanto, para que $|P(t)\rangle$ corresponda a um estado possível, é necessário que todas as suas componentes sejam não-negativas e que ele seja normalizado (a soma de todas as componentes deve ser unitária). Em particular, uma configuração $\sigma$ é representada por um vetor $|\sigma\rangle$ com uma única componente não-nula igual a 1.

Utilizando o formalismo da matriz de evolução, podemos escrever uma solução formal para a equação mestra:

$$
|P(t)\rangle=\exp \{t W\}|P(0)\rangle=\left\{I+t W+\frac{t^{2}}{2 !} W^{2}+\frac{t^{3}}{3 !} W^{3}+\ldots\right\}|P(0)\rangle
$$

Esta expansão formal pode ser utlizada para estudar analiticamente modelos com um número finito de estados (ou seja, modelos cuja matriz $W$ possua dimensão pequena), tais como modelos definidos em uma rede com um número pequeno de sítios. Entretanto, à medida que o número de sítios aumenta, o número de estados possíveis tende a aumentar exponencialmente, de modo que é necessário utilizar outros métodos.

Seja $\left\{\Lambda_{k}\right\}$ o conjunto de autovalores da matriz $W$, indexado pela variável $k$. A cada autovalor, podemos associar o autovetor à esquerda $\left\langle\phi_{k}\right|$ e o autovetor à direita $\left|\psi_{k}\right\rangle$, ambos normalizados:

$$
\begin{aligned}
& W\left|\psi_{k}\right\rangle=\Lambda_{k}\left|\psi_{k}\right\rangle \\
& \left\langle\phi_{k}\right| W=\Lambda_{k}\left\langle\phi_{k}\right|
\end{aligned}
$$

Qualquer vetor coluna $|\Psi\rangle$ pode ser escrito como uma combinação linear dos autovetores à direita de $W$ e qualquer vetor linha $\langle\Phi|$ pode ser escrito como uma combinação linear dos autovetores à esquerda de $W$ :

$$
\begin{aligned}
& |\Psi\rangle=\sum_{k} m_{k}\left|\psi_{k}\right\rangle \\
& \langle\Phi|=\sum_{k} m_{k}^{\prime}\left\langle\phi_{k}\right|
\end{aligned}
$$

Dada a definição da matriz $W$, é possível provar que seus autovalores e autovetores possuem algumas propriedades:

- A parte real de qualquer autovalor de $W$ é negativa ou nula. Em particular, 
$W$ possui um autovalor nulo;

- O autovetor correspondente ao autovalor nulo possui componentes não-negativas;

Se uma configuração qualquer $\sigma$ puder ser alcançada a partir de qualquer outra configuração $\sigma^{\prime}$, as condições acima podem ser escritas de modo mais restrito:

- $W$ possui um autovalor nulo não-degenerado (não existem dois autovetores linearmente independentes correspondentes a este autovalor). Os demais autovetores possuem parte real estritamente negativa;

- Todas as componentes do autovetor correspondente ao autovalor nulo são estritamente positivas.

Supondo que as condições acima são verdadeiras, vamos tomar $\Lambda_{0}=0$ como o autovalor nulo, e $\left\langle\phi_{0}\right|$ e $\left|\psi_{0}\right\rangle$ os autovetores correspondentes. Utilizando a expansão formal (4.11) da solução da equação mestra no limite de tempos longos e a decomposição (4.14), obtemos:

$$
\lim _{t \rightarrow \infty}|P(t)\rangle=\lim _{t \rightarrow \infty} e^{t W}|P(0)\rangle=\lim _{t \rightarrow \infty} \sum_{k} m_{k} e^{t \Lambda_{k}}\left|\psi_{k}\right\rangle=m_{0}\left|\psi_{0}\right\rangle
$$

uma vez que a parte real dos autovalores $\Lambda_{k}, k \neq 0$, é negativa. O significado físico da expressão acima é que $\left|\psi_{0}\right\rangle$ corresponde à probabilidade estacionária $\left|P_{e}\right\rangle$ do modelo associado à matriz $W$ (para que $\left|P_{e}\right\rangle$ esteja corretamente normalizado, $m_{0}=1$ ). Assim, as condições acima implicam que o modelo possui um estado estacionário único, que pode ser alcançado a partir de qualquer condição inicial.

$\mathrm{O}$ autovetor à esquerda $\left\langle\phi_{0}\right|$ também possui uma propriedade especial: impondo a condição de normalização, temos:

$$
\sum_{\sigma}\left|P_{e}\right\rangle=1
$$

Entretanto,

$$
\sum_{\sigma}\left\langle\phi_{0} \mid P_{e}\right\rangle=\left\langle\phi_{0} \mid \psi_{0}\right\rangle=1
$$

de modo que, para que as duas expressões acima sejam verdadeiras, todas as componentes de $\left\langle\phi_{0}\right|$ são iguais a 1 . 


\subsubsection{Expansão em série perturbativa}

Podemos deduzir agora o método de expansão em série perturbativa para um modelo estocástico. Supomos que a matriz de evolução do modelo que estamos estudando possa ser escrita na forma:

$$
W=W_{0}+\lambda V
$$

onde $W_{0}$ é uma matriz de evolução com autovalores $\Lambda_{k}$, autovetores à direita $\left|\psi_{k}\right\rangle$ e à esquerda $\left\langle\phi_{k}\right|$ conhecidos. Em particular, $\Lambda_{0}=0$ e todos os demais autovalores possuem parte real estritamente negativa, de modo que o estado estacionário do modelo descrito pela matriz $W_{0}$ é único.

Nosso objetivo é obter o vetor probabilidade estacionária $\left|P_{e}\right\rangle$ associado à matriz $W$. Vamos supor que ele possa ser escrito como uma expansão em série de potências do parâmetro $\lambda$ :

$$
\left|P_{e}\right\rangle=\left|P_{0}\right\rangle+\sum_{\lambda=1}^{\infty} \lambda^{l}\left|P_{l}\right\rangle
$$

e vamos substituir esta expansão em (4.19). Obtemos:

$$
\left(W_{0}+\lambda V\right)\left(\left|P_{0}\right\rangle+\sum_{\lambda=1}^{\infty} \lambda^{l}\left|P_{l}\right\rangle\right)=W\left|P_{e}\right\rangle=0
$$

Para que a expressão acima seja verdadeira, o coeficiente de cada potência de $\lambda$ deve ser identicamente nulo:

$$
\begin{aligned}
& W_{0}\left|P_{0}\right\rangle=0 \\
& W_{0}\left|P_{l}\right\rangle=-V\left|P_{l-1}\right\rangle, \quad l \geq 1
\end{aligned}
$$

A partir de (4.22), concluímos que $\left|P_{0}\right\rangle=\left|\psi_{0}\right\rangle$, o vetor probabilidade estacionária de $W_{0}$. Usando (4.22) e (4.23), podemos escrever uma relação de recorrência para os termos $\left|P_{l}\right\rangle$. Primeiramente, definimos uma matriz $R$, que comporte-se como a inversa da matriz $W_{0}$ ( $W_{0}$ não possui uma matriz inversa, tendo em vista que um de seus autovalores é nulo). Uma vez que vale a expansão:

$$
W_{0}=\sum_{k(\neq 0)}\left|\psi_{k}\right\rangle \Lambda_{k}\left\langle\phi_{k}\right|
$$


podemos escrever $R$ como:

$$
R=\sum_{k(\neq 0)}\left|\psi_{k}\right\rangle \frac{1}{\Lambda_{k}}\left\langle\phi_{k}\right|
$$

Vemos que o produto entre as matrizes $W_{0}$ e $R$ é:

$$
R W_{0}=W_{0} R=I-\left|\psi_{0}\right\rangle\left\langle\phi_{0}\right|
$$

ou seja, fora do subespaço associado ao vetor $\left|\psi_{0}\right\rangle, R$ é a matriz inversa de $W_{0}$.

Multiplicando (4.23) à esquerda por $R$ e utilizando (4.26), obtemos a relação de recorrência para os termos $\left|P_{l}\right\rangle$ :

$$
\left|P_{l}\right\rangle=-R V\left|P_{l-1}\right\rangle, \quad l \geq 1
$$

Aplicando-se essa equação recursivamente, obtemos a expressão para $\left|P_{e}\right\rangle$ :

$$
\left|P_{e}\right\rangle=\left|\psi_{0}\right\rangle+\sum_{\lambda=1}^{\infty}(-\lambda R V)^{l}\left|\psi_{0}\right\rangle
$$

A principal utilidade deste método não é calcular o vetor probabilidade estacionária, mas calcular valores médios de grandezas físicas, como energia livre ou susceptibilidade. Dada a grandeza $F(\sigma)$, seu valor médio é igual a:

$$
\langle F\rangle=\sum_{\sigma} F(\sigma) P_{e}(\sigma)=\left\langle\phi_{0}|F| P_{e}\right\rangle
$$

onde $\left|P_{e}\right\rangle$ é dado por (4.28).

Na maioria dos problemas de interesse, não é necessário calcular todos os termos da expansão em série de $\left|P_{e}\right\rangle$, mas apenas os termos tais que (4.29) não se anule. Assim, apenas é necessário calcular os termos da expansão cuja imagem pelo operador $F$ é proporcional ao vetor $\left|\psi_{0}\right\rangle$. Devido à presença do operador $F$ em (4.29), é necessário o cálculo dos termos apropriados para cada grandeza física $F$.

\subsection{O modelo GL unidimensional}

Nesta seção, vamos utilizar o método de expansão em série perturbativa para calcular a susceptibilidade magnética a campo nulo do modelo GL em uma dimensão, discutindo em detalhes cada etapa do cálculo. Primeiramente, definimos formal- 
mente o modelo de Glauber linear.

Consideramos uma cadeia com $L$ sítios, com condições periódicas de contorno, e a cada sítio $i$ da cadeia associamos a variável de spin $\sigma_{i}$, que pode assumir os valores \pm 1 . Para atualizar a configuração $\sigma$, escolhemos um sítio $i$ e invertemos o sinal da variável $\sigma_{i}$ de acordo com a taxa de transição:

$$
w_{i}(\sigma)=\frac{1}{2}\left\{1-\frac{\lambda}{2} \sigma_{i} \sum_{\delta} \sigma_{i+\delta}\right\}
$$

onde a soma é feita sobre os primeiros vizinhos do sítio $i$ e $\lambda$ é um parâmetro de controle dentro do intervalo $0 \leq \lambda \leq 1$. As transições possíveis no modelo aparecem na figura 4.1 .

Tomando $\lambda=\tanh 2 K$, obtemos a taxa de transição do modelo de Glauber. O parâmetro de escala temporal $\alpha$ é fixado como 1, uma vez que não estamos interessados em uma propriedade dinâmica.

A susceptibilidade magnética por sítio a campo nulo deste modelo é dada por:

$$
\chi=\frac{1}{N}\left\langle\left(\sum_{i} \sigma_{i}\right)^{2}\right\rangle=\left\langle\sigma_{i} \sum_{j} \sigma_{j}\right\rangle=1+\left\langle\sigma_{i} \sum_{j(\neq i)} \sigma_{j}\right\rangle
$$

Assim, para calcular a susceptibilidade do estado estacionário do modelo de Glauber linear, devemos calcular o valor médio $\left\langle\sigma_{i} \sum_{j(\neq i)} \sigma_{j}\right\rangle$. Este cálculo pode ser feito convertendo o problema para a forma matricial e escrevendo $\chi$ como uma expansão

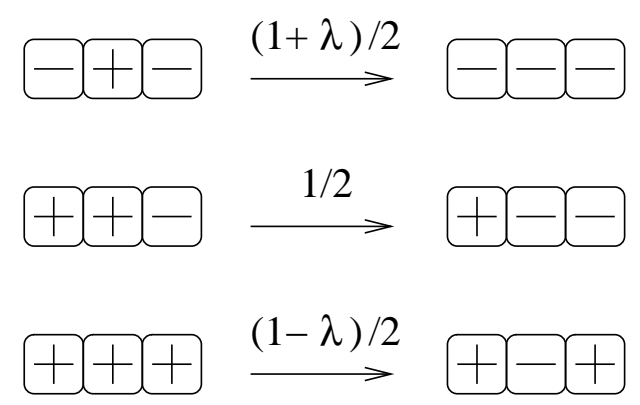

Figura 4.1: Ilustração da taxa de transição para o modelo de Glauber linear - o spin central tem seu valor invertido com taxas dependentes do spin dos primeiros vizinhos. 
em série de potências do parâmetro $\lambda$ :

$$
\chi=\sum_{l=0}^{\infty} \chi^{(l)} \lambda^{l}
$$

\subsubsection{Aplicação do método de expansão em série}

Vamos converter a equação mestra do modelo GL para a forma matricial. Primeiramente, vamos definir alguns operadores, cujo produto por um vetor $|\sigma\rangle$ depende apenas da variável de $\operatorname{spin} \sigma_{i}$ :

$$
\begin{aligned}
I|\sigma\rangle & =|\sigma\rangle \quad \text { (operador identidade) } \\
T_{i}|\sigma\rangle & =\left|\sigma^{i}\right\rangle \quad \text { (operador troca de spin) } \\
M_{i}|\sigma\rangle & =\sigma_{i}|\sigma\rangle \quad \text { (operador magnetização) }
\end{aligned}
$$

onde $\left|\sigma^{i}\right\rangle$ é o vetor obtido de $|\sigma\rangle$ alterando-se o spin do sítio $i$. Com estas definições, o operador $W$ correspondente à equação mestra é:

$$
\begin{aligned}
W & =W_{0}-\frac{\lambda}{2} V=\sum_{i}\left\{S_{i}-\frac{\lambda}{2} V_{i}\right\}, \text { com } \\
S_{i} & =\frac{T_{i}-I}{2} \mathrm{e} \\
V_{i} & =\left(S_{i}+S_{i+1}\right) M_{i} M_{i+1}
\end{aligned}
$$

O operador $W$ já está na forma adequada para a expansão em série de potências do parâmetro $-\lambda / 2$, uma vez que $W_{0}$ é um operador suficientemente simples para ser estudado analiticamente. Assim, podemos aplicar o método de expansão em série perturbativa após conhecermos os autovalores e autovetores de $W_{0}$. A susceptibilidade (4.31) assume a forma:

$$
\chi=1+\left\langle\phi_{0}\left|\sigma_{i} \sum_{j(\neq i)} \sigma_{j}\right| P_{e}\right\rangle=1+\left\langle\phi_{0}\left|M_{i} \sum_{j(\neq i)} M_{j}\right| P_{e}\right\rangle
$$

Começamos a aplicar op método de expansão em série calculando os autovalores e autovetores de $W_{0}$. Primeiramente vamos transformá-lo para a forma diagonal. Para diagonalizar $W_{0}$, que é igual à soma de operadores $S_{i}$ dependentes de um único sítio, podeos diagonalizar cada operador $S_{i}$ separadamente. Cada operador $S_{i}$ pode 
ser visto como o produto externo de operadores $I$ e $S$ que agem em configurações $|s\rangle$ de um único sítio:

$$
S_{i}|\sigma\rangle=(I|s\rangle)_{1} \otimes(I|s\rangle)_{2} \otimes \cdots \otimes(S|s\rangle)_{i} \otimes \cdots \otimes(I|s\rangle)_{L}
$$

Como as matrizes $I$ já estão diagonalizadas, basta obter os autovalores e autovetores de $S$ para diagonalizar $S_{i}$. Sejam

$$
|+\rangle=\left(\begin{array}{l}
1 \\
0
\end{array}\right) \quad|-\rangle=\left(\begin{array}{l}
0 \\
1
\end{array}\right)
$$

os vetores de base para configurações de um único sítio. Nesa base, os operadores $T$ e $S$ assumem as formas:

$$
T=\left[\begin{array}{ll}
0 & 1 \\
1 & 0
\end{array}\right] \quad \text { e } \quad S=\frac{1}{2}\left[\begin{array}{cc}
-1 & 1 \\
1 & -1
\end{array}\right]
$$

Os autovalores do operador $S$ são iguais a 0 e -1 . Os autovetores correspondentes são:

$$
\begin{aligned}
& S\left(\frac{|+\rangle+|-\rangle}{2}\right)=S|0\rangle=0 \\
& S\left(\frac{|+\rangle-|-\rangle}{2}\right)=S|1\rangle=-|1\rangle
\end{aligned}
$$

Assim, a mudança de base $\{|+\rangle,|-\rangle\} \rightarrow\{|0\rangle,|1\rangle\}$ diagonaliza o operador $S$. Para diferenciar as duas bases, vamos utilizar $\eta=0,1$ como rótulos dos vetores da nova base.

Correspondentemente, $W_{0}$ é diagonalizado pela mudança de base $|\sigma\rangle \rightarrow|\eta\rangle$. Os autovalores de $W_{0}$ associados aos vetores $|\eta\rangle$ são: $\Lambda_{k}=-\sum_{i} \eta_{i}=0,-1, \ldots,-N \ldots$ Os autovetores associados a cada autovalor são configurações que possuem $N$ variáveis iguais a 1 e $L-N$ variáveis iguais a 0 .

Utilizando a definição dos vetores $|\eta\rangle$, vemos que $M_{i}|\eta\rangle=\left|\eta^{i}\right\rangle$ : os operadores $M_{i}$, aplicados aos novos vetores base, trocam o valor da variável $\eta_{i}$. Desta forma,

$$
\begin{aligned}
& V_{i}|\ldots 00 \ldots\rangle=-2|\ldots 1 \quad 1 \ldots\rangle \\
& V_{i}|\ldots 10 \ldots\rangle=-|\ldots 01 \ldots\rangle \\
& V_{i}|\ldots 01 \ldots\rangle=-|\ldots 10 \ldots\rangle
\end{aligned}
$$




$$
V_{i}|\ldots 11 \ldots\rangle=0
$$

Acima, apenas as variáveis que ocupam os sítios $i$ e $i+1$ são mostradas; o resultado acima é independente das demais variáveis.

É possível interpretar a mudança de base como definindo um novo modelo de gás de rede. Nele, cada sítio pode ser ocupado por no máximo uma partícula; os autovetores à direita do operador $W_{0}$ são configurações puras com um número $N$ de partículas igual em módulo ao autovalor correspondente, de modo que o estado estacionário $\left|\psi_{0}\right\rangle$ correspondente à matriz $W_{0}$ é a configuração que não possui nenhuma partícula (vácuo). O operador $V$ é igual à soma de operadores que atuam em dois sítios vizinhos: quando os sítios estão vazios, $V_{i}$ cria duas partículas; quando um dos sítios está ocupado, $V_{i}$ transfere a partícula para o sítio vazio e, quando os sítios estão ocupados, $V_{i}$ aniquila o vetor.

Para a demonstração a seguir, vamos adotar uma nova notação: o vetor $|i, j, \ldots k\rangle$ é aquele que possui partículas nos sítios $i, j, \ldots k$, enquanto os demais sítios estão vazios. Conhecendo-se os autovalores e autovetores à direita de $W_{0}$, podemos aplicar o método de expansão em série para determinar os termos $\left|P_{l}\right\rangle$ (relação (4.27)). Aplicando o operador $-R V$ ao vetor $\left|\psi_{0}\right\rangle$, obtemos uma combinação linear de vetores com duas partículas:

$$
\left|P_{1}\right\rangle=-R V\left|\psi_{0}\right\rangle=2 R \sum_{i}|i, i+1\rangle=-\sum_{i}|i, i+1\rangle
$$

Multiplicando (4.48) pelo fator $-\lambda / 2$, obtemos a contribuição de $1^{\mathrm{a}}$ ordem ao vetor $\left|P_{e}\right\rangle$.

Aplicando $-R V$ a (4.48), obtemos dois somatórios distintos: um de vetores com 2 partículas, outro de vetores com 4 partículas:

$$
\begin{aligned}
& -R V\left|P_{1}\right\rangle=R \sum_{j} \sum_{i} V_{j}|i, i+1\rangle= \\
& =R \sum_{i}\left\{V_{i-1}+V_{i}+V_{i+1}+V_{i+2}+\sum_{\substack{j \neq\{i-1, i, i+1, i+2\}}} V_{j}\right\}|i, i+1\rangle \\
& =-R \sum_{i}\left\{|i-1, i+1\rangle+|i, i+2\rangle+2 \sum_{\substack{j \neq\{i-1, i, i+1, i+2\}}}|i, i+1, j, j+1\rangle\right\}
\end{aligned}
$$




$$
\begin{aligned}
& =-2 R \sum_{i}\left\{|i, i+2\rangle+\sum_{\substack{j \neq\{i-1, i, i+1, i+2\}}}|i, i+1, j, j+1\rangle\right\} \\
& =\sum_{i}\left\{|i, i+2\rangle+\frac{1}{2} \sum_{\substack{j \neq\{i-1, i, i+1, i+2\}}}|i, i+1, j, j+1\rangle\right\}
\end{aligned}
$$

Aplicações sucessivas do operador $-R V$ criam vetores com $2,4, \ldots 2 l$ partículas. Veremos a seguir que nem todos estes vetores são necessários para o cálculo da susceptibilidade magnética. De fato, usando a expressão (4.49) para calcular o $2^{\circ}$ termo da expansão de $\chi$ (equação (4.39)), obtemos:

$$
\begin{aligned}
\chi^{(2)} & =\left(-\frac{1}{2}\right)^{2}\left\langle\phi_{0}\left|M_{h} \sum_{h^{\prime}(\neq h)} M_{h^{\prime}}\right| P_{2}\right\rangle \\
& =\left(\frac{1}{2}\right)^{2} \sum_{h^{\prime}(\neq h)} \sum_{i}\left\{\left\langle h, h^{\prime} \mid i, i+2\right\rangle+\frac{1}{2} \sum_{\substack{j \neq\{i-1, i, i+1, i+2\}}}\left\langle h, h^{\prime} \mid i, i+1, j, j+1\right\rangle\right\} \\
& =\left(\frac{1}{2}\right)^{2} \sum_{h^{\prime}(\neq h)}\left\{\left\langle h, h^{\prime} \mid h, h+2\right\rangle+\left\langle h, h^{\prime} \mid h-2, h\right\rangle\right\} \\
& =\left(\frac{1}{2}\right)^{2}\{\langle h, h-2 \mid h, h-2\rangle+\langle h-2, h \mid h-2, h\rangle\}=\frac{1}{2}
\end{aligned}
$$

Do resultado acima, podemos tirar algumas conclusões:

- As únicas parcelas de $\left|P_{e}\right\rangle$ que contribuem para o cálculo de $\chi$ são as correspondentes a vetores com 2 partículas.

- Como o operador $V$ nunca destrói partículas, apenas cria ou transfere partículas, vetores com mais de 2 partículas podem ser completamente desprezados no cálculo.

- Após o primeiro passo, os únicos sítios onde é necessário aplicar $V_{i}$ são aqueles onde existe uma única partícula no par $\{i, i+1\}$ : quando ambos os sítios estão vazios, o resultado é a criação de um vetor com 4 partículas, que é inútil para o cálculo de $\chi$, e quando os sítios estão ocupados, o operador aniquila o vetor.

Aplicando estas regras algumas vezes, vemos que é possível escrever os termos do vetor probabilidade estacionária que contribuem para o cálculo da susceptibilidade 
na forma geral:

$$
\left|P_{e}\right\rangle=\sum_{i, k=1}^{N} \sum_{l=1}^{\infty} A_{l, k}\left(\frac{\lambda}{2}\right)^{l}|i, i+k\rangle
$$

Vetores com $0,4,6, \ldots 2 l$ partículas, $l \neq 1$, são excluídos da soma. Utilizando esta forma geral para calcular a susceptibilidade (4.39), obtemos:

$$
\begin{aligned}
\chi & =1+\left\langle\phi_{0}\left|M_{h} \sum_{j(\neq h)} M_{j}\right| P_{e}\right\rangle \\
& =1+\sum_{j(\neq h)} \sum_{i, k} \sum_{l=1}^{\infty} A_{l, k}\left(\frac{\lambda}{2}\right)^{l}\langle h, j \mid i, i+k\rangle \\
& =1+\sum_{j(\neq h)} \sum_{i, k} \sum_{l=1}^{\infty} A_{l, k}\left(\frac{\lambda}{2}\right)^{l}\left[\delta_{h, i} \delta_{j, i+k}+\delta_{h, i+k} \delta_{j, i}\right] \\
& =1+\sum_{j(\neq h)} \sum_{k} \sum_{l=1}^{\infty} A_{l, k}\left(\frac{\lambda}{2}\right)^{l}\left[\delta_{j, h+k}+\delta_{j, h-k}\right] \\
& =1+2 \sum_{l=1}^{\infty} \sum_{k} A_{l, k}\left(\frac{\lambda}{2}\right)^{l}
\end{aligned}
$$

A última passagem acima é válida porque $k \neq 0$ (2 partículas não podem se sobrepor neste modelo).

De modo geral, o cálculo de uma grandeza física a partir de uma expansão em série perturbativa não inclui todos os termos produzidos na expansão, mas apenas um subconjunto desses termos. Tendo descoberto quais termos são necessários para o resultado final, os próximos passos consistem em calcular o coeficiente numérico de cada termo e transformar a série formal original numa série numérica, que pode ou não ser somável analiticamente.

\subsubsection{Cálculo dos coeficientes $A_{l, k}$}

A partir dos cálculos efetuados no item anterior, vemos que os primeiros coeficientes são: $A_{1,1}=A_{2,2}=1$, e os demais coeficientes de ordens $l=1,2$ são nulos. Nesta seção, vamos calcular uma relação de recorrência envolvendo os coeficientes $A_{l, k}$, a qual iremos resolver para obter os demais coeficientes.

Devido à simetria espacial do problema, a posição das partículas relativa à origem da cadeia não deve ser relevante, apenas a posição relativa das partículas entre si. Vamos tomar $\frac{1}{N} \sum_{i}|i, i+k\rangle=|k\rangle$ como a combinação linear de todos os vetores 
Tabela 4.1: Coeficientes $A_{l, k}$ calculados no intervalo $1 \leq l, k \leq 10$ por aplicação da relação de recorrência (4.54).

\begin{tabular}{|c|cccccccccc|}
\hline$l \backslash k$ & 1 & 2 & 3 & 4 & 5 & 6 & 7 & 8 & 9 & 10 \\
\hline 1 & 1 & 0 & 0 & 0 & 0 & 0 & 0 & 0 & 0 & 0 \\
2 & 0 & 1 & 0 & 0 & 0 & 0 & 0 & 0 & 0 & 0 \\
3 & 1 & 0 & 1 & 0 & 0 & 0 & 0 & 0 & 0 & 0 \\
4 & 0 & 2 & 0 & 1 & 0 & 0 & 0 & 0 & 0 & 0 \\
5 & 2 & 0 & 3 & 0 & 1 & 0 & 0 & 0 & 0 & 0 \\
6 & 0 & 5 & 0 & 4 & 0 & 1 & 0 & 0 & 0 & 0 \\
7 & 5 & 0 & 9 & 0 & 5 & 0 & 1 & 0 & 0 & 0 \\
8 & 0 & 14 & 0 & 14 & 0 & 6 & 0 & 1 & 0 & 0 \\
9 & 14 & 0 & 28 & 0 & 20 & 0 & 7 & 0 & 1 & 0 \\
10 & 0 & 42 & 0 & 48 & 0 & 27 & 0 & 8 & 0 & 1 \\
\hline
\end{tabular}

com duas partículas separadas pela distância $k$. Aplicando o operador $-R V$ a este vetor, obtemos:

$$
\begin{aligned}
& -R V|k\rangle=-\frac{1}{N} \sum_{i} R V|i, i+k\rangle \\
& =\frac{1}{2 N} \sum_{i}\{|i-1, i+k\rangle+|i+1, i+k\rangle+|i, i+k-1\rangle+|i, i+k+1\rangle\} \\
& =\frac{1}{N} \sum_{i}\{|i, i+k-1\rangle+|i, i+k+1\rangle\}=|k+1\rangle+|k-1\rangle
\end{aligned}
$$

supondo que $k-1 \neq 0$, uma vez que as partículas não podem se sobrepor. Como o estado $|k\rangle$ gera contribuições, na próxima ordem, proporcionais a $|k+1\rangle$ e $|k-1\rangle$, as únicas contribuições para o vetor $|k\rangle$ na ordem $l$ vem dos vetores $|k-1\rangle$ e $|k+1\rangle$ da ordem anterior. Uma exceção deve ser feita quando $k=1$, porque não definimos o vetor $|k=0\rangle$. Algebricamente:

$$
\begin{aligned}
& A_{1, k}=\delta_{k, 1} \\
& A_{l, k}=\left(1-\delta_{k, 1}\right) A_{l-1, k-1}+A_{l-1, k+1}, \quad l>1, k \geq 1
\end{aligned}
$$

A aplicação sucessiva desta relação de recorrência para $1 \leq l, k \leq 10$ produz os coeficientes da tabela 4.1 .

Obtemos uma relação de recorrência nos índices $l, k$. Um método para resolver relações de recorrência é o da função geratriz, descrito por Knuth, Patashnik e 
Grahan [30]. Nesse método, definimos a função $F\left(x_{1}, \ldots x_{d}\right)$, com um número de argumentos igual ao número de índices na relação, como a série de potências:

$$
F\left(x_{1}, \ldots x_{d}\right)=\sum_{n_{1}=0}^{\infty} \ldots \sum_{n_{d}=0}^{\infty} A_{n_{1}, \ldots n_{d}} x_{1}^{n_{1}} \ldots x_{d}^{n_{d}}
$$

Multiplicando a relação de recorrência por $x_{1}^{n_{1}} \ldots x_{d}^{n_{d}}$ e somando em todos os índices, obtemos uma equação algébrica ou diferencial para $F$. Obtendo $F$ e expandindo o resultado em série de potências dos argumentos, identificamos os coeficientes $A_{n_{1}, \ldots n_{d}}$.

Para que o método funcione de forma mais geral, é necessário incorporar na relação o conjunto de condições iniciais da relação de recorrência, o que é feito usualmente por meio de funções delta de Kronecker. Este procedimento é necessário para que se possam eliminar as restrições sobre os índices que limitam a utilidade da relação. Assim, convertemos a relação (4.54) para a forma:

$$
A_{l, k}=\left(1-\delta_{k, 1}\right) A_{l-1, k-1}+A_{l-1, k+1}+\delta_{l, 1} \delta_{k, 1}
$$

Existem alguns problemas ao tentar aplicar o método da função geratriz diretamente à relação (4.56). Em primeiro lugar, devido à presença do termo $\delta_{k, 1} A_{l-1, k-1}$, não é possível escrever uma equação envolvendo apenas a função geratriz dos coeficientes $A_{l, k}$. Outro inconveniente, de menor importância, é o fato dos $A_{l, k}$ serem diferentes de zero apenas quando $l-k$ é um número par, o que aumenta a complexidade algébrica dos cálculos.

Para resolver o segundo problema, vamos fazer a mudança de índices

$$
\{l, k\} \rightarrow\{l, m=(l-k) / 2\}
$$

Fazendo esta alteração, o coeficiente $A_{l, m}$ é não nulo no intervalo $l \geq 0,0 \leq m<$ $l / 2$. Um intervalo mais natural de valores para $m$ pode ser obtido (e os cálculos envolvendo a função geratriz tornam-se mais simples) tomando-se:

$$
\begin{aligned}
\mathcal{A}_{l, m} & =\mathcal{A}_{l, l-m}=A_{l, l-2 m}, 0 \leq m<l / 2, \\
\mathcal{A}_{l, l / 2} & =0, \quad l \geq 1, \quad l \text { par }
\end{aligned}
$$

Fixar $\mathcal{A}_{l, l / 2}=0$ corresponde a impor que as partículas não se sobreponham. 
Tabela 4.2: Coeficientes $\mathcal{A}_{l, m}$ calculados no intervalo $0 \leq l, m \leq 10$.

\begin{tabular}{|c|ccccccccccc|}
\hline$l \backslash k$ & 0 & 1 & 2 & 3 & 4 & 5 & 6 & 7 & 8 & 9 & 10 \\
\hline 0 & 1 & 0 & 0 & 0 & 0 & 0 & 0 & 0 & 0 & 0 & 0 \\
1 & 1 & 1 & 0 & 0 & 0 & 0 & 0 & 0 & 0 & 0 & 0 \\
2 & 1 & 0 & 1 & 0 & 0 & 0 & 0 & 0 & 0 & 0 & 0 \\
3 & 1 & 1 & 1 & 1 & 0 & 0 & 0 & 0 & 0 & 0 & 0 \\
4 & 1 & 2 & 0 & 2 & 1 & 0 & 0 & 0 & 0 & 0 & 0 \\
5 & 1 & 3 & 2 & 2 & 3 & 1 & 0 & 0 & 0 & 0 & 0 \\
6 & 1 & 4 & 5 & 0 & 5 & 4 & 1 & 0 & 0 & 0 & 0 \\
7 & 1 & 5 & 9 & 5 & 5 & 9 & 5 & 1 & 0 & 0 & 0 \\
8 & 1 & 6 & 14 & 14 & 0 & 14 & 14 & 6 & 1 & 0 & 0 \\
9 & 1 & 7 & 10 & 28 & 14 & 14 & 28 & 10 & 7 & 1 & 0 \\
10 & 1 & 8 & 27 & 48 & 42 & 0 & 42 & 48 & 27 & 8 & 1 \\
\hline
\end{tabular}

Com estas mudanças, a suceptibilidade passa a ser:

$$
\chi=1+2 \sum_{l=1}^{\infty} \sum_{m=0}^{l / 2}\left(\frac{\lambda}{2}\right)^{l} A_{l, m}=1+\sum_{l=1}^{\infty} \sum_{m=0}^{l}\left(\frac{\lambda}{2}\right)^{l} \mathcal{A}_{l, m}
$$

Reescrevemos agora a relação de recorrência em termos dos índices $l, m$. Notamos que há dois termos correspondentes a $l=1$ que são diferentes de zero $\left(\mathcal{A}_{1,0}=\right.$ $\left.\mathcal{A}_{1,1}=1\right)$, condição que deve ser incorporada à relação por meio de funções delta de Kronecker. Equivalentemente, podemos definir $\mathcal{A}_{0,0}=1$ e $\mathcal{A}_{0, m \neq 0}=0$, o que simplifica os cálculos. Embora o termo de ordem 0 não apareça na expressão da susceptibilidade, podemos mantê-lo durante os cálculos e, no momento de calcular $\chi$, retirar a contribuição de $l=0$. Usando essa convenção, os valores numéricos dos coeficientes $\mathcal{A}_{l, m}$, no intervalo $0 \leq l, m \leq 10$, aparecem na tabela 4.2 .

Para lidar com a presença de coeficientes multiplicados por funções delta, introduzimos uma nova sequência de coeficientes $D_{n}$, definidos como:

$$
\begin{aligned}
& D_{0}=1 \\
& D_{n}=-\left(\mathcal{A}_{2 n-1, n-1}+\mathcal{A}_{2 n-1, n}\right), n>0
\end{aligned}
$$

desacoplando os coeficientes $\mathcal{A}_{l, m}$ das funções delta. Reescrevemos a relação acima incluindo a condição inicial:

$$
D_{n}=\delta_{n, 0}-\left(\mathcal{A}_{2 n-1, n-1}+\mathcal{A}_{2 n-1, n}\right)
$$


Tabela 4.3: Coeficientes $D_{n}$, calculados no intervalo $0 \leq n \leq 5$.

\begin{tabular}{|c|cccccc|}
\hline$n$ & 0 & 1 & 2 & 3 & 4 & 5 \\
\hline$D_{n}$ & 1 & -2 & -2 & -4 & -10 & -28 \\
\hline
\end{tabular}

Os valores numéricos dos coeficientes $D_{n}, 0 \leq n \leq 5$, aparecem na tabela 4.3.

Reescrevendo (4.56) utilizando (4.58) e (4.60), obtemos a relação:

$$
\mathcal{A}_{l, m}=\mathcal{A}_{l-1, m-1}+\mathcal{A}_{l-1, m}+D_{m} \delta_{l, 2 m}
$$

O sistema de relações de recorrência (4.61) e (4.62) pode ser resolvido introduzindo-se as funções geratrizes $\mathcal{F}(x, y)$ e $G(z)$ :

$$
\begin{aligned}
\mathcal{F}(x, y) & =\sum_{l, m} \mathcal{A}_{l, m} x^{l} y^{m}, \\
G(z) & =\sum_{n} D_{n} z^{n}
\end{aligned}
$$

A seguir, multiplicamos (4.62) por $x^{l} y^{m}$ e somamos nos índices $l, m$. Obtemos:

$$
\begin{aligned}
\sum_{l=0}^{\infty} \sum_{m=0}^{\infty}\left(\mathcal{A}_{l, m}-\mathcal{A}_{l-1, m-1}-\mathcal{A}_{l-1, m}\right) x^{l} y^{m} & =\sum_{l=0}^{\infty} \sum_{m=0}^{\infty} D_{m} \delta_{l, 2 m} x^{l} y^{m} \Rightarrow \\
(1-x y-x) \sum_{l=0}^{\infty} \sum_{m=0}^{\infty} \mathcal{A}_{l, m} x^{l} y^{m} & =\sum_{m=0}^{\infty} D_{m} x^{2 m} y^{m}
\end{aligned}
$$

Introduzindo as funções geratrizes $\mathcal{F}$ e $G$ definidas acima, (4.65) assume a forma:

$$
\mathcal{F}(x, y)=\frac{G\left(x^{2} y\right)}{1-x(1+y)}
$$

Obtemos uma relação entre $\mathcal{F}(x, y)$ e $G\left(x^{2} y\right)$. Expandindo essa relação em potências de $x$ e $y$, obtemos os coeficientes $\mathcal{A}_{l, m}$ em função de $D_{n}$. A expansão em série de potências do lado direito de (4.66) é:

$$
\frac{G\left(x^{2} y\right)}{1-x(1+y)}=\sum_{r=0}^{\infty} D_{r} x^{2 r} y^{r} \sum_{p=0}^{\infty} x^{p}(1+y)^{p}=\sum_{r=0}^{\infty} D_{r} \sum_{p=0}^{\infty} x^{2 r+p} \sum_{q=0}^{p}\left(\begin{array}{l}
p \\
q
\end{array}\right) y^{r+q}
$$

Agora, vamos tomar $2 r+p=l, r+q=m$. Precisamos trocar a ordem das 
somatórias, para que as somas em $l$ e $m$ não dependam de $r$. Obtemos:

$$
\begin{aligned}
\frac{G\left(x^{2} y\right)}{1-x(1+y)} & =\sum_{l=0}^{\infty} \sum_{m=0}^{l} x^{l} y^{m} \sum_{r=0}^{\min (m, l-m)} D_{r}\left(\begin{array}{c}
l-2 r \\
m-r
\end{array}\right) \Rightarrow \\
\mathcal{A}_{l, m} & =\sum_{r=0}^{\min (m, l-m)} D_{r}\left(\begin{array}{c}
l-2 r \\
m-r
\end{array}\right)
\end{aligned}
$$

Tendo obtido $\mathcal{A}_{l, m}$ em função de $D_{r}$, substituímos em (4.61) para obter uma relação envolvendo os coeficientes $D_{n}$. Primeiramente, calculamos $\mathcal{A}_{2 n-1, n-1}$ e $\mathcal{A}_{2 n-1, n}$ :

$$
\begin{gathered}
\mathcal{A}_{2 n-1, n-1}=\sum_{r=0}^{\min (n-1,2 n-1-(n-1))} D_{r}\left(\begin{array}{c}
2 n-1-2 r \\
n-1-r
\end{array}\right)=\sum_{r=0}^{n-1} D_{r}\left(\begin{array}{c}
2(n-r)-1 \\
(n-r)-1
\end{array}\right) \\
\mathcal{A}_{2 n-1, n}=\sum_{r=0}^{\min (n, 2 n-1-(n))} D_{r}\left(\begin{array}{c}
2 n-1-2 r \\
n-r
\end{array}\right)=\sum_{r=0}^{n-1} D_{r}\left(\begin{array}{c}
2(n-r)-1 \\
n-r
\end{array}\right)
\end{gathered}
$$

Substituindo em (4.61), obtemos:

$$
\begin{aligned}
D_{n} & =\delta_{n, 0}-\sum_{r=0}^{n-1} D_{r}\left[\left(\begin{array}{c}
2(n-r)-1 \\
n-r-1
\end{array}\right)+\left(\begin{array}{c}
2(n-r)-1 \\
n-r
\end{array}\right)\right] \\
& =\delta_{n, 0}-\sum_{r=0}^{n-1} D_{r}\left(\begin{array}{c}
2(n-r) \\
n-r
\end{array}\right)
\end{aligned}
$$

onde utilizamos a identidade entre números binomiais:

$$
\left(\begin{array}{c}
l-1 \\
m-1
\end{array}\right)+\left(\begin{array}{c}
l-1 \\
m
\end{array}\right)=\left(\begin{array}{c}
l \\
m
\end{array}\right), l, m>0
$$

Uma vez que $\left(\begin{array}{l}0 \\ 0\end{array}\right)=1$, podemos reescrever (4.71) como:

$$
\sum_{r=0}^{n}\left(\begin{array}{c}
2(n-r) \\
n-r
\end{array}\right) D_{r}=\delta_{n, 0}
$$

Multiplicamos (4.73) por $z^{n}$ e somamos no índice $n$ para obter:

$$
\sum_{n=0}^{\infty} z^{n} \sum_{r=0}^{n}\left(\begin{array}{c}
2(n-r) \\
n-r
\end{array}\right) D_{r}=\sum_{n=0}^{\infty} z^{n} \delta_{n, 0}
$$


Definindo $s=n-r$, temos:

$$
\sum_{s=0}^{\infty}\left(\begin{array}{c}
2 s \\
s
\end{array}\right) z^{s} \sum_{r=0}^{\infty} D_{r} z^{r}=1
$$

No apêndice A, demonstramos que

$$
(1-4 z)^{-1 / 2}=\sum_{s=0}^{\infty}\left(\begin{array}{c}
2 s \\
s
\end{array}\right) z^{s}
$$

Usando este resultado, obtemos:

$$
\sum_{r=0}^{\infty} D_{r} z^{r}=G(z)=\sqrt{1-4 z}
$$

Os coeficientes $D_{n}$ são dados pela expansão em série de (4.77) (ver apêndice A):

$$
\begin{aligned}
& D_{0}=1 \\
& D_{n}=-\frac{1}{2 n-1}\left(\begin{array}{c}
2 n \\
n
\end{array}\right), n \geq 1
\end{aligned}
$$

Substituindo (4.78) em (4.68), obtemos os coeficientes $\mathcal{A}_{l, m}$ :

$$
\mathcal{A}_{l, m}=\left(\begin{array}{c}
l \\
m
\end{array}\right)-\sum_{n=1}^{\min (m, l-m)} \frac{1}{2 n-1}\left(\begin{array}{c}
l-2 n \\
m-n
\end{array}\right)\left(\begin{array}{c}
2 n \\
n
\end{array}\right)
$$

Para obter $A_{l, k}$, tomamos $m=(l-k) / 2$ :

$$
A_{l, k}=\left(\begin{array}{c}
l \\
\frac{l-k}{2}
\end{array}\right)-\sum_{n=1}^{(l-k) / 2} \frac{1}{2 n-1}\left(\begin{array}{c}
l-2 n \\
\frac{l-k}{2}-n
\end{array}\right)\left(\begin{array}{c}
2 n \\
n
\end{array}\right)
$$

\subsubsection{Resultados}

Substituindo (4.79) em (4.59), obtemos uma expressão em potências de $\lambda$ para a susceptibilidade:

$$
\chi=1+\sum_{l=1}^{\infty}\left(\frac{\lambda}{2}\right)^{l} \sum_{m=0}^{\infty}\left[\left(\begin{array}{c}
l \\
m
\end{array}\right)-\sum_{n=1}^{\min (m, l-m)} \frac{1}{2 n-1}\left(\begin{array}{c}
l-2 n \\
m-n
\end{array}\right)\left(\begin{array}{c}
2 n \\
n
\end{array}\right)\right]
$$


Podemos obter uma expressão fechada para $\chi$ em uma dimensão, reescrevendo (4.59) em função da função geradora $\mathcal{F}(x, y)$ :

$$
\chi=1+\left[\mathcal{F}(\lambda / 2,1)-\mathcal{A}_{0,0}\right]=\frac{G\left(\lambda^{2} / 4\right)}{1-\frac{\lambda}{2}(1+1)}=\frac{\sqrt{1-\lambda^{2}}}{1-\lambda}
$$

Vemos que $\chi$ diverge para $\lambda=1$. A divergência da susceptibilidade é uma das características principais de uma transição de fase de $2^{\mathrm{a}}$ ordem, que normalmente separa um estado desordenado de um estado ordenado. Entretanto, para o modelo de Glauber linear em uma dimensão, não existe o estado ordenado, uma vez que $\lambda$ não pode ser maior que a unidade.

Definimos o expoente crítico $\gamma$ a partir da divergência da susceptibilidade magnética no ponto crítico:

$$
\chi \cong \epsilon^{-\gamma}
$$

onde $\epsilon=\left|\lambda-\lambda_{c}\right|$. Analisando (4.82), vemos que o expoente crítico associado é $\gamma=1 / 2$. Este valor do expoente crítico é o mesmo obtido por Oliveira [19] pelo método da função de Green na cadeia linear.

\subsection{O modelo GL em $d$ dimensões}

Alguns dos resultados obtidos na seção anterior podem ser generalizados para um número qualquer $d$ de dimensões. Consideramos uma rede $d$-dimensional com dimensão linear igual a $L$, com condições periódicas de contorno. Seja r o vetor posição de um sítio na rede, ao qual associamos a variável de spin $\sigma_{\mathbf{r}}$, que pode assumir os valores \pm 1 . Para atualizar a configuração $\sigma$, escolhemos um sítio $\mathbf{r}$ e invertemos o sinal do seu spin de acordo com a taxa de transição:

$$
\omega_{\mathbf{r}}(\sigma)=\frac{1}{2}\left\{1-\frac{\lambda}{2 d} \sigma_{\mathbf{r}} \sum_{\delta} \sigma_{\mathbf{r}+\delta}\right\}
$$

onde a soma é feita sobre os primeiros vizinhos do sítio $\mathbf{r}$ e $\lambda$ é um parâmetro de controle dentro do intervalo $0 \leq \lambda \leq 1$.

Notamos que, para $d>1$, este modelo e o modelo de Glauber não são mais 
idênticos, ou seja, não existe constante $C(K)$ que satisfaça:

$$
\tanh \left(K \sum_{\delta} \sigma_{\delta}\right)=C(K) \sum_{\delta} \sigma_{\boldsymbol{\delta}}
$$

para todos os valores possíveis da soma quando $d>1$.

Queremos calcular a susceptibilidade magnética por sítio a campo nulo deste modelo, dada por:

$$
\chi=1+\left\langle\sigma_{\mathbf{r}} \sum_{\mathbf{r}^{\prime}(\neq \mathbf{r})} \sigma_{\mathbf{r}^{\prime}}\right\rangle
$$

Para fazer este cálculo, vamos trabalhar com a representação matricial deste modelo e escrever $\chi$ como uma expansão em série de potências do parâmetro $\lambda / 2 d$. Os coeficientes desta série serão obtidos ao resolvermos uma relação de recorrência que eles satisfazem, por meio da introdução de funções geratrizes. Utilizando o formalismo de funções geratrizes, vamos somar a série (4.86), relacionando $\chi$ à função geratriz dos coeficientes da série.

\subsubsection{Aplicação do método de expansão em série}

Vamos converter a equação mestra do modelo GL para a forma matricial, como fizemos na seção 4.2.1. Uma configuração $\sigma$ é representada pelo vetor $|\sigma\rangle$, e a configuração obtida ao alterar o spin do sítio $\mathbf{r}$ é $\left|\sigma^{\mathbf{r}}\right\rangle$. Definimos os operadores $T_{\mathbf{r}} \mathrm{e}$ $M_{\mathbf{r}}$ :

$$
\begin{aligned}
T_{\mathbf{r}}|\sigma\rangle & =\left|\sigma^{\mathbf{r}}\right\rangle \\
M_{\mathbf{r}}|\sigma\rangle & =\sigma_{\mathbf{r}}|\sigma\rangle
\end{aligned}
$$

O operador $W$ que satisfaz à forma matricial da equação mestra (equação 4.10) é dado por:

$$
\begin{aligned}
W & =W_{0}-\frac{\lambda}{2 d} V=\sum_{\mathbf{r}}\left\{S_{\mathbf{r}}-\frac{\lambda}{2 d} \sum_{i=1}^{d} V_{i, \mathbf{r}}\right\}, \text { com } \\
S_{\mathbf{r}} & =\frac{T_{\mathbf{r}}-I}{2} \mathrm{e} \\
V_{i, \mathbf{r}} & =\left(S_{\mathbf{r}}+S_{\mathbf{r}+\mathbf{e}_{i}}\right) M_{\mathbf{r}} M_{\mathbf{r}+\mathbf{e}_{i}}
\end{aligned}
$$

Notamos que $V_{i, \mathbf{r}}$ é um operador cuja atuação no estado $|\sigma\rangle$ depende apenas dos 
spins dos sítios $\mathbf{r}$ e $\mathbf{r}+\mathbf{e}_{i}$, onde $\mathbf{e}_{i}$ é o vetor unitário na direção $i$.

$W_{0}$ não é um operador diagonal. No processo de diagonalização, consideramos cada vetor da base original como o produto externo de vetores que representam configurações de um único sítio (4.41). Para diagonalizar $W_{0}$, diagonalizamos separadamente o operador de um sítio $S$, dado por (4.42). Os autovalores de $S$ são iguais a 0 e -1, e os autovetores à direita de $S$ são dados por (4.43).

Assim, obtemos uma nova base de vetores $|\eta\rangle=\left|\eta_{1} \ldots \eta_{L^{d}}\right\rangle=\left|\mathbf{r}_{1}, \mathbf{r}_{2}, \ldots \mathbf{r}_{N}\right\rangle$, onde $\mathbf{r}_{1}, \mathbf{r}_{2}, \ldots \mathbf{r}_{N}$ é o conjunto de sítios da rede cujas variáveis $\eta$ são iguais a 1 . Nesta representação, $W_{0}$ é diagonal:

$$
W_{0}|\eta\rangle=-\sum_{\mathbf{r}} \eta_{\mathbf{r}}|\eta\rangle=-N|\eta\rangle
$$

Aplicando os operadores $M_{\mathbf{r}}, V_{i, \mathbf{r}}$ nos vetores da nova base, obtemos

$$
\begin{aligned}
M_{\mathbf{r}}|\eta\rangle & =\left|\eta^{\mathbf{r}}\right\rangle \\
V_{i, \mathbf{r}}|\eta\rangle & =-\left(\eta_{\mathbf{r}}+\eta_{\mathbf{r}+\mathbf{e}_{i}}\right)\left|\eta^{\mathbf{r}, \mathbf{r}+\mathbf{e}_{i}}\right\rangle
\end{aligned}
$$

Aplicamos agora o método de expansão em série. O autovetor estacionário $\left|\psi_{0}\right\rangle$ é aquele onde todas as variáveis $\eta$ são iguais a 0 ("vácuo"). Aplicando $V$ ao vetor $\left|\psi_{0}\right\rangle$ :

$$
V\left|\psi_{0}\right\rangle=\sum_{i=1}^{d} \sum_{\mathbf{r}} V_{i, \mathbf{r}}\left|\psi_{0}\right\rangle=-2 \sum_{i=1}^{d} \sum_{\mathbf{r}}\left|\mathbf{r}, \mathbf{r}+\mathbf{e}_{i}\right\rangle,
$$

de modo que a componente de $1^{\mathrm{a}}$ ordem do vetor $\left|P_{e}\right\rangle$ é:

$$
\frac{\lambda}{2 d}(R V)\left|\psi_{0}\right\rangle=\frac{\lambda}{2 d} \sum_{i=1}^{d} \sum_{\mathbf{r}}\left|\mathbf{r}, \mathbf{r}+\mathbf{e}_{i}\right\rangle
$$

Calculando as componentes do vetor $\left|P_{e}\right\rangle$ até a ordem $l$, observamos que aparecem somatórios de vetores representando configurações com $2,4, \ldots, 2 l$ variáveis $\eta$ não-nulas. Tais configurações podem ser importantes para o cálculo de outras grandezas, mas para o cálculo da susceptibilidade, apenas os vetores com 2 variáveis $\eta$ não-nulas são relevantes, do mesmo modo que no caso unidimensional. Como um vetor com 4 ou mais variáveis $\eta$ não-nulas, sob a ação do operador $-R V$, produz apenas vetores com no mínimo 4 variáveis $\eta$ não-nulas, todos os vetores que possuem mais de 2 variáveis $\eta$ não-nulas podem ser desprezados para o cálculo que vamos 
fazer.

Podemos escrever o vetor probabilidade estacionária como uma combinação linear de todos os vetores $|\eta\rangle$ com 2 variáveis $\eta$ não-nulas. Devido à simetria espacial do problema, os coeficientes de cada vetor componente devem depender apenas da posição relativa entre os sítios em questão, não da sua posição em relação à origem do sistema. Assim, podemos definir os coeficientes $A_{l, \mathbf{r}}$ da expansão em série de potências de $\left|P_{e}\right\rangle$ dentro do subespaço de vetores com 2 variáveis $\eta$ não-nulas:

$$
\left|P_{e}\right\rangle=\sum_{l=1}^{\infty} \sum_{\mathbf{r}, \mathbf{r}^{\prime}}\left(\frac{\lambda}{2 d}\right)^{l} A_{l, \mathbf{r}}\left|\mathbf{r}^{\prime}, \mathbf{r}^{\prime}+\mathbf{r}\right\rangle=\frac{1}{2} \sum_{l=1}^{\infty} \sum_{\mathbf{r}}\left(\frac{\lambda}{2 d}\right)^{l} A_{l, \mathbf{r}}|\mathbf{r}\rangle
$$

Na equação acima, o fator $1 / 2$ aparece porque cada conjunto de configurações pode ser representado por dois vetores posição relativa equivalentes: $|\mathbf{r}\rangle$ e $|-\mathbf{r}\rangle$. Assim, de modo a preservar a continuidade dos índices da relação de recorrência, como veremos mais adiante, devemos definir:

$$
|\mathbf{r}\rangle=|-\mathbf{r}\rangle=\frac{1}{2} \sum_{\mathbf{r}^{\prime}}\left|\mathbf{r}^{\prime}, \mathbf{r}^{\prime}+\mathbf{r}\right\rangle
$$

A definição (4.97) vale se definimos $A_{l, 0}=0$, para todo $l \leq 1$. Notamos que não foi preciso definir $A_{0, \mathbf{r}}$.

A susceptibilidade pode ser calculada utilizando-se a forma geral de $\left|P_{e}\right\rangle$ :

$$
\begin{aligned}
\chi & =1+\left\langle\phi_{0}\left|M_{\mathbf{s}} \sum_{\mathbf{s}^{\prime} \neq \mathbf{0}} M_{\mathbf{s}+\mathbf{s}^{\prime}}\right| P_{e}\right\rangle \\
& =1+\frac{1}{2} \sum_{\mathbf{s}^{\prime} \neq \mathbf{0}} \sum_{\mathbf{r}^{\prime}} \sum_{\mathbf{r}} \sum_{l=1}^{\infty}\left(\frac{\lambda}{2 d}\right)^{l} A_{l, \mathbf{r}}\left\langle\mathbf{s}, \mathbf{s}+\mathbf{s}^{\prime} \mid \mathbf{r}^{\prime}, \mathbf{r}^{\prime}+\mathbf{r}\right\rangle \\
& =1+\frac{1}{2} \sum_{l=1}^{\infty} \sum_{\mathbf{r}}\left(\frac{\lambda}{2 d}\right)^{l} A_{l, \mathbf{r}} \sum_{\mathbf{s}^{\prime} \neq \mathbf{0}}\left[\delta_{\mathbf{s}^{\prime}, \mathbf{r}}+\delta_{\mathbf{s}^{\prime},-\mathbf{r}}\right]=1+\sum_{l=1}^{\infty} \sum_{\mathbf{r}}\left(\frac{\lambda}{2 d}\right)^{l} A_{l, \mathbf{r}}
\end{aligned}
$$

\subsubsection{Cálculo dos coeficientes $A_{l, \mathrm{r}}$}

Comparando (4.96), (4.97) e (4.98), vemos que $A_{l, \pm \mathbf{e}_{i}}=1,1 \leq i \leq d$. Podemos tomar esta igualdade como condição inicial de uma relação de recorrência envolvendo os coeficientes $A_{l, \pm \mathbf{e}_{i}}$. Para encontrar o termo geral da recorrência, vamos tomar a 
expressão do termo de ordem $l-1$ de $\left|P_{e}\right\rangle$ e calcular o termo de ordem $l$ :

$$
\begin{aligned}
& \left(\frac{\lambda}{2 d}\right)^{l}\left|P_{e}\right\rangle^{(l)}=\frac{\lambda}{2 d} R V\left|P_{e}\right\rangle^{(l-1)}=\frac{\lambda}{2 d} R \sum_{i=1}^{d} \sum_{\mathbf{r}} V_{i, \mathbf{r}} \sum_{\mathbf{r}^{\prime}, \mathbf{r}^{\prime \prime}}\left(\frac{\lambda}{2 d}\right)^{l-1} \frac{A_{l-1, \mathbf{r}^{\prime}}}{2}\left|\mathbf{r}^{\prime \prime}, \mathbf{r}^{\prime \prime}+\mathbf{r}^{\prime}\right\rangle \\
& =\left(\frac{\lambda}{2 d}\right)^{l} R \sum_{i=1}^{d} \sum_{\mathbf{r}^{\prime}, \mathbf{r}^{\prime \prime}} \frac{A_{l-1, \mathbf{r}^{\prime}}}{2}\left[V_{i, \mathbf{r}^{\prime \prime}}+V_{i, \mathbf{r}^{\prime \prime}+\mathbf{r}^{\prime}}+V_{i, \mathbf{r}^{\prime \prime}-\mathbf{e}_{i}}+V_{i, \mathbf{r}^{\prime \prime}+\mathbf{r}^{\prime}-\mathbf{e}_{i}}\right]\left|\mathbf{r}^{\prime \prime}, \mathbf{r}^{\prime \prime}+\mathbf{r}^{\prime}\right\rangle \\
& =-\left(\frac{\lambda}{2 d}\right)^{l} R \sum_{i=1}^{d} \sum_{\mathbf{r}^{\prime}, \mathbf{r}^{\prime \prime}} \frac{A_{l-1, \mathbf{r}^{\prime}}}{2}\left[\left(\left|\mathbf{r}^{\prime \prime}+\mathbf{e}_{i}, \mathbf{r}^{\prime \prime}+\mathbf{r}^{\prime}\right\rangle+\left|\mathbf{r}^{\prime \prime}, \mathbf{r}^{\prime \prime}+\mathbf{r}^{\prime}-\mathbf{e}_{i}\right\rangle\right)\left(1-\delta_{\mathbf{r}^{\prime}, \mathbf{e}_{i}}\right)\right. \\
& \left.+\left(\left|\mathbf{r}^{\prime \prime}, \mathbf{r}^{\prime \prime}+\mathbf{r}^{\prime}+\mathbf{e}_{i}\right\rangle+\left|\mathbf{r}^{\prime \prime}-\mathbf{e}_{i}, \mathbf{r}^{\prime \prime}+\mathbf{r}^{\prime}\right\rangle\right)\left(1-\delta_{\mathbf{r}^{\prime},-\mathbf{e}_{i}}\right)\right] \\
& =-2\left(\frac{\lambda}{2 d}\right)^{l} R \sum_{i=1}^{d} \sum_{\mathbf{r}^{\prime}, \mathbf{r}^{\prime \prime}} \frac{A_{l-1, \mathbf{r}^{\prime}}}{2}\left[\left(1-\delta_{\mathbf{r}^{\prime}, \mathbf{e}_{i}}\right)\left|\mathbf{r}^{\prime \prime}, \mathbf{r}^{\prime \prime}+\mathbf{r}^{\prime}-\mathbf{e}_{i}\right\rangle\right. \\
& \left.+\left(1-\delta_{\mathbf{r}^{\prime},-\mathbf{e}_{i}}\right)\left|\mathbf{r}^{\prime \prime}, \mathbf{r}^{\prime \prime}+\mathbf{r}^{\prime}+\mathbf{e}_{i}\right\rangle\right] \\
& =\left(\frac{\lambda}{2 d}\right)^{l} \sum_{i=1}^{d} \sum_{\mathbf{r}^{\prime}} \frac{A_{l-1, \mathbf{r}^{\prime}}}{2}\left[\left(1-\delta_{\mathbf{r}^{\prime}, \mathbf{e}_{i}}\right)\left|\mathbf{r}^{\prime}-\mathbf{e}_{i}\right\rangle+\left(1-\delta_{\mathbf{r}^{\prime},-\mathbf{e}_{i}}\right)\left|\mathbf{r}^{\prime}+\mathbf{e}_{i}\right\rangle\right]
\end{aligned}
$$

Tomando o produto escalar da expressão acima com o vetor $\langle\mathbf{r}|$ e dividindo por $\frac{1}{2}\left(\frac{\lambda}{2 d}\right)^{l}$, obtemos:

$$
A_{l, \mathbf{r}}=\sum_{i=1}^{d} \sum_{\mathbf{r}^{\prime}} A_{l-1, \mathbf{r}^{\prime}}\left(1-\delta_{\mathbf{r}, \mathbf{0}}\right)\left(\delta_{\mathbf{r}^{\prime}, \mathbf{r}+\mathbf{e}_{i}}+\delta_{\mathbf{r}^{\prime}, \mathbf{r}-\mathbf{e}_{i}}\right)
$$

A expressão acima é válida para $l>1$. Para que ela seja válida também para $l=1$, introduzimos a condição inicial citada anteriormente, obtendo:

$$
A_{l, \mathbf{r}}=\sum_{i=1}^{d}\left[\sum_{\mathbf{r}^{\prime}} A_{l-1, \mathbf{r}^{\prime}}\left(1-\delta_{\mathbf{r}, \mathbf{0}}\right)\left(\delta_{\mathbf{r}^{\prime}, \mathbf{r}+\mathbf{e}_{i}}+\delta_{\mathbf{r}^{\prime}, \mathbf{r}-\mathbf{e}_{i}}\right)+\delta_{l, 1}\left(\delta_{\mathbf{r}, \mathbf{e}_{i}}+\delta_{\mathbf{r},-\mathbf{e}_{i}}\right)\right]
$$

A expressão acima define $A_{l, \mathbf{r}}$ para qualquer valor $l \geq 1$. Podemos estender essa expressão para qualquer $l$, de modo a utilizar o método da função geratriz, escolhendo valores adequados para $A_{0, \mathbf{r}}$. A escolha mais natural é $A_{0, \mathbf{r}}=\delta_{\mathbf{r}, \mathbf{0}}$.

Uma dificuldade em trabalhar com a expressão (4.102) é a presença de funções delta de Kronecker $\delta_{\mathbf{r}, \mathbf{0}}$ multiplicando os coeficientes $A_{l-1, \mathbf{r}^{\prime}}$, as quais também atuam como condições iniciais desconhecidas da relação de recorrência. Tratamos desta 
ocorrência defindo novos coeficientes $D_{n}$ para desempenhar o papel de condições iniciais da relação (4.102) e escrevendo uma relação de recorrência para os $D_{n}$.

Para definir os coeficientes $D_{n}$, notamos primeiramente que os índices $l, r_{i}$ dos coeficientes obedecem a uma relação de paridade. Definindo uma função $\xi\left(A_{l, \mathbf{r}}\right)$ igual à soma dos índices do coeficiente $A_{l, \mathbf{r}}$ e calculando seu valor para todos os coeficientes não-nulos com $l=1$, temos:

$$
\xi\left(A_{l, \mathbf{r}}\right)=l+\sum_{i=1}^{n} r_{i}= \begin{cases}2, & \text { para os termos da forma } A_{1, \mathbf{e}_{i}} \\ 0, & \text { para os termos da forma } A_{1,-\mathbf{e}_{i}}\end{cases}
$$

Para os demais coeficientes, vemos da equação (4.102) que $\xi\left(A_{l, \mathbf{r}}\right)$ (a soma dos índices desse termo) possui a mesma paridade de $\xi\left(A_{l-1, \mathbf{r}^{\prime}}\left(\delta_{\mathbf{r}^{\prime}, \mathbf{r}+\mathbf{e}_{i}}+\delta_{\mathbf{r}^{\prime}, \mathbf{r}-\mathbf{e}_{i}}\right)\right)$ :

$$
\xi\left(A_{l-1, \mathbf{r}^{\prime}}\left(\delta_{\mathbf{r}^{\prime}, \mathbf{r}+\mathbf{e}_{i}}+\delta_{\mathbf{r}^{\prime}, \mathbf{r}-\mathbf{e}_{i}}\right)\right)=l-1+\sum_{i} r_{i} \pm 1=\left\{\begin{array}{l}
\xi\left(A_{l, \mathbf{r}}\right) \text { ou } \\
\xi\left(A_{l, \mathbf{r}}\right)-2
\end{array}\right.
$$

Como, para os termos iniciais da relação de recorrência, a soma dos índices é par, concluímos que a soma dos índices de todos os coeficientes pode ser escrita como:

$$
l+\sum_{i=1}^{n} r_{i}=2 n, n \geq 0
$$

Assim, os termos que multiplicam $\delta_{\mathbf{r}, \mathbf{0}}$ podem ser reescritos como:

$$
-\sum_{i=1}^{d} A_{l-1, \mathbf{r}} \delta_{\mathbf{r}, \pm \mathbf{e}_{i}}=-\sum_{i=1}^{d} A_{2 n-1, \mathbf{r}} \delta_{\mathbf{r}, \pm \mathbf{e}_{i}}=D_{n}, n \geq 1
$$

Definindo $D_{0}=1$, obtemos relações de recorrência para os coeficientes $A_{l, \mathbf{r}}$ e $D_{n}$ :

$$
\begin{aligned}
A_{l, \mathbf{r}} & =\sum_{i=1}^{d} \sum_{\mathbf{r}^{\prime}} A_{l-1, \mathbf{r}^{\prime}} \delta_{\mathbf{r}^{\prime}, \mathbf{r} \pm \mathbf{e}_{i}}+\sum_{n} D_{n} \delta_{l, 2 n} \delta_{\mathbf{r}, \mathbf{0}} \\
D_{n} & =-\sum_{i=1}^{d} A_{2 n-1, \mathbf{r}} \delta_{\mathbf{r}, \pm \mathbf{e}_{i}}+\delta_{n, 0}
\end{aligned}
$$

Para resolver o sistema de relações de recorrência (4.107) e (4.108), vamos utilizar 
o método da função geratriz. Definimos as funções:

$$
\begin{aligned}
F(\alpha, \mathbf{x}) & =\sum_{l=0}^{\infty} \alpha^{l} \prod_{i=1}^{d}\left\{\sum_{r_{i}=-\infty}^{\infty} x_{i}^{r_{i}}\right\} A_{l, \mathbf{r}} \\
G(\beta) & =\sum_{n=0}^{\infty} \beta^{n} D_{n}
\end{aligned}
$$

Multiplicamos (4.107) por $\alpha^{l} \prod_{i} x_{i}^{r_{i}}$ e somamos nos índices $l, r_{i}$, obtendo:

$$
F(\alpha, \mathbf{x})=\frac{G\left(\alpha^{2}\right)}{1-\alpha \sum_{i=1}^{d}\left(x_{i}+x_{i}^{-1}\right)}
$$

A seguir, expandimos $F(\alpha, \mathbf{x})$ em potências de $\alpha, x_{i}$. Primeiramente, expandimos o numerador e o denominador em potências de $\alpha$ :

$$
F(\alpha, \mathbf{x})=\frac{\sum_{n=0}^{\infty} \alpha^{2 n} D_{n}}{1-\alpha \sum_{i=1}^{d}\left(x_{i}+x_{i}^{-1}\right)}=\sum_{n=0}^{\infty} \alpha^{2 n} D_{n} \sum_{p=0}^{\infty} \alpha^{p}\left\{\sum_{i=1}^{d}\left(x_{i}+x_{i}^{-1}\right)\right\}^{p}
$$

Agora, expandimos $\left\{\sum_{i=1}^{d}\left(x_{i}+x_{i}^{-1}\right)\right\}^{p}$ em potências dos termos $x_{i}+x_{i}^{-1}$ usando o teorema multinomial:

$$
\begin{aligned}
& F(\alpha, \mathbf{x})=\sum_{n=0}^{\infty} \alpha^{2 n} D_{n} \sum_{p=0}^{\infty} \alpha^{p} \sum_{q_{1}=0}^{p}\left(x_{1}+x_{1}^{-1}\right)^{q_{1}}\left(\begin{array}{c}
p \\
q_{1}
\end{array}\right) \sum_{q_{2}=0}^{p-q_{1}}\left(x_{2}+x_{2}^{-1}\right)^{q_{2}}\left(\begin{array}{c}
p-q_{1} \\
q_{2}
\end{array}\right) \ldots \\
& \ldots \sum_{q_{d-1}=0}^{p-q_{1} \cdots-q_{d-2}}\left(x_{d-1}+x_{d-1}^{-1}\right)^{q_{d-1}}\left(\begin{array}{c}
p-q_{1} \cdots-q_{d-2} \\
q_{d-1}
\end{array}\right)\left(x_{d}+x_{d}^{-1}\right)^{p-q_{1} \cdots-q_{d-1}}
\end{aligned}
$$

A expressão anterior pode ser reescrita de forma mais sintética introduzindo-se $q_{d}(p)=p-\sum_{i=1}^{d-1} q_{i}$ :

$$
\begin{aligned}
F(\alpha, \mathbf{x})=\sum_{n=0}^{\infty} D_{n} & \sum_{p=0}^{\infty} \alpha^{p+2 n} \\
& \prod_{j=1}^{d-1}\left\{\sum_{q_{j}=0}^{p-\sum_{i=1}^{j-1} q_{i}}\left(x_{j}+x_{j}^{-1}\right)^{q_{j}}\left(\begin{array}{c}
p-\sum_{i=1}^{j-1} q_{i} \\
q_{j}
\end{array}\right)\right\}\left(x_{d}+x_{d}^{-1}\right)^{q_{d}}
\end{aligned}
$$

A seguir, vamos expandir, utilizando o teorema binomial, os termos $\left(x_{i}+x_{i}^{-1}\right)^{q_{i}}$ 
em função de potências das variáveis $x_{i}$. Cada termo assume a forma:

$$
\left(x_{i}+x_{i}^{-1}\right)^{q_{i}}=\sum_{t_{i}=0}^{q_{i}} x_{i}^{t_{i}}\left(x_{i}^{-1}\right)^{q_{i}-t_{i}}\left(\begin{array}{c}
q_{i} \\
t_{i}
\end{array}\right)=\sum_{t_{i}=0}^{q_{i}} x_{i}^{2 t_{i}-q_{i}}\left(\begin{array}{c}
q_{i} \\
t_{i}
\end{array}\right)=\sum_{r_{i}=-q_{i}}^{q_{i}} x_{i}^{r_{i}}\left(\begin{array}{c}
q_{i} \\
\frac{r_{i}+q_{i}}{2}
\end{array}\right)
$$

Da equação acima, notamos que $r_{i}+q_{i}$ deve ser um número par, de modo que $r_{i}$ e $q_{i}$ devem ser ou ambos pares ou ambos ímpares. Assim, o índice $r_{i}$ na última passagem deve variar de 2 em 2.

Agora, reescrevemos (4.114) usando (4.115) e identificamos o índice $l=p+2 n$, obtendo:

$$
\begin{aligned}
& F(\alpha, \mathbf{x})=\sum_{n=0}^{\infty} D_{n} \sum_{l=2 n}^{\infty} \alpha^{l} \\
& \prod_{j=1}^{d-1}\left\{\sum_{q_{j}=0}^{l-2 n-\sum_{i=1}^{j-1} q_{i}} \sum_{r_{j}=-q_{j}}^{q_{j}} x_{j}^{r_{j}}\left(\begin{array}{c}
q_{j} \\
\frac{r_{j}+q_{j}}{2}
\end{array}\right)\left(\begin{array}{c}
l-2 n-\sum_{i=1}^{j-1} q_{i} \\
q_{j}
\end{array}\right)\right\} \sum_{r_{d}=-q_{d}}^{q_{d}} x_{d}^{r_{d}}\left(\begin{array}{c}
q_{d} \\
\frac{r_{d}+q_{d}}{2}
\end{array}\right)
\end{aligned}
$$

Notamos que os somatórios nos índices $l, r_{j}$ dependem dos índices $n, q_{j}$. Para podermos escrever a forma final dos coeficientes $A_{l, \mathbf{r}}$, precisamos antes retirar estas dependências, trocando a ordem dos somatórios. Em primeiro lugar, trocamos a ordem das somas em $l$ e $n$ :

$$
\begin{aligned}
& F(\alpha, \mathbf{x})=\sum_{l=0}^{\infty} \alpha^{l} \sum_{n=0}^{\lfloor l / 2\rfloor} D_{n} \\
& \prod_{j=1}^{d-1}\left\{\sum_{q_{j}=0}^{l-2 n-\sum_{i=1}^{j-1} q_{i}} \sum_{r_{j}=-q_{j}}^{q_{j}} x_{j}^{r_{j}}\left(\begin{array}{c}
q_{j} \\
\frac{r_{j}+q_{j}}{2}
\end{array}\right)\left(\begin{array}{c}
l-2 n-\sum_{i=1}^{j-1} q_{i} \\
q_{j}
\end{array}\right)\right\} \sum_{r_{d}=-q_{d}}^{q_{d}} x_{d}^{r_{d}}\left(\begin{array}{c}
q_{d} \\
\frac{r_{d}+q_{d}}{2}
\end{array}\right)
\end{aligned}
$$

Na equação acima, $\lfloor l / 2\rfloor$ é o maior inteiro menor que $l / 2$, ou a função "piso" de $l / 2$. Agora, trocamos a ordem da soma de cada par de índices $q_{i}, r_{i}$, obtendo:

$$
\begin{aligned}
& F(\alpha, \mathbf{x})=\sum_{l=0}^{\infty} \alpha^{l} \sum_{n=0}^{\lfloor l / 2\rfloor} D_{n} \\
& \prod_{j=1}^{d}\left\{\sum_{r_{j}=-\left(l-2 n-\sum_{i=1}^{j-1} q_{i}\right)}^{l-2 n-\sum_{i=1}^{j-1} q_{i}} x_{j}^{r_{j}} \sum_{q_{j}=\left|r_{j}\right|}^{l-2 n-\sum_{i=1}^{j-1} q_{i}}\left(\begin{array}{c}
q_{j} \\
\frac{r_{j}+q_{j}}{2}
\end{array}\right)\left(\begin{array}{c}
l-2 n-\sum_{i=1}^{j-1} q_{i} \\
q_{j}
\end{array}\right)\right\}
\end{aligned}
$$


$\mathrm{Na}$ expressão acima, $q_{d}$ de fato não varia, pois é determinado pelos valores de $l$ e dos outros $q_{j}$. Entretanto, colocamos $q_{n}$ dentro da soma em $j$, porque o coeficiente binomial $\left(\begin{array}{c}l-2 n-\sum_{i=1}^{j-1} q_{i} \\ q_{j}\end{array}\right)$ reduz-se a 1 quando $j=d$. Como $r_{j}$ é fixo quando somamos em $q_{j}$, dentro da somatória devemos variar $q_{j}$ de 2 em 2 .

Devemos notar que, na expressão (4.118), a soma nos índices $r_{j}$ ainda é delimitada por todos os índices $q_{i}, i<j$. Alterando a ordem das somas de modo que as somas em todos os índices $r_{j}$ estejam à esquerda das somas em todos os índices $q_{j}$, obtemos:

$$
\begin{aligned}
F(\alpha, \mathbf{x})= & \sum_{l=0}^{\infty} \alpha^{l} \sum_{n=0}^{\lfloor l / 2\rfloor} D_{n} \prod_{j=1}^{d}\left\{\sum_{r_{j}=-\left(l-2 n-\sum_{i=1}^{j-1}\left|r_{i}\right|\right)}^{l-2 n-\sum_{i=1}^{j-1}\left|r_{i}\right|} x_{j}^{r_{j}}\right\} \\
& \prod_{k=1}^{d}\left\{\sum_{q_{k}=\left|r_{k}\right|}^{l-2 n-\sum_{i=1}^{k-1} q_{i}-\sum_{i=k+1}^{d}\left|r_{i}\right|}\left(\begin{array}{c}
q_{k} \\
\frac{r_{k}+q_{k}}{2}
\end{array}\right)\left(\begin{array}{c}
l-2 n-\sum_{i=1}^{k-1} q_{i} \\
q_{k}
\end{array}\right)\right\}
\end{aligned}
$$

Finalmente, mudamos a ordem das somas nos índices $n, r_{i}$, de modo que as somas em todos os índices $r_{i}$ estejam à esquerda da soma em $n$. Obtemos:

$$
\begin{aligned}
F(\alpha, \mathbf{x})= & \sum_{l=0}^{\infty} \alpha^{l} \prod_{j=1}^{d}\left\{\sum_{r_{j}=-\left(l-\sum_{i=1}^{j-1}\left|r_{i}\right|\right)}^{l-\sum_{i=1}^{j-1}\left|r_{i}\right|} x_{j}^{r_{j}}\right\} \sum_{n=0}^{\frac{1}{2}\left[l-\sum_{i=1}^{d}\left|r_{i}\right|\right]} D_{n} \\
& \prod_{k=1}^{d}\left\{\sum_{q_{k}=\left|r_{k}\right|}^{l-2 n-\sum_{i=1}^{k-1} q_{i}-\sum_{i=k+1}^{d}\left|r_{i}\right|}\left(\begin{array}{c}
q_{k} \\
\frac{r_{k}+q_{k}}{2}
\end{array}\right)\left(\begin{array}{c}
l-2 n-\sum_{i=1}^{k-1} q_{i} \\
q_{k}
\end{array}\right)\right\}
\end{aligned}
$$

Comparando (4.120) e (4.109), obtemos os coeficientes $A_{l, \mathbf{r}}$ em função dos coeficientes $D_{n}$ :

$$
\begin{aligned}
A_{l, \mathbf{r}}=\sum_{n=0}^{\frac{1}{2}\left[l-\sum_{i=1}^{d}\left|r_{i}\right|\right]} D_{n} \\
\prod_{k=1}^{d}\left\{\sum_{q_{k}=\left|r_{k}\right|}^{l-2 n-\sum_{i=1}^{k-1} q_{i}-\sum_{i=k+1}^{d}\left|r_{i}\right|}\left(\begin{array}{c}
q_{k} \\
\frac{r_{k}+q_{k}}{2}
\end{array}\right)\left(\begin{array}{c}
l-2 n-\sum_{i=1}^{k-1} q_{i} \\
q_{k}
\end{array}\right)\right\}
\end{aligned}
$$

Substituindo (4.121) em (4.108), obtemos uma relação de recorrência envolvendo apenas os coeficientes $D_{n}$. Vamos obter esta relação em etapas. Primeiramente, 
precisamos calcular $A_{2 n-1, \pm \mathbf{e}_{i}}$ :

$$
\begin{aligned}
& A_{2 n-1, \pm \mathbf{e}_{i}}=\sum_{m=0}^{n-1} D_{m} \prod_{j=1}^{i-1}\left\{\sum_{q_{j}=0}^{2 n-2 m-2-\sum_{k=1}^{j-1} q_{k}}\left(\begin{array}{c}
q_{j} \\
\frac{q_{j}}{2}
\end{array}\right)\left(\begin{array}{c}
2 n-2 m-1-\sum_{k=1}^{j-1} q_{k} \\
q_{j}
\end{array}\right)\right\} . \\
& \sum_{q_{i}=1}^{2 n-2 m-1-\sum_{k=1}^{i-1} q_{k}}\left(\begin{array}{c}
q_{i} \\
\frac{q_{i} \pm 1}{2}
\end{array}\right)\left(\begin{array}{c}
2 n-2 m-1-\sum_{k=1}^{j-1} q_{k} \\
q_{i}
\end{array}\right) . \\
& \prod_{j=i+1}^{d}\left\{\sum_{q_{j}=0}^{2 n-2 m-1-\sum_{k=1}^{j-1} q_{k}}\left(\begin{array}{c}
q_{j} \\
\frac{q_{j}}{2}
\end{array}\right)\left(\begin{array}{c}
2 n-2 m-1-\sum_{k=1}^{j-1} q_{k} \\
q_{j}
\end{array}\right)\right\}
\end{aligned}
$$

Na expressão acima, temos que $s_{j}=0$ para $j \neq i$. Assim, conforme discutido anteriormente, $q_{j}$ assume apenas valores pares para $j \neq i$, e valores ímpares para $j=i$.

Usando a relação entre números binomiais (4.72), podemos escrever a soma de coeficientes binomiais $A_{2 n-1, \pm \mathbf{e}_{i}}$ para um dado $i$ como:

$$
\begin{array}{r}
A_{2 n-1, \mathbf{e}_{i}}+A_{2 n-1,-\mathbf{e}_{i}}= \\
=\sum_{m=0}^{n-1} D_{m} \prod_{j=1}^{i-1}\left\{\sum_{q_{j}=0}^{2 n-2 m-2-\sum_{k=1}^{j-1} q_{k}}\left(\begin{array}{c}
q_{j} \\
\frac{q_{j}}{2}
\end{array}\right)\left(\begin{array}{c}
2 n-2 m-1-\sum_{k=1}^{j-1} q_{k} \\
q_{j}
\end{array}\right) .\right. \\
\cdot \sum_{q_{i}=1}^{2 n-2 m-1-\sum_{k=1}^{i-1} q_{k}}\left(\begin{array}{c}
q_{i}+1 \\
\frac{q_{i}+1}{2}
\end{array}\right)\left(\begin{array}{c}
2 n-2 m-1-\sum_{k=1}^{j-1} q_{k} \\
q_{i}
\end{array}\right) . \\
\cdot \prod_{j=i+1}^{d}\left\{\sum_{q_{j}=0}^{2 n-2 m-1-\sum_{k=1}^{j-1} q_{k}}\left(\begin{array}{c}
q_{j} \\
\frac{q_{j}}{2}
\end{array}\right)\left(\begin{array}{c}
2 n-2 m-1-\sum_{k=1}^{j-1} q_{k} \\
q_{j}
\end{array}\right)\right\}
\end{array}
$$

Redefinimos a variável $q_{i}$ como $q_{i}^{(\text {novo })}=q_{i}+1$. Em função da nova variável $q_{i}$, (4.123) torna-se:

$$
\begin{aligned}
A_{2 n-1, \mathbf{e}_{i}}+A_{2 n-1,-\mathbf{e}_{i}}= & \\
= & \sum_{m=0}^{n-1} D_{m} \prod_{j=1}^{i-1}\left\{\sum_{q_{j}=0}^{2 n-2 m-2-\sum_{k=1}^{j-1} q_{k}}\left(\begin{array}{c}
q_{j} \\
\frac{q_{j}}{2}
\end{array}\right)\left(\begin{array}{c}
2 n-2 m-1-\sum_{k=1}^{j-1} q_{k} \\
q_{j}
\end{array}\right)\right\} .
\end{aligned}
$$




$$
\begin{aligned}
& \sum_{q_{i}=2}^{2 n-2 m-\sum_{k=1}^{i-1} q_{k}}\left(\begin{array}{c}
q_{i} \\
\frac{q_{i}}{2}
\end{array}\right)\left(\begin{array}{c}
2 n-2 m-1-\sum_{k=1}^{j-1} q_{k} \\
q_{i}-1
\end{array}\right) . \\
& \cdot \prod_{j=i+1}^{d}\left\{\sum_{q_{j}=0}^{2 n-2 m-\sum_{k=1}^{j-1} q_{k}}\left(\begin{array}{c}
q_{j} \\
\frac{q_{j}}{2}
\end{array}\right)\left(\begin{array}{c}
2 n-2 m-1-\sum_{k=1}^{j-1} q_{k} \\
q_{j}
\end{array}\right)\right\}
\end{aligned}
$$

Nota-se que, com esta definição, todos os $q_{j}$ assumem apenas números pares. Podemos expressar este fato diretamente na expressão fazendo a substituição de índices $q_{j}=2 s_{j}$. Em particular, passa a valer a igualdade

$$
\sum_{j=1}^{d} q_{j}=2 n-2 m \Rightarrow \sum_{i=1}^{d} s_{j}=n-m
$$

A substituição a seguir irá simplificar os cálculos da função geratriz:

$$
\begin{aligned}
\left(\begin{array}{c}
2 n-2 m-1-\sum_{k=1}^{j-1} q_{k} \\
q_{i}-1
\end{array}\right) \prod_{j=1}^{i-1}\left(\begin{array}{c}
2 n-2 m-1-\sum_{k=1}^{j-1} q_{k} \\
q_{j}
\end{array}\right)= \\
\quad=\gamma_{i}\left(\begin{array}{c}
2\left(n-m-\sum_{k=1}^{j-1} s_{k}\right) \\
2 s_{i}
\end{array}\right) \prod_{j=1}^{i-1}\left(\begin{array}{c}
2\left(n-m-\sum_{k=1}^{j-1} s_{k}\right) \\
2 s_{j}
\end{array}\right)
\end{aligned}
$$

onde $\gamma_{i}$ é dado por:

$$
\gamma_{i}=\frac{2 s_{i}}{2\left(n-m-\sum_{k=1}^{j-1} s_{k}\right)} \prod_{j=1}^{i-1} \frac{2\left(n-m-\sum_{k=1}^{j-1} s_{k}-s_{j}\right)}{2\left(n-m-\sum_{k=1}^{j-1} s_{k}\right)}=\frac{s_{i}}{n-m}
$$

Substituindo (4.126) e (4.127) em (4.124), obtemos:

$$
\begin{aligned}
A_{2 n-1, \mathbf{e}_{i}}+ & A_{2 n-1,-\mathbf{e}_{i}}= \\
& =\sum_{m=0}^{n-1} D_{m} \prod_{j=1}^{d} \sum_{s_{j}=0}^{n-m-\sum_{k=1}^{j-1} s_{k}} \frac{s_{i}}{n-m}\left(\begin{array}{c}
2 s_{j} \\
s_{j}
\end{array}\right)\left(\begin{array}{c}
2\left(n-m-\sum_{k=1}^{j-1} s_{k}\right) \\
2 s_{j}
\end{array}\right)
\end{aligned}
$$

Nós podemos escrever a expressão acima, que junta os três casos $j<i, j=i$ e $j>1$, porque quando $j<i, s_{j}=n-m-\sum_{k=1}^{j-1} s_{k}$, portanto concluímos da igualdade (4.125) que $s_{i}=0$, o que anula esta parcela da soma. A parcela correspodente a $s_{j=i}=0$ também se anula, de modo que incluímos no somatório uma coleção de termos nulos. 
Podemos agora substituir (4.128) em (4.108). Obtemos:

$$
\begin{aligned}
D_{n} & =-\sum_{i=1}^{d}\left\{A_{2 n-1, \mathbf{e}_{i}}+A_{2 n-1,-\mathbf{e}_{i}}\right\}+\delta_{n, 0} \\
=- & \sum_{m=0}^{n-1} D_{m} \prod_{j=1}^{d} \sum_{s_{j}=0}^{n-m-\sum_{k=1}^{j-1} s_{k}} \sum_{i=1}^{d} \frac{s_{i}}{n-m}\left(\begin{array}{c}
2 s_{j} \\
s_{j}
\end{array}\right)\left(\begin{array}{c}
2\left(n-m-\sum_{k=1}^{j-1} s_{k}\right) \\
2 s_{j}
\end{array}\right)+\delta_{n, 0} \\
& =-\sum_{m=0}^{n-1} D_{m} \prod_{j=1}^{d} \sum_{s_{j}=0}^{n-m-\sum_{k=1}^{j-1} s_{k}}\left(\begin{array}{c}
2 s_{j} \\
s_{j}
\end{array}\right)\left(\begin{array}{c}
2\left(n-m-\sum_{k=1}^{j-1} s_{k}\right) \\
2 s_{j}
\end{array}\right)+\delta_{n, 0}
\end{aligned}
$$

Notamos que, no lado direito da expressão acima, se tomarmos $n=m$, todos os índices $s_{j}$ devem ser nulos, e os números binomiais são iguais a 1. Assim, podemos escrever (4.129) como:

$$
\sum_{m=0}^{n} D_{m} \prod_{j=1}^{d} \sum_{s_{j}=0}^{n-m-\sum_{k=1}^{j-1} s_{k}}\left(\begin{array}{c}
2 s_{j} \\
s_{j}
\end{array}\right)\left(\begin{array}{c}
2\left(n-m-\sum_{k=1}^{j-1} s_{k}\right) \\
2 s_{j}
\end{array}\right)=\delta_{n, 0}
$$

Esta relação de recorrência para os $D_{m}$ pode ser resolvida pelo método da função geratriz. Multiplicando (4.130) por $z^{n}$ e somando em $n$, obtemos:

$$
\sum_{n=0}^{\infty} z^{n} \sum_{m=0}^{n} D_{m} \prod_{j=1}^{d} \sum_{s_{j}=0}^{n-m-\sum_{k=1}^{j-1} s_{k}}\left(\begin{array}{c}
2 s_{j} \\
s_{j}
\end{array}\right)\left(\begin{array}{c}
2\left(n-m-\sum_{k=1}^{j-1} s_{k}\right) \\
2 s_{j}
\end{array}\right)=\sum_{n=0}^{\infty} z^{n} \delta_{n, 0}=1
$$

A seguir, introduzimos a variável $h=n-m$ :

$$
\sum_{m=0}^{\infty} D_{m} z^{m} \sum_{h=0}^{\infty} \prod_{j=1}^{d} \sum_{s_{j}=0}^{h-\sum_{k=1}^{j-1} s_{k}}\left(\begin{array}{c}
2 s_{j} \\
s_{j}
\end{array}\right)\left(\begin{array}{c}
2\left(h-\sum_{k=1}^{j-1} s_{k}\right) \\
2 s_{j}
\end{array}\right) z^{h}=1
$$

E utilizamos a definição da função geratriz $G(z)$ :

$$
\begin{aligned}
& G(z)=\sum_{m=0}^{\infty} D_{m} z^{m}= \\
&=\left\{\sum_{h=0}^{\infty} \prod_{j=1}^{d} \sum_{s_{j}=0}^{h-\sum_{k=1}^{j-1} s_{k}}\left(\begin{array}{c}
2 s_{j} \\
s_{j}
\end{array}\right)\left(\begin{array}{c}
2\left(h-\sum_{k=1}^{j-1} s_{k}\right) \\
2 s_{j}
\end{array}\right) z^{h}\right\}^{-1}=\frac{1}{H(z)}
\end{aligned}
$$


Se encontrarmos uma expressão analítica para a função $H(z)$ definida acima em alguma dimensão $d$, será possível expandir a função $1 / H(z)$ para obter uma expressão explícita para os coeficientes $D_{n}$ e, substituindo esta expressão em (4.121), uma expressão para os coeficientes $A_{l, \mathbf{r}}$. Entretanto, não foi possível encontrar uma expressão analítica para $H(z)$ para qualquer $d$. O resultado para $d=1$ foi mostrado na seção 4.2 , e para $d=2$, discutimos uma identidade envolvendo $H(z)$ na seção seguinte.

\subsubsection{Resultados}

A susceptibilidade magnética a campo nulo para o modelo de Glauber linear pode ser calculada mesmo sem o conhecimento de uma expressão analítica para os coeficientes $A_{l, \mathbf{r}}$. De fato, comparando as equações (4.99) e (4.109), obtemos:

$$
\chi=1+\sum_{l=1}^{\infty} \sum_{\mathbf{r}}\left(\frac{\lambda}{2 d}\right)^{l} A_{l, \mathbf{r}}=\sum_{l=0}^{\infty} \sum_{\mathbf{r}}\left(\frac{\lambda}{2 d}\right)^{l} A_{l, \mathbf{r}}=F\left(\frac{\lambda}{2 d}, \mathbf{e}\right)
$$

onde e é o vetor cujas componentes são todas iguais a 1.

Da relação (4.111) entre as funções geratrizes, obtemos:

$$
\chi=\frac{G\left(\lambda^{2} / 4 d^{2}\right)}{1-\frac{\lambda}{2 d} \sum_{i=1}^{d}\left(1+1^{-1}\right)}=\frac{G\left(\lambda^{2} / 4 d^{2}\right)}{1-\lambda}
$$

e, substituindo (4.133) na equação acima, obtemos:

$$
\chi=\frac{1}{(1-\lambda) H\left(\lambda^{2} / 4 d^{2}\right)}
$$

O modelo de Glauber linear foi discutido por Oliveira [19], em um número qualquer de dimensões, com o auxílio da técnica de funções de Green de rede. Foi obtido o resultado:

$$
\chi=\frac{\lambda}{(1-\lambda) G_{0}((1-\lambda) / \lambda)}
$$

onde $G_{\mathbf{0}}(s)$ corresponde ao valor da função de Green $G_{\mathbf{r}}(s)$ na origem da rede hipercúbica de dimensão $d$ :

$$
G_{\mathbf{r}}(s)=\int_{B z} \frac{d^{d} k}{(2 \pi)^{d}} \frac{e^{i \mathbf{k} . \mathbf{r}}}{s+\frac{1}{d} \sum_{j=1}^{d}\left(1-\cos k_{j}\right)}
$$


É possível mostrar a equivalência dos resultados (4.136) e (4.137), para qualquer dimensão $d$. Em primeiro lugar, vamos utilizar (4.138) para calcular $G_{\mathbf{0}}((1-\lambda) / \lambda)$ :

$$
\begin{aligned}
G_{0}\left(\frac{1-\lambda}{\lambda}\right) & =\int_{B z} \frac{d^{d} k}{(2 \pi)^{d}} \frac{1}{\left(\frac{1-\lambda}{\lambda}\right)+1-\frac{1}{d} \sum_{j=1}^{d} \cos k_{j}} \\
& =\lambda \int_{B z} \frac{d^{d} k}{(2 \pi)^{d}} \frac{1}{1-\frac{\lambda}{d} \sum_{j=1}^{d} \cos k_{j}}
\end{aligned}
$$

Substituindo este resultado em (4.137), obtemos:

$$
\chi=\left[(1-\lambda) \int_{B z} \frac{d^{d} k}{(2 \pi)^{d}} \frac{1}{1-\frac{\lambda}{d} \sum_{j=1}^{d} \cos k_{j}}\right]^{-1}
$$

Estamos trabalhando com o intervalo $0 \leq \lambda<1$. Vamos expandir o integrando de (4.140) em série de potências de $\lambda$ e, no intervalo onde esta série e (4.136) convergirem, vamos mostrar que as expansões são idênticas. Obtemos:

$$
\begin{aligned}
& \frac{1}{1-\frac{\lambda}{d} \sum_{j=1}^{d} \cos k_{j}}=\sum_{l=0}^{\infty}\left(\frac{\lambda}{d} \sum_{j=1}^{d} \cos k_{j}\right)^{l}= \\
& =\sum_{l=0}^{\infty}\left(\frac{\lambda}{d}\right)^{l} \prod_{j=1}^{l-1}\left[\sum_{m_{j}=0}^{l-\sum_{i=1}^{j-1} m_{i}}\left(\begin{array}{c}
l-\sum_{i=1}^{j-1} m_{i} \\
m_{j}
\end{array}\right) \cos ^{m_{j}} k_{j}\right] \cos ^{m_{d}} k_{d}
\end{aligned}
$$

onde $m_{d}=l-\sum_{i=1}^{d-1} m_{i}$

Para continuarmos, podemos obter a integral de $\cos ^{m_{j}} k_{j}$ consultando Gradshteyn e Ryzhik [31]. Para $m_{j}$ ímpar, a integral se anula, enquanto para $m_{j}$ par, a integral é:

$$
\int_{0}^{2 \pi} \frac{d k}{2 \pi} \cos ^{2 s} k=\frac{(2 s-1) ! !}{(2 s) ! !}=\frac{1}{2^{2 s}}\left(\begin{array}{c}
2 s \\
s
\end{array}\right)
$$

Como o resultado (4.141) só não se anula quando todos os $m_{j}$ são pares, vamos fazer as substituições:

$$
\begin{aligned}
m_{j} & =2 s_{j} \\
l & =\sum_{j=1}^{d} m_{j}=2 \sum_{j=1}^{d} s_{j}=2 h
\end{aligned}
$$


Agora podemos escrever:

$$
\begin{aligned}
& \chi=\left\{(1-\lambda) \int_{B z} \frac{d_{d} k}{(2 \pi)^{d}} \sum_{h=0}^{\infty}\left(\frac{\lambda}{d}\right)^{2 h}\right. \\
&\left.\prod_{j=1}^{d-1}\left[\sum_{s_{j}=0}^{h-\sum_{i=1}^{j-1} s_{i}}\left(\begin{array}{c}
2\left(h-\sum_{i=1}^{j-1} s_{i}\right) \\
2 s_{j}
\end{array}\right) \cos ^{2 s_{j}} k_{j}\right] \cos ^{2 s_{d}} k_{d}\right\}^{-1} \\
&=\left\{(1-\lambda) \sum_{h=0}^{\infty}\left(\frac{\lambda}{d}\right)^{2 h} \prod_{j=1}^{d} \sum_{s_{j}=0}^{h-\sum_{i=1}^{j-1} s_{i}}\left(\begin{array}{c}
2\left(h-\sum_{i=1}^{j-1} s_{i}\right) \\
2 s_{j}
\end{array}\right) \int_{0}^{2 \pi} \frac{d k_{j}}{2 \pi} \cos ^{2 s_{j}} k_{j}\right\}^{-1} \\
&=\left\{(1-\lambda) \sum_{h=0}^{\infty}\left(\frac{\lambda}{2 d}\right)^{2 h} \prod_{j=1}^{d} \sum_{s_{j}=0}^{h-\sum_{i=1}^{j-1} s_{i}}\left(\begin{array}{c}
2\left(h-\sum_{i=1}^{j-1} s_{i}\right) \\
2 s_{j}
\end{array}\right)\left(\begin{array}{c}
2 s_{j} \\
s_{j}
\end{array}\right)\right\}^{-1} \\
&=\left\{(1-\lambda) H\left(\frac{\lambda^{2}}{4 d^{2}}\right)\right\}^{-1}
\end{aligned}
$$

onde $H(z)$ é a função definida em (4.133). Notamos que (4.136) e (4.145) coincidem em todos os pontos onde as expansões em série convergem.

Para $d=2$, a equivalência entre (4.136) e (4.145) também pode ser demonstrada reescrevendo a função $H(z)$ e a função de Green na rede em termos de integrais elípticas (ver Arfken[33] e Gradshteyn e Ryzhik[31]). Vamos mostrar essa equivalência em duas partes. Primeiramente, vamos reescrever $H(z)$, efetuando uma das somas explicitamente. Da equação (4.133) em duas dimensões, temos:

$$
H(z)=\sum_{h=0}^{\infty} z^{h} \sum_{s=0}^{h}\left(\begin{array}{c}
2 h \\
2 s
\end{array}\right)\left(\begin{array}{c}
2 s \\
s
\end{array}\right)\left(\begin{array}{c}
2(h-s) \\
h-s
\end{array}\right)=\sum_{h=0}^{\infty} z^{h} \sum_{s=0}^{h} \frac{(2 h) !}{(s !)^{2}[(h-s) !]^{2}}
$$

É possível demonstrar (apêndice B), com o algoritmo de Gosper-Zeilberger para somas de termos hipergeométricos (tais como números binomiais), a identidade:

$$
\sum_{s=0}^{h} \frac{(2 h) !}{(s !)^{2}[(h-s) !]^{2}}=\left(\begin{array}{c}
2 h \\
h
\end{array}\right)^{2}
$$

de modo que $H(z)$ é dado pela soma:

$$
H(z)=\sum_{h=0}^{\infty}\left(\begin{array}{c}
2 h \\
h
\end{array}\right)^{2} z^{h}=1+\sum_{h=1}^{\infty}\left[\frac{(2 h-1) ! !}{(2 h) ! !}\right]^{2}(16 z)^{h}
$$


Para a segunda parte da demonstração, começamos reescrevendo (4.139):

$$
\begin{aligned}
G_{\mathbf{0}}\left(\frac{1-\lambda}{\lambda}\right) & =\lambda \iint_{B z} \frac{d k_{1} d k_{2}}{(2 \pi)^{2}} \frac{1}{\left(1-\frac{\lambda}{2}\left(\cos k_{1}+\cos k_{2}\right)\right.} \\
& =2 \iint_{B z} \frac{d k_{1} d k_{2}}{(2 \pi)^{2}} \frac{1}{\frac{2}{\lambda}-\cos k_{1}-\cos k_{2}}
\end{aligned}
$$

Agora, relacionamos (4.149) com uma identidade demonstrada por Morita e Horiguchi [32], que mostraram que é possível escrever a função de Green para a rede retangular (que inclui a rede quadrada como caso especial) utilizando integrais elípticas completas ${ }^{1}$ :

$$
\mathcal{G}_{0}(s, \Gamma)=\iint_{B z} \frac{d k_{1} d k_{2}}{(2 \pi)^{2}} \frac{1}{s-\Gamma \cos x-\cos y}=\frac{k_{1} K\left(k_{1}^{2}\right)}{\pi \sqrt{\gamma}},
$$

onde $\Gamma$ é a razão entre as arestas da célula unitária da rede,

$$
k_{1}=\left(\frac{4 \Gamma}{s^{2}-(\Gamma-1)^{2}}\right)^{1 / 2}
$$

e $K(m)$ é a integral elíptica completa do $1^{\circ}$ tipo (complete elliptic integral of the fisrt kind), dada por (definição de Arfken [33]):

$$
K(m)=\int_{0}^{\pi / 2} \frac{d \theta}{\sqrt{1-m \sin ^{2} \theta}}
$$

Para a rede quadrada, $\Gamma=1$, de modo que $k_{1}=2 / s$. Substituindo na expressão (4.150), obtemos:

$$
\mathcal{G}_{0}(s, 1)=\frac{2}{\pi s} K\left\{\left(\frac{2}{s}\right)^{2}\right\}
$$

Comparando (4.149) e (4.150), é fácil ver que:

$$
G_{0}\left(\frac{1-\lambda}{\lambda}\right)=2 \mathcal{G}_{0}\left(\frac{2}{\lambda}, 1\right) \stackrel{(4.153)}{=} \frac{2 \lambda}{\pi} K\left(\lambda^{2}\right),
$$

\footnotetext{
${ }^{1}$ Vemos que Oliveira [19] e Morita e Horiguchi [32] utilizam definições ligeiramente diferentes para a função de Green de rede, de modo que utlizamos $G$ para a primeira definição e $\mathcal{G}$ para a segunda. Além disso, Morita e Horiguchi utilizam uma definição diferente para a integral elíptica $K$ da utilizada por Arfken[33], de modo que a equação que aparece no trbablho de Morita e Horiguchi relaciona $\mathcal{G}_{0}(s, \Gamma)$ e $K\left(k_{1}\right)$. Aqui, nós corrigimos essa equação de acordo com a definição utilizada por Arfken.
} 
de modo que a expressão de Oliveira para a susceptibilidade do modelo GL em duas dimensões pode ser reescrita da seguinte forma:

$$
\chi_{d=2}=\frac{\lambda}{1-\lambda}\left\{\frac{2 \lambda}{\pi} K\left(\lambda^{2}\right)\right\}^{-1}=\frac{\pi}{2(1-\lambda) K\left(\lambda^{2}\right)}
$$

Completamos a prova da equivalência entre os resultados (4.136) e (4.137) expandindo $K(m)$ em série de potências. Consultando Arfken [33], vemos que $K(m)$ possui a seguinte expansão em potências do parâmetro $m$ :

$$
K(m)=\frac{\pi}{2}\left\{1+\sum_{n=1}^{\infty}\left[\frac{(2 n-1) ! !}{(2 n) ! !}\right]^{2} m^{n}\right\}
$$

Comparando as expansões (4.148) e (4.156), vemos que valem as identidades:

$$
H(z)=\frac{2 K(16 z)}{\pi} \Rightarrow \frac{1}{(1-\lambda) H\left(\lambda^{2} / 16\right)}=\frac{\pi}{2(1-\lambda) K\left(\lambda^{2}\right)}
$$

Oliveira também calculou o expoente crítico $\gamma$, associado à divergência da susceptibilidade em $\lambda=1$, mostrando que $G_{\mathbf{0}}(0)$ é finita quando $d>2$, divergindo de forma logarítmica quando $d=2$ e de forma exponencial, com o expoente $d / 2-1$, para $d<2$ [19]. Deste modo,

$$
\gamma= \begin{cases}d / 2, & \text { para } d<2, \text { e } \\ 1, & \text { para } d \geq 2, \text { com correções logarítmicas para } d=2\end{cases}
$$

\subsection{Expansão em série para o modelo de Glauber em 2 dimensões}

Nas seções anteriores, discutimos como calcular grandezas físicas para o modelo de Glauber linear pelo procedimento de expansão em série perturbativa. Como discutido na seção 4.1, embora nos dois casos tenhamos encontrado resultados analíticos importantes, que podem ser colocados numa forma fechada ou relacionados com alguma integral conhecida, na grande maioria dos casos não é possível reduzir a série produzida pelo processo a alguma forma analítica. Nesses casos, deve-se optar por uma análise numérica da série, obtendo-se o maior número de termos $\left|P_{l}\right\rangle$ possível e estimando-se o erro cometido ao desprezar os demais termos. Nas vizinhanças 
de um ponto crítico, onde todos os termos devem se tornar importantes para a descrição física do modelo, ainda é possível fazer uma análise de Padé [23] dos resultados, de modo a estimar expoentes críticos. Nesta seção, vamos discutir como obter uma grandeza física a partir de uma série produzida numericamente, tomando como exemplo o cálculo da autocorrelação temporal do modelo de Glauber em duas dimensões.

Em duas dimensões, como comentado anteriormente, o estado estacionário do modelo de Glauber apresenta uma transição de fase entre um estado ferromagnético (ordenado) e um estado paramagnético (desordenado) a uma temperatura crítica $T_{c}$ tal que $\sinh 2 K=\sinh J / k_{B} T_{c}=1$. A correlação temporal entre os spins $\sigma_{\mathbf{0}}$ e $\sigma_{\mathbf{r}}$ para o modelo de Glauber pode ser calculada como:

$$
C_{\mathbf{r}}(t)=\sum_{\sigma, \sigma^{\prime}} \sigma_{\mathbf{r}}^{\prime} W\left(\sigma^{\prime}, \sigma ; t\right) \sigma_{0} P(\sigma, 0)
$$

onde $W\left(\sigma^{\prime}, \sigma ; t\right)$ é a probabilidade de transição, num intervalo de tempo $t$, entre a configuração inicial $\sigma$ e a configuração final $\sigma^{\prime}$, podendo passar por configurações intermediárias. Tomando $\mathbf{r}=\mathbf{0}$, obtemos a autocorrelação temporal $C_{\mathbf{0}}(t)$,

Fora do ponto crítico, $C_{\mathbf{0}}(t)$ deve decair exponencialmente. Entretanto, no ponto crítico $\left(T=T_{c}\right), C_{\mathbf{0}}(t)$ deve apresentar um decaimento algébrico:

$$
C_{\mathbf{0}}(t)= \begin{cases}A e^{-t / \tau}, & \text { fora do ponto crítico } \\ A_{c} t^{-\nu_{\|}}, & \text {no ponto crítico }\end{cases}
$$

Assim, o exponente crítico $\nu_{\|}$, que descreve o comportamento de correlações temporais no ponto crítico, pode ser calculado analisando o comportamento da função $C_{\mathbf{0}}(t)$ no ponto crítico.

É possível calcular $C_{\mathbf{0}}(t)$ pelo método de expansão em série utilizado nos capítulos anteriores. Primeiramente, convertemos (4.159) para a forma matricial com $\mathbf{r}=\mathbf{0}$, obtendo:

$$
\begin{aligned}
C_{\mathbf{0}}(t) & =\left\langle\phi_{0}\left|M_{\mathbf{0}} \exp (t W) M_{\mathbf{0}}\right| \psi_{0}\right\rangle \\
& =\left\langle\phi_{0}\left|M_{\mathbf{0}}\left\{I+t W+\frac{t^{2} W^{2}}{2 !}+\ldots\right\} M_{\mathbf{0}}\right| \psi_{0}\right\rangle
\end{aligned}
$$

uma vez que $\exp (t W)$ é o operador de evolução temporal ligando os instantes 0 e $t$. Para escrever este operador na forma matricial precisamos converter a função tanh, 
que aparece na taxa de transição do modelo de Glauber:

$$
w_{\mathbf{r}}(\sigma)=\frac{1}{2}\left\{1-\sigma_{\mathbf{r}} \tanh \left(K \sum_{\delta} \sigma_{\mathbf{r}+\boldsymbol{\delta}}\right)\right\},
$$

em operadores matriciais.

O procedimento padrão para fazer essa mudança é escrever a função $\tanh (x)$ como uma série infinita de potências de seu argumento. Uma vez que essa função é ímpar, apenas potências ímpares do argumento são esperadas na sua expansão em série. Em particular, os primeiros termos desa expansão são:

$$
\tanh x=x-\frac{x^{3}}{3}-\frac{11 x^{5}}{30}+O\left(x^{7}\right)
$$

Concluímos que apenas combinações lineares de produtos de um número ímpar de operadores $M_{\mathbf{r}+\boldsymbol{\delta}}$ devem aparecer na representação matricial da equação mestra.

Entretanto, não é necessário considerar um número infinito de produtos de operadores na representação matricial da equação mestra. Uma vez que a soma das variáveis $\sigma_{\mathbf{r}+\boldsymbol{\delta}}$ no argumento da função tangente hiperbólica assume um número finito de valores ( $2 d+1$ valores para uma rede hipercúbica em $d$ dimensões), podemos substituir a função tanh em (4.162) por uma combinação linear de produtos de no máximo $n$ variáveis de spin $\sigma_{\mathbf{r}+\boldsymbol{\delta}}$, onde $n$ depende de $d$. Para $d=1, n=1$ e obtemos o modelo de Glauber linear.

Em duas dimensões, verificamos que $n=3$ : a taxa de transição do modelo de Glauber (4.162) é equivalente à taxa do modelo de Glauber linear, acrescida de um termo proporcional ao produto de três variáveis de spin dos sítios vizinhos:

$$
w_{\mathbf{r}}(\sigma)=\frac{1}{2}\left\{1-\frac{\sigma_{\mathbf{r}}}{4} \sum_{\boldsymbol{\delta}} \sigma_{\mathbf{r}+\boldsymbol{\delta}}\left(A+B \prod_{\boldsymbol{\delta}^{\prime}} \sigma_{\mathbf{r}+\boldsymbol{\delta}^{\prime}}\right)\right\}
$$

Na expressão acima, como $\sigma_{\mathbf{r}}^{2}=1$, o produto de cinco spins vizinhos que multiplica $B$ na verdade depende apenas de três spins vizinhos.

Para obter a relação entre as taxas de transição (4.162) e (4.164), impomos que a igualdade a seguir seja satisfeita para todos os possíveis valores do argumento:

$$
\tanh \left(K \sum_{\boldsymbol{\delta}} \sigma_{\mathbf{r}+\boldsymbol{\delta}}\right)=\sum_{\boldsymbol{\delta}} \sigma_{\mathbf{r}+\boldsymbol{\delta}}\left(A+B \prod_{\boldsymbol{\delta}^{\prime}} \sigma_{\mathbf{r}+\boldsymbol{\delta}^{\prime}}\right)
$$




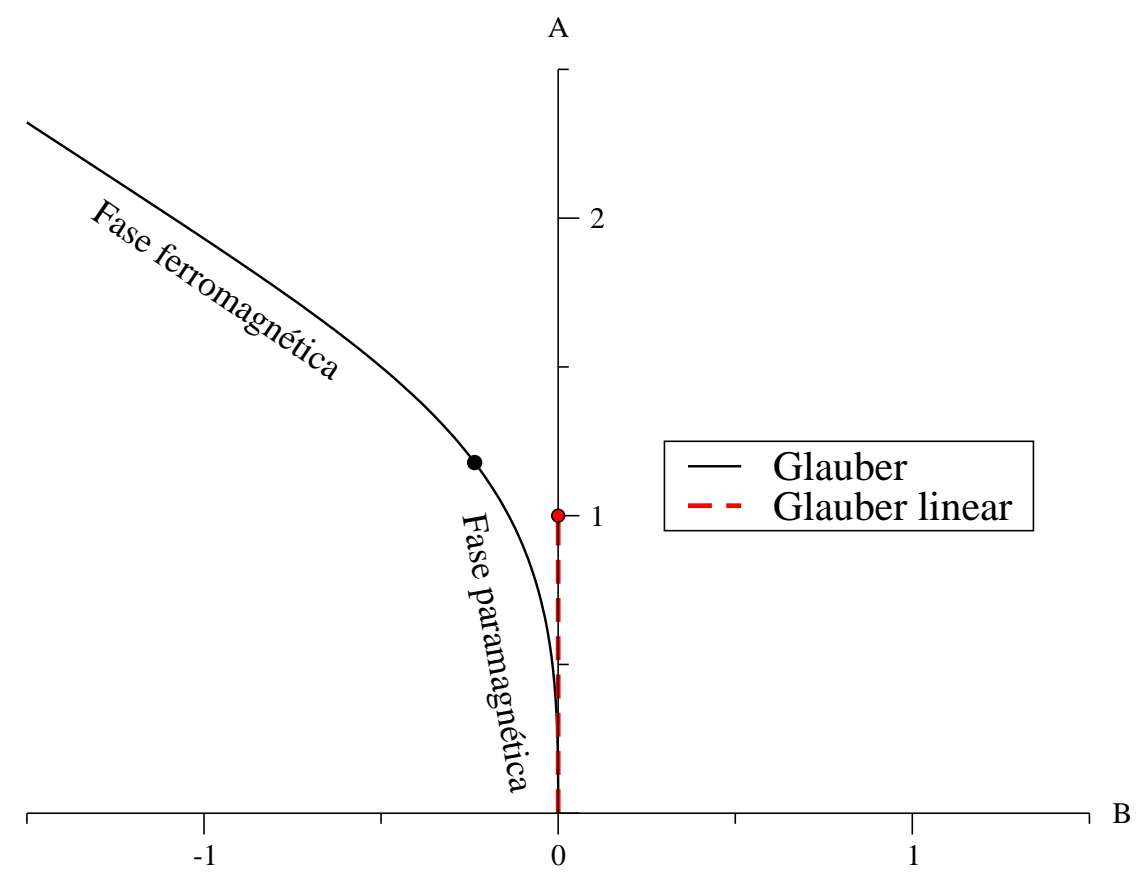

Figura 4.2: Variação dos parâmetros $A$ (linear) e $B$ (cúbico) para os modelos de Glauber e Glauber linear. Os círculos marcam os pontos críticos do modelo de Glauber $(A=5 / 3 \sqrt{2}, B=-1 / 3 \sqrt{2})$ e do modelo de Glauber linear $(A=1, B=0)$, que separam uma fase ordenada, acima do ponto crítico, de uma fase desordenada, abaixo desse ponto.

Tomando $\mu=\tanh 2 K$, obtemos os valores a seguir para as constantes $A$ e $B$ :

$$
A=\frac{\mu\left(2+\mu^{2}\right)}{1+\mu^{2}}, \quad B=-\frac{\mu^{3}}{1+\mu^{2}}
$$

Variando o parâmetro $\mu$ no intervalo $0 \leq \mu<\infty$, obtemos o modelo de Glauber em equilíbrio com um banho térmico a qualquer temperatura.

O espaço total de variação dos parâmetros $A$ e $B$ define um modelo mais geral, o qual batizamos modelo de Glauber generalizado (modelo GG). Este modelo inclui como casos específico o modelo de Glauber, quando existe $\mu$ tal que (4.166) seja satisfeita, e o modelo de Glauber linear, quando $A=\lambda$ e $B=0$ (ver figura 4.2). Assim, se conseguirmos obter o expoente crítico $\nu_{\|}$para o modelo de Glauber em duas dimensões por este método, também será possível estudar o modelo GG para quaisquer valores de $A$ e $B$, procurando pontos críticos. 


\subsubsection{Expansão em série perturbativa}

Tendo convertido a taxa de transição do modelo de Glauber em um produto de variáveis $\sigma_{\mathbf{r}}$, podemos construir a matriz de evolução $W$ para o modelo de Glauber. Seja $\mathbf{r}=\left\{r_{1}, r_{2}\right\}$ o vetor posição de um sítio na rede. Definimos os vetores de estado como no capítulo anterior, e os operadores $T_{\mathbf{r}}, M_{\mathbf{r}}$ e $S_{\mathbf{r}}$ como anteriormente.

O operador $W$ é dado por:

$$
\begin{aligned}
W & =W_{0}-\frac{1}{4}\left(A V^{(2)}+B V^{(4)}\right), \text { onde } \\
W_{0} & =\sum_{\mathbf{r}} S_{\mathbf{r}}, \\
V^{(2)} & =\sum_{\mathbf{r}} \sum_{\boldsymbol{\delta}} V_{\mathbf{r}, \boldsymbol{\delta}}^{(2)}=\sum_{\mathbf{r}} \sum_{\boldsymbol{\delta}} S_{\mathbf{r}} M_{\mathbf{r}} M_{\mathbf{r}+\boldsymbol{\delta}}, \\
V^{(4)} & =\sum_{\mathbf{r}} \sum_{\boldsymbol{\delta}} V_{\mathbf{r}, \boldsymbol{\delta}}^{(4)}=\sum_{\mathbf{r}} \sum_{\boldsymbol{\delta}} S_{\mathbf{r}} M_{\mathbf{r}} M_{\mathbf{r}+\boldsymbol{\delta}} \prod_{\boldsymbol{\delta}^{\prime}} M_{\mathbf{r}+\boldsymbol{\delta}^{\prime}}
\end{aligned}
$$

e $\boldsymbol{\delta}, \boldsymbol{\delta}^{\prime}$ são um dos quatro vetores unitários da rede.

Diagonalizamos $W_{0}$ mudando para a base de vetores $|\eta\rangle=\left|\eta_{1}, \ldots \eta_{i} \ldots \eta_{L^{2}}\right\rangle$. Os autovalores de $W_{0}$ são da forma $\Lambda_{k}=-N$, onde $N$ é o número de variáveis $\eta$ iguais a 1. A ação dos operadores $S_{\mathbf{r}}, V_{\mathbf{r}, \boldsymbol{\delta}}^{(2)}$ e $V_{\mathbf{r}, \boldsymbol{\delta}}^{(4)}$ sobre um vetor $|\eta\rangle$ aparece no diagrama 4.3 .

Na forma matricial, a função de autocorrelação temporal é dada por:

$$
C_{\mathbf{0}}(t)=\left\langle 1_{\mathbf{0}}|\exp (t W)| 1_{\mathbf{0}}\right\rangle
$$

onde $\left\langle 1_{\mathbf{0}}\right|$ e $\left|1_{\mathbf{0}}\right\rangle$ são o vetor linha e o vetor coluna obtidos aplicando-se o operador $M_{\mathbf{0}}$ ao vetor linha $\left\langle\phi_{\mathbf{0}}\right|$ e ao vetor coluna $\left|\psi_{\mathbf{0}}\right\rangle$. O índice $\mathbf{0}$ significa que ambos os operadores devem atuar no mesmo sítio da rede, o qual podemos tomar como a origem $\mathbf{0}=(0,0)$. Podemos ainda escrever, utilizando a expansão formal do operador $\exp (t W)$ :

$$
\begin{aligned}
C_{\mathbf{0}}(t) & =\sum_{l} c_{l} t^{l}, \text { com } \\
c_{l} & =\frac{1}{l !}\left\langle 1_{\mathbf{0}}\left|\left(W_{0}-\frac{A}{4} V^{(2)}-\frac{B}{4} V^{(4)}\right)^{l}\right| 1_{\mathbf{0}}\right\rangle
\end{aligned}
$$

A seguir, vamos utilizar a representação em variáveis $\eta$ como um modelo de gás de rede para discutir o processo de criação dos termos da expansão em série, 

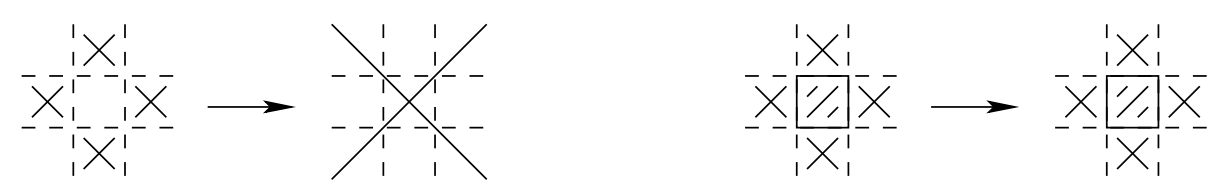

(a) Ação do operador $S_{\mathrm{r}}$.
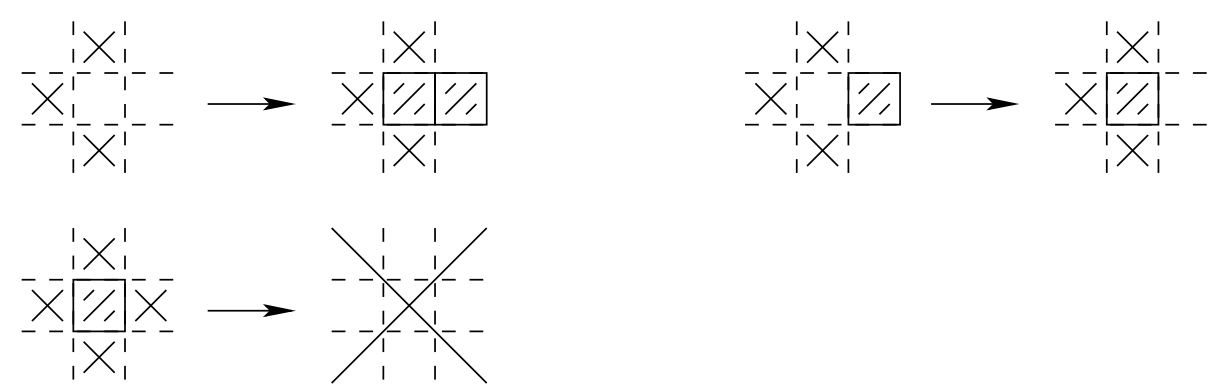

(b) Ação do operador $V_{\mathbf{r}, \boldsymbol{\delta}}^{(2)}$.
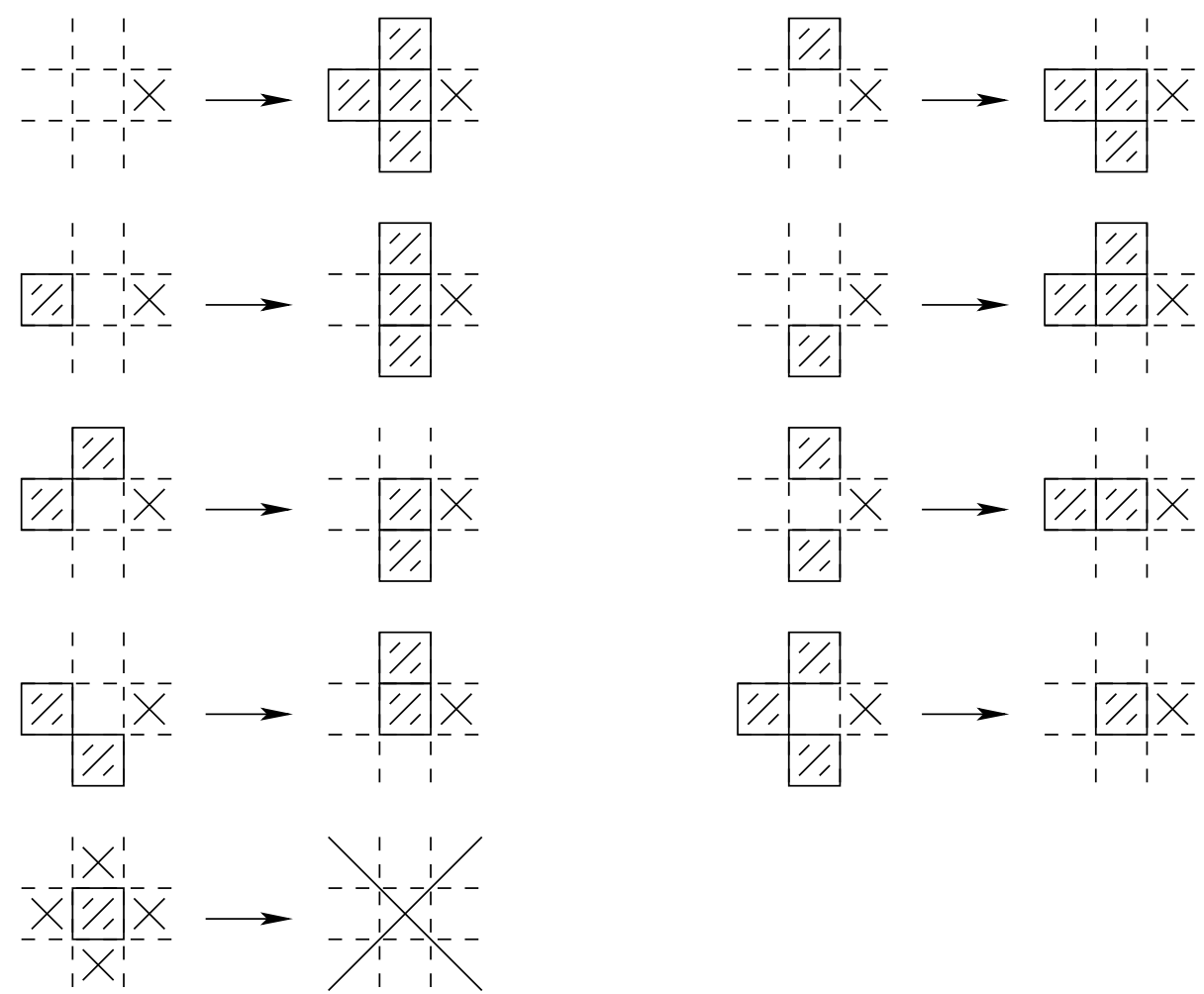

(c) Ação do operador $V_{\mathbf{r}, \boldsymbol{\delta}}^{(4)}$.

Figura 4.3: Operadores $S_{\mathbf{r}}, V_{\mathbf{r}, \boldsymbol{\delta}}^{(2)}$ e $V_{\mathbf{r}, \boldsymbol{\delta}}^{(4)}$ aplicados a uma configuração $|\eta\rangle$. O sítio $\mathbf{r}$ é o sítio central de cada diagrama, e o sítio $\mathbf{r}+\boldsymbol{\delta}$ é o sítio à direita do sítio central. Sítios marcados com $\times$ podem ter qualquer valor. Configurações marcadas com $\times$ são aniquiladas. 
como feito para o modelo de Glauber linear. Primeiramente, notamos que, para o cálculo do termo de $C_{\mathbf{0}}(t)$ que multiplica $t^{l}$, é necessário aplicar uma sequência de $l$ operadores $W_{0}, V^{(2)}$ ou $V^{(4)}$ ao vetor inicial $\left|1_{\mathbf{0}}\right\rangle$. Para que tal sequência contribua com um resultado não-nulo para o cálculo, após $l$ aplicações, o vetor obtido deve ser novamente $\left|1_{\mathbf{0}}\right\rangle$, devido ao produto escalar $\operatorname{com}\left\langle 1_{\mathbf{0}}\right|$.

Analisando a ação dos operadores $W_{0}, V^{(2)}$ e $V^{(4)}$ sobre uma configuração $|\eta\rangle$ (figura 4.3), vemos que:

- $W_{0}$ aniquila a configuração quando age em um sítio vazio, e não a altera quando age num sítio ocupado;

- $V^{(2)}$ pode criar duas partículas (quando o sítio localizado em $\mathbf{r}+\boldsymbol{\delta}$ está vazio) ou transferir a partícula do sítio $\mathbf{r}+\boldsymbol{\delta}$ para o sítio $\mathbf{r}$;

- $V^{(4)}$ pode criar 2 ou 4 partículas, transferir uma partícula para um sítio vizinho à sua posição original ou destruir um par de partículas.

Como o número de partículas pode aumentar e diminuir conforme os operadores são aplicados a uma dada configuração, é preciso considerar, enquanto fazemos os cálculos necessários para obter o termo $c_{l}$, vetores com mais de 1 partícula. Assim, este problema é mais complexo que o problema examinado nas seções 4.2 e 4.3, nos quais todas as configurações necessárias para o cálculo da susceptibilidade possuíam 2 partículas.

Devido a este aumento da complexidade do problema atual relativo aos problemas dos capítulos anteriores, não é possível obter uma solução analítica para a função autocorrelação do modelo de Glauber em duas dimensões, utilizando as técnicas exploradas nos capítulos anteriores. Para este problema, vamos obter os coeficientes $c_{l}$ numericamente, aplicando os operadores $W_{0}, V^{(2)}, V^{(4)}$ à configuração inicial e coletando o máximo de termos possível, para depois fazer uma análise da série obtida.

\subsubsection{Construção numérica da série. Resultados}

A construção numérica do termo $c_{l}$ da expansão em série envolve descobrir todas as sequências de operadores $W_{0}, V^{(2)}$ e $V^{(4)}$ de comprimento $l$ que, quando aplicadas ao vetor $\left|1_{\mathbf{0}}\right\rangle$, resultam novamente no vetor $\left|1_{\mathbf{0}}\right\rangle$. Relembrando que $W_{0}, V^{(2)}$ e $V^{(4)}$ são de fato compostos por um número infinito de operadores (um para cada sítio 
da rede para $W_{0}$, e quatro para cada sítio para $V^{(2)}$ e $V^{(4)}$ ), precisamos de uma estratégia para diminuir o número de sequências de operadores a serem analisadas. Para criar essa estratégia, impomos algumas condições que devem ser satisfeitas por uma configuração $\eta$ para que seja possível criar a configuração $\left|1_{0}\right\rangle$ após um certo número $n$ de passos.

Primeiramente, definimos a função diâmetro do aglomerado de partículas $\varphi(\eta)$ como a distância máxima entre partículas da rede (definida como o comprimento do menor caminho sobre a rede ligando os dois sítios). Em particular, a função diâmetro é nula para qualquer configuração com uma única partícula.

Para uma configuração com apenas 3 partículas, dispostas como na figura 4.4b, é possível diminuir $\varphi(\eta)$ de 2 para 0 em um passo. Para quaisquer outras configurações, $\varphi(\eta)$ diminui, no máximo, por 1. Assim, obtemos a primeira condição:

- Para que uma configuração $|\eta\rangle$ contribua para o cálculo do termo $c_{l}, \varphi(\eta)$ deve ser no máximo igual a $l-n+1$, onde $n$ é o número de operadores utilizados para criar $|\eta\rangle$.

Uma condição relacionada à primeira refere-se à distância máxima entre uma partícula e a origem, ou a função raio da configuração $\mathcal{R}(\eta)$. Um valor notável é $\mathcal{R}\left(\left|1_{0}\right\rangle\right)=0$. Diferentemente de $\varphi(\eta), \mathcal{R}(\eta)$ diminui no máximo em 1 unidade, para qualquer configuração da rede. uma vez que $\varphi(\eta)$ envolve distâncias entre partículas, e $\mathcal{R}(\eta)$ envolve distâncias entre uma partícula e um ponto fixo (ver figura 4.4 para um exemplo). A segunda condição é:

- Para que uma configuração $|\eta\rangle$ contribua para o cálculo de $c_{l}, \mathcal{R}(\eta)$ deve ser no máximo igual a $l-n$, onde $n$ é o número de operadores utilizados para criar $|\eta\rangle$.

Podemos ainda especificar uma terceira condição, envolvendo o número total de partículas na configuração:

- Uma configuração $|\eta\rangle$ contribui para o cálculo de $c_{l}$ se o número total de partículas em $\eta$ é igual a $2(l-n)+1$, onde $n$ é o número de operadores utilizados para criar $|\eta\rangle$.

Esta regra decorre do fato de o número de partículas poder diminuir no máximo em 2 unidades em cada passo. 

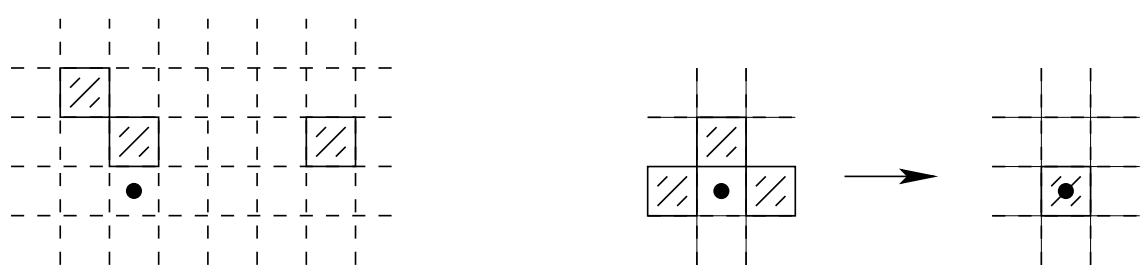

Figura 4.4: (a) Uma configuração $\eta$ com três partículas, nas posições $(4,1),(0,1)$ e $(-1,2)$. O diâmetro dessa configuração é $\varphi=6$ (distânica entre a $1^{\mathrm{a}}$ e a $3^{\mathrm{a}}$ partícula), e o raio é $\mathcal{R}=5$ (distância entre a $1^{\mathrm{a}}$ partícula e a origem). (b) Nessa transição, $\varphi$ cai de 2 para 0 , enquanto $\mathcal{R}$ cai de 1 para 0 .

Escrevemos um programa em $\mathrm{C}$ que calcula os termos $c_{l}$ da expansão em série de $C_{\mathbf{0}}(t)$ criando todas as configurações intermediárias que satisfazem às 3 regras enunciadas acima, reduzindo-as a uma forma padrão (para evitar o desperdício de memória que ocorreria ao guardar rotações e reflexões de um mesmo estado), associando a cada configuração o coeficiente numérico adequado e somando os coeficientes de todas as configurações repetidas. Entretanto, mesmo implantando as regras acima, o número de configurações que é preciso armazenar nos passos intermediários necessários para descobrir o coeficiente $c_{l}$ cresce exponencialmente com $l$ - o programa é ineficiente, no sentido em que muitas configurações intermediárias que não contribuem para o resultado final em uma certa ordem $l$ não são retiradas da memória do programa, por falta de critérios que misturem as 3 condições básicas acima.

Utilizando $A=\frac{5}{3 \sqrt{2}}$ e $B=-\frac{1}{3 \sqrt{2}}$, que correspondem ao modelo de Glauber para $\mu=\tanh 2 K_{c}=\frac{1}{\sqrt{2}}$ (ponto crítico), conseguimos obter, utilizando uma máquina com processador Athlon $643000+$ e 512 MB de memória RAM, os nove primeiros termos da série, que aparecem na tabela 4.4. Para obter o último coeficiente dessa tabela, o número máximo de configurações que foi necessário armazenar em um certo passo foi igual a 21758 configurações distintas (excluindo dessa contagem configurações que podem ser obtidas de outras por rotações e reflexões), correspondentes a sequências de 3 operadores $W_{0}, V_{2}$ ou $V_{4}$ aplicados a $\left|1_{\mathbf{0}}\right\rangle$.

Para obter o expoente crítico a partir dos termos da tabela 4.4, realizamos uma análise de Padé da série

$$
\frac{d}{d t} \ln C_{\mathbf{0}}(t)=d_{l} t^{l}
$$

Como dito anteriormente, a autocorrelação temporal deve decair de acordo com uma lei de potência no ponto crítico e exponencialmente fora do ponto crítico, de 
Tabela 4.4: Primeiros coeficientes da expansão em série de $C_{\mathbf{0}}(t)$, obtidos por contagem numérica de configurações.

\begin{tabular}{|l|r|}
\hline$l$ & \multicolumn{1}{|c|}{$c_{l}$} \\
\hline 0 & $1,0000000000 \mathrm{E}+00$ \\
1 & $-1,0000000000 \mathrm{E}+00$ \\
2 & $6,7361111111 \mathrm{E}-01$ \\
3 & $-3,4027777778 \mathrm{E}-01$ \\
4 & $1,2703350052 \mathrm{E}-01$ \\
5 & $-2,4485596708 \mathrm{E}-01$ \\
6 & $-1,1071013126 \mathrm{E}-02$ \\
7 & $1,5590124962 \mathrm{E}-02$ \\
8 & $-1,0029441538 \mathrm{E}-02$ \\
\hline
\end{tabular}

acordo com (4.160). Assim, a série correspondente a $\frac{d}{d t} \ln C_{\mathbf{0}}(t)$ deve se comportar como:

$$
\frac{d}{d t} \ln C_{\mathbf{0}}(t)= \begin{cases}-1 / \tau, & \text { fora do ponto crítico } \\ -\nu_{\|} / t, & \text { no ponto crítico }\end{cases}
$$

Ou seja, é esperado que, no ponto crítico, esta série apresente um polo real para $t_{c}=0$ e seu resíduo deve ser igual ao expoente crítico $\nu$ procurado. Uma análise de Padé da série $\frac{d}{d t} \ln C_{\mathbf{0}}(t)$ deve mostrar o polo se aproximando da origem conforme o número de termos da série original aumenta, enquanto o resíduo correspondente corresponde a uma estimativa do expoente crítico.

A partir dos dados da tabela 4.4, é possível construir aproximantes de Padé da série $\frac{d}{d t} \ln C_{\mathbf{0}}(t)$ de ordem $[N, N]$, com $N \leq 3$. Os termos da série $\frac{d}{d t} \ln C_{0}(t)$ aparecem na tabela 4.5, enquanto as aproximações obtidas para o polo e o resíduo desta série por aproximantes de Padé de ordem $[N, N]$ aparecem na tabela 4.6.

Analisando os resultados da tabela 4.6, não é possível notar nenhuma convergência nos valores do polo e do expoente $\nu_{\|}$conforme $N$ varia e trabalhamos com mais termos da tabela 4.4. Concluímos que o número de termos obtidos numericamente não é o suficiente para que possamos obter uma estimativa confiável do expoente crítico, uma vez que não é possível observar uma tendência para a localização do polo após ter calculado apenas 3 aproximantes de Padé. É necessário calcular um número muito maior de termos da série de $C_{0}(t)$ para obter o expoente crítico $\nu$ com uma precisão adequada e, para chegar a este resultado utilizando a técnica exposta nesta seção, é necessário obter as configurações de cada ordem da 
Tabela 4.5: Primeiros coeficientes $d_{l}$ da série $\frac{d}{d t} \ln C_{0}(t)^{1}$.

\begin{tabular}{|c|c|}
\hline$l$ & $d_{l}$ \\
\hline 0 & $-1,0000000000$ \\
1 & 0,3472222222 \\
2 & $-7,00110^{-13}$ \\
3 & $-0,0660365226$ \\
4 & $-1,0451308513$ \\
5 & $-1,3560388161$ \\
6 & $-0,4914185287$ \\
7 & $-0,6771854800$ \\
\hline
\end{tabular}

Tabela 4.6: Polo e expoente crítico $\nu$ obtidos a partir da aproximação de Padé de ordem $[N, N]$ dos coeficientes da tabela 4.5.

\begin{tabular}{|c|c|c|}
\hline$N$ & $t_{c}$ & $\nu$ \\
\hline 1 & $-2,8800000000$ & 2,8800000000 \\
2 & 0,0632329810 & $1,056548 \mathrm{E}-06$ \\
3 & -1.13382 & 0,2222424378 \\
\hline
\end{tabular}

expressão (4.173) de modo mais eficiente, descobrindo quais configurações obtidas a cada ordem podem ser descartadas sem afetar o resultado final. Deste modo, a análise do modelo GG também deve ser paralizada no momento, podendo ser continuada no futuro, quando for possível obter um número maior de termos da sériede $C_{0}(t)$.

\footnotetext{
${ }^{1}$ Provavelemente, o coeficiente $d_{2}$ é exatamente nulo
} 


\section{Capítulo 5}

\section{Considerações finais}

O estudo de modelos estocásticos fora do equilíbrio é uma área em desenvolvimento, com muito potencial para novas descobertas e expansão de conceitos. Em particular, a investigação de modelos envolvendo simultaneamente diversas simetrias e mecanismos de evolução temporal, como foi feito nesta tese, pode levar a um entendimento ainda maior dos fatores que caracterizam a evolução de sistemas físicos que interagem com o universo ao redor.

Nesta tese, foram apresentados três modelos estocásticos. No capítulo 2, foi introduzido brevemente o processo de contato, que é um dos modelos básicos da Física Estatística fora do equilíbrio, devido a ser um dos modelos com regras mais simples que apresenta uma transição de fase em uma dimensão, entre um estado ativo, para taxas de aniquilação baixas, e uma configuração absorvente (vácuo), que ocorre quando a taxa de aniquilação aumenta além de um valor crítico. Este modelo foi estudado por meio de uma expansão em série das probabilidades de aglomerados de $n$ sítios em função da taxa de aniquilação $\alpha$, e foi feita uma análise de Padé dos coeficientes do parâmetro de ordem, de modo a determinar a taxa crítica e o expoente crítico correspondente, obtendo-se resultados consistentes com os obtidos em outras referências.

A seguir, no capítulo 3, foi apresentado um modelo de pilha de areia relacionado ao modelo de pilha de areia com altura restrita com regra de transição independente, apresentado por Dickman, Tomé e Oliveira [12]. A relevância de se estudar modelos como este reside, entre outro fatores, na relação íntima entre modelos com uma transição de fase para muitos possíveis estados absorventes e um campo conservado pela dinâmica do modelo e o fenômeno de criticalidade auto-organizada, apontado 
por muitos como uma das possíveis causas para a existência de muitos fenômenos físicos regidos por leis de potência. Por outro lado, dentro do contexto da Física Estatística fora do equilíbrio, o modelo de pilha de areia com densidade constante parece ser um dos primeiros representantes de uma nova classe de universalidade, devido a apresentar um conjunto de expoentes críticos diferentes, por exemplo, da classe DP (percolação direcionada). Na falta de uma teoria fundamental que unifique o estudo dos modelos estocásticos fora do equilíbrio, estudar e categorizar as diferentes classes de universalidade existentes pode ser uma alternativa consistente.

Um dos fatores básicos para a caracterização do modelo estudado nesta tese é a introdução da assimetria espacial no mecanismo de evolução temporal, levando a um processo de difusão preferencial em um sentido. A simulação computacional e as aproximações de campo médio mostram que, para valores extremos do parâmetro de assimetria $p$ do problema, o modelo comporta-se de forma qualitativemente diferente: a existência de mais campos conservados (a densidade de sítios ativos) implica a inexistência de uma transição para uma fase absorvente.

Para os demais valores de $p$, os expoentes críticos que caracterizam a transição para a fase absorvente são os mesmos para qualquer valor de $p$, exceto quando o modelo deixa de ser assimétrico (em $p=0,5$ ), quando eles assumem os valores obtidos por Dickman [18]. Concluímos que a assimetria introduzida no sistema é um fator relevante para mudar a classe de universalidade do modelo estudado. Também foi estudado, tanto por aproximações de campo médio quanto por simulação, o fluxo de partículas resultante da assimetria nas regras de evolução.

A seguir, no capítulo 4, foi apresentado um modelo intimamente relacionado ao modelo de Glauber, proposto pela primeira vez em 1963 por R. Glauber como um modelo dinâmico cujo estado estacionário reproduz a probabilidade de Gibbs de um sistema de Ising. Embora este modelo já seja bastante conhecido e estudado, ele ainda pode servir como ponto de partida para desenvolvimentos novos e interessantes, no sentido de ampliar nosso conhecimento atual sobre as propriedades estáticas e dinâmicas de modelos estocásticas.

Primeiramente, trabalhamos com o modelo de Glauber linear, cuja taxa de transição de spin reproduz a do modelo de Glauber em 1 dimensão, e calculamos sua susceptibilidade magnética a campo nulo utilizando o método de expansão em série perturbativa. O resultado obtido é equivalente ao obtido por outros métodos. Mostramos a seguir que é possível resolver o mesmo problema para um número qualquer de dimensões utilizando o método de expansão em série. Notamos que, para 
$d \neq 1$, o modelo de Glauber linear deixa de reproduzir o modelo de Glauber, exceto no ponto $\lambda=0$, que corresponde ao limite de temperatura infinita no modelo de Glauber. Os resultados obtidos para o expoente crítico novamente são iguais aos obtidos por outros métodos; entretanto, durante os cálculos, encontramos identidades novas e demonstramos como um poderoso algoritmo para a manipulação de números binomiais e termos hipergeométricos em geral pode ser útil para a manipulação de somas em Mecânica Estatística e no estudo de processos estocásticos.

Finalmente, a partir da relação entre o modelo de Glauber e o modelo de Glauber linear em 2 dimensões, definimos um modelo dependente de 2 parâmetros $A$ e $B$, que engloba os dois modelos citados para valores especiais de $A$ e $B$, e mostramos como estudar todo o espaço de fase deste modelo por meio de um método de contagem numérica de configurações e análise de Padé dos coeficientes da série. Entretanto, ao tentar calcular o expoente crítico $\nu_{\|}$para o modelo de Glauber em duas dimensões, verificamos que, devido ao aumento exponencial do número de configurações que deve ser armazenado na memória do programa, não pudemos avançar muito na ordem $l$ do cálculo. À medida que mais memória se torne disponível, esta estratégia de pesquisa pode ser retomada. 



\section{Apêndice A}

\section{Algumas expansões em série}

Nesta seção, vamos demonstrar os resultados utilizados na última linha da equação (4.76) e na equação (4.78):

$$
\begin{aligned}
(1-4 z)^{-1 / 2} & =\sum_{n=0}^{\infty}\left(\begin{array}{c}
2 n \\
n
\end{array}\right) z^{n} \\
(1-4 z)^{1 / 2} & =1-\sum_{n=1}^{\infty} \frac{1}{2 n-1}\left(\begin{array}{c}
2 n \\
n
\end{array}\right) z^{n}
\end{aligned}
$$

Para obter o resultado (A.1), começamos expandindo o binômio do lado esquerdo da igualdade em torno de $z=0$ :

$$
K(z)=1-\left(\frac{-1}{2}\right) 4 z+\left(\frac{-1}{2}\right)\left(\frac{-3}{2}\right) \frac{(4 z)^{2}}{2 !}-\left(\frac{-1}{2}\right)\left(\frac{-3}{2}\right)\left(\frac{-5}{2}\right) \frac{(4 z)^{3}}{3 !}+\ldots
$$

Notamos que todos os termos são positivos e que os coeficientes $c_{n}$ de $(4 z)^{n} / n$ ! podem ser escritos como:

$$
\begin{aligned}
c_{0} & =1 \\
c_{n} & =\frac{(2 n-1) ! !}{2^{n}}, \quad n \geq 1
\end{aligned}
$$

onde $k ! !=k(k-2) !$ ! e define-se $1 ! !=0 ! !=1$. Podemos reescrever o fatorial duplo de um número ímpar $(2 n-1)$ !! usando a identidade:

$$
(2 n-1) ! !(2 n) ! !=(2 n) ! \rightarrow(2 n-1) ! !=\frac{(2 n) !}{(2 n) ! !}=\frac{(2 n) !}{2^{n} n !}
$$


Substituindo (A.5) em (A.4), obtemos:

$$
K(z)=1+\sum_{n=1}^{\infty} \frac{(2 n) !}{2^{n} n !} \frac{(4 z)^{n}}{2^{n} n !}=1+\sum_{n=1}^{\infty} \frac{(2 n) !}{(n !)^{2}} z^{n}=\sum_{n=0}^{\infty}\left(\begin{array}{c}
2 n \\
n
\end{array}\right) z^{n}
$$

Para a expansão em série de (A.2), adotamos o mesmo procedimento:

$$
\begin{aligned}
H(z) & =(1-4 z)^{1 / 2} \\
& =1-\left(\frac{1}{2}\right) 4 z+\left(\frac{1}{2}\right)\left(\frac{-1}{2}\right) \frac{(4 z)^{2}}{2 !}-\left(\frac{1}{2}\right)\left(\frac{-1}{2}\right)\left(\frac{-3}{2}\right) \frac{(4 z)^{3}}{3 !}+\ldots \\
& =1-\sum_{n=1}^{\infty} \frac{(2 n-1) ! !}{2 n-1} \frac{(4 z)^{n}}{2^{n} n !} \\
& =1-\sum_{n=1}^{\infty} \frac{(2 n) !}{2^{n} n !(2 n-1)} \frac{(4 z)^{n}}{2^{n} n !} \\
& =1-\sum_{n=1}^{\infty} \frac{1}{2 n-1}\left(\begin{array}{c}
2 n \\
n
\end{array}\right) z^{n}
\end{aligned}
$$




\section{Apêndice B}

\section{O algoritmo de Gosper-Zeilberger}

Nesta seção, mostraremos como a identidade (4.147) pode ser demonstrada, utilizando o algoritmo de Gosper-Zeilberger como exposto por Knuth, Pathsnik e Graham [30, 34, 35].

Seja um "termo próprio" $t(n, k)$ da forma:

$$
t(n, k)=f(n, k) \frac{\left(a_{1} n+a_{1}^{\prime} k+a_{1}^{\prime \prime}\right) ! \ldots\left(a_{p} n+a_{p}^{\prime} k+a_{p}^{\prime \prime}\right) !}{\left(b_{1} n+b_{1}^{\prime} k+b_{1}^{\prime \prime}\right) ! \ldots\left(b_{q} n+b_{q}^{\prime} k+b_{q}^{\prime \prime}\right) !} w^{n} z^{k}
$$

onde $f(n, k)$ é um polinômio em $n$ e $k, w, z \neq 0, a_{1}, a_{1}^{\prime}, \ldots a_{p}, a_{p}^{\prime}, b_{1}, b_{1}^{\prime} \ldots b_{q}, b_{q}^{\prime}$ são constantes inteiras e os demais coeficientes são números complexos arbitrários. Então, existem polinômios $\beta_{0}(n), \ldots \beta_{l}(n)$ e um termo próprio $T(n, k)$ tais que:

$$
\beta_{0}(n) t(n, k)+\cdots+\beta_{l}(n) t(n+l, k)=T(n, k+1)-T(n, k)
$$

A soma no índice $k$ do termo próprio $t(n, k)$ pode ser obtida da expressão acima, como uma relação de recorrência no índice $n$. Seja $S_{n}=\sum_{k=0}^{\infty} t(n, k)$ : podemos obter uma relação para $S_{n}$ somando (B.2) no índice $k$, obtendo:

$$
\beta_{0}(n) S_{n}+\cdots+\beta_{l}(n) S_{n+l}=\lim _{k \rightarrow \infty} T(n, k)-T(n, 0)
$$

O algoritmo de Gosper-Zeilberger fornece instruções para encontrar os polinômios $\beta_{0}(n), \ldots \beta_{l}(n)$ e o termo próprio $T(n, k)$, de modo que, conhecendo-se o termo $t(n, k)$, possamos obter a somatória $S_{n}$ diretamente (quando $l=0$ ) ou por meio de uma relação de recorrência. 
Para a demonstração da identidade (4.147), tomemos:

$$
t(n, k)=\frac{(2 n) !}{(k !)^{2}[(n-k) !]^{2}}
$$

Vemos que $t(n, k)$ corresponde a um termo próprio, tomando a definição (B.1) e fazendo as substituições $f(n, k)=1, p=1, q=4, a_{1}=2, b_{1}^{\prime}=b_{2}^{\prime}=b_{3}=b_{4}=1$, $b_{3}^{\prime}=b_{4}^{\prime}=-1, w=z=1$, com todas as demais constantes nulas.

Para aplicar o algoritmo de Gosper-Zeilberger, primeiramente escolhemos o número de polinômios $\beta_{l}(n)$ necessário para que (B.2) possa ser satisfeita. Tomando $l=0$, o algoritmo falha: são necessários dois polinômios $\beta_{l}(n)$ para a demonstração, os quais vamos denominar $\beta_{0}(n)$ e $\beta_{1}(n)$.

Vamos definir $\hat{t}(n, k)$ como o lado esquerdo da igualdade (B.2):

$$
\hat{t}(n, k)=\beta_{0}(n) t(n, k)+\beta_{1}(n) t(n+1, k)
$$

Reescrevendo $t(n+1, k)$ em função de $t(n, k)$ na igualdade acima, podemos escrever $\hat{t}(n, k)$ como o produto de um polinômio $p(n, k)=\sum_{i=0}^{l} \gamma(n, k) \beta_{i}(n)$ por uma função $\bar{t}(n, k)$ que dependa apenas de $t(n, k)$. Obtemos:

$$
\begin{aligned}
\bar{t}(n, k) & =\frac{t(n, k)}{(n+1-k)^{2}} \\
p(n, k) & =(n+1-k)^{2} \beta_{0}(n)+(2 n+2)(2 n+1) \beta_{1}(n)
\end{aligned}
$$

Pelo algoritmo de Gosper-Zeilberger, procuramos polinômios $\hat{p}(n, k), q(n, k)$ e $r(n, k)$ que satisfaçam à igualdade:

$$
\frac{\hat{t}(n, k+1)}{\hat{t}(n, k)}=\frac{\hat{p}(n, k+1)}{\hat{p}(n, k)} \frac{q(n, k)}{r(n, k+1)}
$$

Segundo a referência [30], na maioria das situações em que o algoritmo é utilizado, a escolha $\hat{p}(n, k)=p(n, k)$ é adequada. Com essa esolha, os polinômios $q(n, k)$ e $r(n, k)$ são dados por:

$$
\begin{aligned}
& q(n, k)=(n+1-k)^{2}=k^{2}-2(n+1) k+(n+1)^{2} \mathrm{e} \\
& r(n, k)=k^{2}
\end{aligned}
$$


Procuramos agora um polinômio $s(n, k)$ em $k$ que satisfaça à igualdade:

$$
\hat{p}(n, k)=q(n, k) s(n, k+1)-r(n, k) s(n, k)
$$

Conhecendo $s(n, k)$, podemos escrever o termo próprio $T(n, k)$ como:

$$
T(n, k)=r(n, k) s(n, k) \bar{t}(n, k)
$$

Para encontrar $s(n, k)$, escrevemos esse polinômio na forma geral:

$$
s(n, k)=\alpha_{0}(n)+\alpha_{1}(n) k+\ldots \alpha_{d}(n) k^{d}
$$

Para determinar os termos $\alpha_{0}, \ldots \alpha_{d}$, igualamos os coeficientes das diferentes potências de $k$ nos dois lados de (B.11). Antes, o grau $d$ de $s(n, k)$ deve ser determinado. Aplicando o método exposto em [30], descobrimos que $d=1$, de modo que:

$$
p(n, k)=q(n, k)\left\{\alpha_{0}(n)+\alpha_{1}(n)(k+1)\right\}-r(n, k)\left\{\alpha_{0}(n)+\alpha_{1}(n) k\right\}
$$

Substituindo (B.7), (B.9) e (B.10) em (B.14), obtemos 3 equações lineares homogêneas nas 4 incógnitas $\alpha_{0}(n), \alpha_{1}(n), \beta_{0}(n)$ e $\beta_{1}(n)$. Este sistema possui uma família de soluções:

$$
\begin{aligned}
\alpha_{0}(n) & =-\frac{3}{2}(n+1) \alpha, \\
\alpha_{1}(n) & =\alpha, \\
\beta_{0}(n) & =-(2 n+1) \alpha, \\
\beta_{1}(n) & =\frac{(n+1)^{2}}{4(2 n+1)} \alpha
\end{aligned}
$$

Conhecendo o polinômio $s(n, k)$, podemos construir $T(n, k)$ :

$$
T(n, k)=r(n, k) s(n, k) \bar{t}(n, k)=\alpha k^{2}\left[k-\frac{3}{2}(n+1)\right] \frac{t(n, k)}{(n+1-k)^{2}}
$$

Agora, podemos escrever uma relação de recorrência para $S_{n}=\sum_{k=0}^{n} t(n, k)$. Substituindo (B.17), (B.18) e (B.19) em (B.3), obtemos:

$$
-(2 n+1) S_{n}+\frac{(n+1)^{2}}{4(2 n+1)} S_{n+1}=\lim _{k \rightarrow \infty}\{T(n, k+1)\}-T(n, 0)=0
$$


Vemos facilmente que $S_{0}=t(0,0)=1$. Reescrevemos a relação acima substituindo $n$ por $n-1$, obtendo:

$$
\begin{aligned}
S_{0} & =1 \\
S_{n} & =\frac{4(2 n-1)^{2}}{n^{2}} S_{n-1}, \quad n \geq 1
\end{aligned}
$$

de onde obtemos a forma geral de $S_{n}$ :

$$
S_{n}=\sum_{k=0}^{n} \frac{(2 n) !}{(k !)^{2}[(n-k) !]^{2}}=4^{n}\left[\frac{(2 n-1) ! !}{n !}\right]^{2}=4^{n}\left[\frac{(2 n) !}{2^{n} n ! n !}\right]^{2}=\left(\begin{array}{c}
2 n \\
n
\end{array}\right)^{2}
$$




\section{Referências Bibliográficas}

[1] S. R. A. Salinas, Introdução à Física Estatística, Edusp, São Paulo (1999).

[2] K. Huang, Statistical Mechanics, John Wiley \& Sons, Inc. (1963).

[3] G. Ódor, Rev. Mod. Phys. 76, 663 (2004);

[4] H. Hinrichsen, Adv. Phys. 49, 815 (2000);

[5] T. Tomé, M. J. de Oliveira, Dinâmica Estocástica e Irreversibilidade, Edusp, São Paulo (2001);

[6] T. E. Harris, Ann. Probab. 2, 969 (1974);

[7] I. Jensen e R. Dickman, J. Stat. Phys 71, 89 (1993);

[8] P. Bak, C. Tang e K. Wiesenfeld, Phys. Rev. Lett. 59, 381(1987); Phys. Rev. A 38, 364 (1988);

[9] D. Dhar, Physics A 263, 4 (1999);

[10] S. S. Manna, J. Phys A 24, L363 (1991);

[11] A. Vespignani e S. Zapperi, Phys. Rev. Lett. 78, 4793 (1997); Phys. Rev. E. $\mathbf{5 7 ,} 6345$ (1998);

[12] R. Dickman, T. Tomé e M. J. de Oliveira, Phys. Rev E 66, 016111 (2002);

[13] E. F. Silva (dissertação de mestrado), M. J. de Oliveira, J. Phys. A 41, 385004 (2008);

[14] K. Binder e D. W. Heermann, Monte Carlo Simulation in Statistical Physics, Springer-Verlag, Berlim (1992); 
[15] M. E. J. Newman e G. T. Barkena, Monte Carlo Methods in Statistical Physics, Oxford University Press (1999);

[16] J. M. Yeomans, Statistical Mechanics of Phase Transitions, Oxford University Press, 1992;

[17] J. L. Cardy (ed.) Finite-size Scaling (Current Physics - Sources and Comments, vol. 2, North-Holland (1988);

[18] R. Dickman, Phys. Rev. E 73, 036131 (2006);

[19] M. J. de Oliveira, Phys. Rev E 67, 066101 (2003);

[20] R. J. Glauber, J. Math. Phys 4, 294 (1963);

[21] E. Ising, Z. Physik 31, 253 (1925);

[22] L. Onsager, Phys. Rev. 65, 117 (1944);

[23] C. Domb e M. S. Green (eds.), Phase Transitions and Critical Phenomena, vol. 3, Academic Press (1974);

[24] R. Dickman, A. Vespignani e S. Zapperi, Phys. Rev. E. 57, 5095 (1998);

[25] W. G. Dantas e J. F. Stilck, Braz. J. Phys. 36, 750 (2006);

[26] R. L. Burden e J. D. Faires, Análise Numérica, Cengage Learning, 8ª edição, 2008;

[27] K. Binder, Phys. Rev. Lett. 47, 693 (1981);

[28] T. Tomé e M. J. de Oliveira, Phis. Rev. E 72, 026130 (2005);

[29] R. Dickman e J. Kamphorst Leal da Silva, Phys. Rev. E 58, 4266 (1998);

[30] D. E. Knuth, O. Patashnik, R. L. Grahan, Matemática Concreta - Fundamentos para a Ciência da Computação, Ed. LTC, Rio de Janeiro, 1995;

[31] I. S. Gradshteyn e I. M. Ryzhik, Table of integrals, series and products, Academic Press, Inc., 7th ed., 2007;

[32] T. Morita e T. Horiguchi, J. Math. Phys. 12, 986 (1971); 
[33] G. Arfken, Mathematical Methods for Physicists, Academic Press, Inc., 1985;

[34] R. W. Gosper, Jr., Proc. Natl. Acad. Sci. USA 75, 40 (1978);

[35] D. Zeilberger, J. Symb. Comput. 11, 195 (1991). 